\title{
Frail elderly : identification and disability prevention in primary care
}

Citation for published version (APA):

Daniëls, R. (2011). Frail elderly : identification and disability prevention in primary care. [Doctoral Thesis, Maastricht University]. Datawyse / Universitaire Pers Maastricht. https://doi.org/10.26481/dis.20110707rd

Document status and date:

Published: 01/01/2011

DOI:

10.26481/dis.20110707rd

Document Version:

Publisher's PDF, also known as Version of record

\section{Please check the document version of this publication:}

- A submitted manuscript is the version of the article upon submission and before peer-review. There can be important differences between the submitted version and the official published version of record.

People interested in the research are advised to contact the author for the final version of the publication, or visit the DOI to the publisher's website.

- The final author version and the galley proof are versions of the publication after peer review.

- The final published version features the final layout of the paper including the volume, issue and page numbers.

Link to publication

\footnotetext{
General rights rights.

- You may freely distribute the URL identifying the publication in the public portal. please follow below link for the End User Agreement:

www.umlib.nl/taverne-license

Take down policy

If you believe that this document breaches copyright please contact us at:

repository@maastrichtuniversity.nl

providing details and we will investigate your claim.
}

Copyright and moral rights for the publications made accessible in the public portal are retained by the authors and/or other copyright owners and it is a condition of accessing publications that users recognise and abide by the legal requirements associated with these

- Users may download and print one copy of any publication from the public portal for the purpose of private study or research.

- You may not further distribute the material or use it for any profit-making activity or commercial gain

If the publication is distributed under the terms of Article $25 \mathrm{fa}$ of the Dutch Copyright Act, indicated by the "Taverne" license above, 


\section{Frail elderly}

Identification and disability prevention

in primary care 
CAPHRI School for Public Health and Primary Care Department of Health Care and Nursing Science Faculty of Health, Medicine and Life Science Maastricht University

Maastricht, the Netherlands

Centre of Research on Autonomy and Participation Faculty of Health and Care

Zuyd University of Applied Sciences

Heerlen, the Netherlands

ISBN 9789461590688

(C) Copyright R Daniëls, Heerlen, Juni 2011

E-mail: r.daniels@hszuyd.nl

Productie: Datawyse / Universitaire Pers Maastricht Cover illustration: www.Flickr.com 


\title{
Frail elderly
}

\section{Identification and disability prevention in primary care}

\author{
PROEFSCHRIFT \\ ter verkrijging van de graad van doctor aan de Universiteit Maastricht, \\ op gezag van de Rector Magnificus, Prof. mr. G.P.M.F. Mols, \\ volgens het besluit van het college van Decanen, \\ in het openbaar te verdedigen, \\ op donderdag 7 juli 2011 om 10:00 uur
}

door

Ramon Daniëls

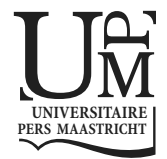




\section{Promoteres}

Prof. dr. WJA van den Heuvel

Prof. dr. LP de Witte

\section{Copromotor}

Dr. E van Rossum

\section{Beoordelingscommissie}

Prof. dr. JMGA Schols (voorzitter)

Prof. dr. RA de Bie

Dr. MJL Graff (Radboud University)

Prof. dr. MJ Schuurmans (Utrecht University)

Prof. dr. GDEM van der Weijden 
The happiest people spend much time in a state of flow - the state in which people are so involved in an activity that nothing else seems to matter; the experience itself is so enjoyable that people will do it even at great cost, for the sheer sake of doing it.

Mihaly Csikszentmihalyi 



\section{CONTENTS}

Chapter 1 General Introduction 9

Chapter 2 Frailty in older age: Concepts and relevance for occupational and physical therapy

Physical and Occupational Therapy in Geriatric 2008;27(2): 81-95

Chapter 3 The psychometric properties of three self-report screening instruments for identifying frail older people in the community BMC Public Health 2010;10:176

Chapter 4 The predictive validity of three self-report screening instruments for identifying frail older people in the community submitted for publication

Chapter 5 Interventions to prevent disability in frail community-dwelling elderly: a systematic review BMC Health Services Research 2008;8:278

Chapter 6 Interventions to prevent disability in frail community-dwelling older people: an overview European Journal of Ageing 2010;7:37-55

Chapter 7 A disability prevention programme for community-dwelling frail older persons

Clinical Rehabilitation. In Press

Chapter 8 Feasibility of an interdisciplinary programme to prevent disability in frail older persons

submitted for publication

Chapter 9 General Discussion

$\begin{array}{ll}\text { Summary } & 165\end{array}$

$\begin{array}{ll}\text { Samenvatting } & 171\end{array}$

$\begin{array}{lll}\text { Appendix Frailty instruments: Overview of all items } & 177\end{array}$

Steps of the disability prevention programme

Dankwoord

$\begin{array}{ll}\text { Curriculum Vitae } & 187\end{array}$

Publications 

CHAPTER 1

General Introduction 


\section{Ageing society}

The population is ageing in Western countries. In Europe, the number of elderly people is expected to almost double in the next decade. The number of oldest-old (aged 80 years and above) is estimated to triple in $2060 .^{1}$ As older people are more susceptible to disease, the demand for care will increase. ${ }^{2}$ Supporting the wellbeing of elderly becomes a challenge for European countries. In 50 years, the EU will move from having 4 working-age people for every person aged over 65 to a ratio of 2 to 1 . Overall the workforce is expected to shrink with a major impact on budgets. ${ }^{1}$ Balancing the costs and benefits of healthcare will be the key aim for ageing societies, and older people need to be supported to remain healthy and independent as long as possible. ${ }^{1}$ Therefore, a strategic shift to prevention and early intervention is necessary. ${ }^{3}$ Prevention might be most beneficial for those at high risk for dependency and disability, frail older people. ${ }^{4}$

\section{Frailty}

Frailty is a rather new concept in geriatric research and practice. Over the last decades, there has been an exponential rise in the use of the term "frailty" in the literature. ${ }^{5}$ Frail older people are at high risk for developing adverse outcomes as disability, morbidity, mortality, hospitalization and institutionalization. ${ }^{6}$ Frailty confers loss of independence and impairs the quality of life and psychological well-being of older people. It also poses challenges on families, caregivers, and other structures of social care and support. Prevalence estimates of frailty vary depending on its definition and measurement. Based on data from 10 European countries, Santos-Eggiman et al. ${ }^{7}$ estimated that $17 \%$ of older persons of 65 or older are frail. In this study, physical frailty markers ${ }^{6}$, were used to identify frail old persons. In the Netherlands, $11.3 \%$ of Dutch community-dwelling older persons ( $\geq 65$ years) would be frail according to these criteria. A study by Schuurmans et al. ${ }^{8}$, in which a broader perspective on frailty was used, identified $32 \%$ of Dutch community dwelling elderly ( $\geq 65$ years) as frail. The Ministry of Health, Welfare, and Sport ${ }^{9}$ estimates the number of frail elderly at 247.000, expected to double in 2040 to 505.000 .

There is an ongoing debate about the frailty concept. ${ }^{10}$ Agreement exists on the core feature of frailty: an increased vulnerability to stressors due to impairments in multiple, inter-related systems that lead to decline in homeostatic reserve and resiliency. ${ }^{11}$ However, there is still discussion on the definition of frailty, what criteria should be used for its recognition, and its relationships with aging, disability, and chronic disease. ${ }^{10,11}$ A review by Aminzadeh et al. ${ }^{12}$ into frailty interventions revealed a lack of consensus for an explicit conceptual definition of frailty, standard- 
ized targeting criteria, and validated practical screening criteria. The debate in literature has mainly focused on whether frailty should be defined purely in terms of biomedical factors or whether psychosocial and cognitive factors should be included as well. ${ }^{13}$

Despite a lack of consensus about the frailty definition, there seems to be agreement on the clinical usefulness of the concept. ${ }^{11}$ Whatever the inclusion criteria or the population addressed, various studies report on the increased risk for those identified as frail for adverse outcomes. ${ }^{14}$ The concept of frailty is believed to be very helpful for clinicians and researchers to understand the heterogeneity and inequalities of health trajectories with aging and to offer practitioners useful tools for patient care. ${ }^{15,16}$

\section{Disability prevention}

In early definitions, frailty was just another term for disability. ${ }^{5}$ Nowadays, frailty is considered as one of the pathways to disability, and it is assumed that prevention and interventions can be aimed at either delaying the onset of frailty or reducing disabilities. ${ }^{17}$ Disability, defined as experienced difficulty in performing activities in any domain of life, refers to several dimensions of activities: basic and instrumental activities of daily living, paid and unpaid role activities, social and leisure activities. ${ }^{18}$ For (frail) older people, difficulties in performing activities feature among the most important determinants of a reduced quality of life. ${ }^{19,20}$ Effective intervention programmes for disability prevention are needed. ${ }^{4,19}$ However, intervention research in the field of frail elderly is relatively new and so far, studies show conflicting findings and gaps in knowledge. ${ }^{12}$

In short, it is obvious that disability prevention in frail elderly is an important aim, though many questions need to be answered. How to define frailty, how to identify frail elderly, and what interventions are effective in preventing disability in frail elderly is still unclear.

\section{Dutch policy regarding frail elderly}

Similar to other Western countries, the number of Dutch elderly people increases substantially in the coming years. Currently, $14 \%$ of the Dutch population is 65 or older. This will rise to more than $20 \%$ in 2025 with a peak at about $25 \%$ in $2040 .^{9}$ The majority of elderly people are doing rather well and live independently. ${ }^{21}$ At the age of 65 , Dutch older people can expect, on average, more than 12 years without 
physical disabilities. ${ }^{21}$ Yet, some elderly are unable to be independent and participate in society.

Supporting participation and independent living are key policy targets for elderly care of the Dutch government. ${ }^{22}$ Up to 2002 , frailty was hardly 'visible' in Dutch policy reports. ${ }^{23}$ However, recent reports from agencies, such as the Dutch Health Council $^{4}$ and influential professional organizations (Royal Dutch Medical Association $^{24}$, National Association of General Practitioners ${ }^{25}$ ), put a major focus on frailty as an important determinant for adverse outcomes and need for interventions. These organizations emphasise the need for developing and evaluating tailor-made interventions that focus on promoting independent functioning in daily life for (vulnerable) older persons with an important role for primary care, screening of vulnerable groups, case management, and multidisciplinary cooperation. Investing in optimizing primary care is a strategy to control costs as it is expected to prevent institutional care and promote consistency and coordination of individual care. ${ }^{26}$

Overall the Dutch primary care system, with the GP in a key role, provides high quality care. ${ }^{27}$ Still, primary care seems insufficiently equipped to address the needs of frail elderly and prevent disability. ${ }^{4}$ The reactive approach and lack of collaboration between disciplines poses challenges. ${ }^{4,2}$ Much is expected of the coordinating role of general practitioners. A study by Schers et al. ${ }^{29}$, among 172 Dutch general practitioners, shows that the majority thinks that care of frail elderly should take place in general practice They also recognizes that this care should be improved considerably. Screening, pro-active home visits, multidisciplinary cooperation, a central role for the practice nurse, and consultation of (social) geriatricians are considered keys to improvement.

\section{OBJECTIVES AND RESEARCH QUESTIONS}

The main objective of this thesis is to develop an evidence based and feasible disability-prevention programme for community-dwelling frail elderly To achieve this, several issues related to the concept of frailty, identification of frail elderly, and the approach and organization of disability prevention need to be addressed.

- What is the state of art in the frailty literature concerning the concept of frailty and instruments for identifying frail elderly?

- What is the validity of postal screening questionnaires to identify communitydwelling frail elderly? 
- What is known in the literature about effective (elements of) disabilityprevention programmes and how can they be integrated into a comprehensive and coherent programme?

- To what extent is a new interdisciplinary disability-prevention programme for community-dwelling frail elderly feasible in Dutch primary care?

\section{Outline of this thesis}

An overview of the literature related to the concept of frailty and screening instruments for identification of community-dwelling frail elderly is presented in chapter 2. Chapter 3 reports about the internal consistency and construct validity of three frailty instruments used for postal screening to identify community-dwelling frail elderly: the Groningen Frailty Indicator, the Tilburg Frailty Indicator, and the Sherbrooke Postal Questionnaire. Chapter 4 focuses on the predictive validity of these instruments. In chapter 5 , we present a systematic review into the content, methodological quality, and effectiveness of intervention studies on the prevention of disability in community-dwelling physically frail elderly. In this review, the interest was particular in studies that included participants based on physical frailty indicators.

Chapter 6 reports a narrative review on the type of interventions that have been studied in randomized controlled and clinical controlled trials aimed at disability prevention in community-dwelling frail elderly. As we were interested in all kinds of interventions for frail elderly, this review was not restricted to physical frailty. Both the systematic review (chapter 5 ) and this review were used to summarize and identify promising components for future intervention programmes. Chapter 7 describes the content of the interdisciplinary disability prevention programme that we developed. It highlights programme's elements, its steps, tools, and 5 toolbox parts that comprise the intervention. Chapter 8 reports about the results of the feasibility study on our disability prevention programme. Finally, chapter 9 summarizes the main findings of our studies and discusses various methodological and theoretical aspects, followed by overall conclusions and implications for practice and future research. 


\section{REFERENCES}

1. European Commission. The 2009 Ageing Report: economic and budgetary projections for the EU-27 Member States (2008-2060). Luxembourg: Office for Official Publications of the European Communities; 2009.

2. SCP. Zorgen voor zorg: ramingen van de vraag naar personeel in de verpleging en verzorging tot 2030. Den Haag: Sociaal Cultureel Planbureau; 2010.

3. RIVM. Volksgezondheid Toekomst Verkenning, Nationaal Kompas Volksgezondheid. Bilthoven: Rijksinstituut voor Volksgezondheid en Milieu; 2009.

4. Gezondheidsraad. Preventie bij ouderen: focus op zelfredzaamheid. Den Haag: Gezondheidsraad; 2009.

5. Hogan DB, MacKnight C, Bergman H. Models, definitions, and criteria of frailty. Aging Clin Exp Res. 2003;15(3 Suppl):1-29.

6. Fried LP, Tangen CM, Walston J, Newman AB, Hirsch C, Gottdiener J, et al. Frailty in older adults: evidence for a phenotype. J Gerontol A Biol Sci Med Sci. 2001;56(3):M146-56.

7. Santos-Eggimann B, Cuenoud P, Spagnoli J, Junod J. Prevalence of frailty in middle-aged and older community-dwelling Europeans living in 10 countries. J Gerontol A Biol Sci Med Sci. 2009;64(6):67581.

8. Schuurmans H, Steverink N, Lindenberg S, Frieswijk N, Slaets JP. Old or frail: what tells us more? J Gerontol A Biol Sci Med Sci. 2004;59(9):M962-5.

9. Ministerie van VWS. Brief aan de Tweede Kamer. Multimorbiditeit en ouderenzorg. Den Haag: Ministerie van Volksgezondheid, Welzijn en Sport; 2007.

10. Abellan van Kan G, Rolland Y, Houles M, Gillette-Guyonnet S, Soto M, Vellas B. The Assessment of Frailty in Older Adults. Clin Geriatr Med 2010;26:275-86.

11. Bergman H, Ferrucci L, Guralnik J, Hogan DB, Hummel S, Karunananthan S, et al. Frailty: an emerging research and clinical paradigm--issues and controversies. J Gerontol A Biol Sci Med Sci. 2007;62(7):731-7.

12. Aminzadeh F, Dalziel W, Molnar F. Targeting frail older adults for outpatient comprehensive geriatric assessment and management services: An overview of concepts and criteria. Reviews in Clinical Gerontology. 2002;12:82-92

13. Lally F, Crome P. Understanding frailty. Postgrad Med J. 2007;83(975):16-20.

14. Saint Hubert de M, Swine C. Evolving definitions of frailty Aging Health. 2007;3(5):589-93.

15. De Lepeleire J, Degryse J, Illiffe S, Mann E, Buntinx F. Family physicians need easy instruments for frailty. Age Ageing. 2008;37(4):484; author reply -5 .

16. De Lepeleire J, Iliffe S, Mann E, Degryse JM. Frailty: an emerging concept for general practice. $\mathrm{Br} J$ Gen Pract. 2009;59(562):e177-82.

17. Hooi WC, Bergman H. A review on models and perspectives on frailty in older persons. SGH Proceedings. $2005 ; 14(2)$.

18. Jette AM. Toward a common language for function, disability, and health. Phys Ther. 2006;86(5):726-34.

19. Heikkinen $\mathrm{E}$. What are the main risk factors for disability in old age and how can disability be prevented? Copenhagen: WHO Regional Office for Europe; 2003.

20. Puts MT, Shekary N, Widdershoven G, Heldens J, Lips P, Deeg DJ. What does quality of life mean to older frail and non-frail community-dwelling adults in the Netherlands? Qual Life Res. 2007;16(2):263-77.

21. SCP. Rapportage ouderen 2006; Veranderingen in de leefsituatie en levensloop. Den Haag: Sociaal en Cultureel Planbureau; 2006.

22. Ministerie van VWS. Nota 'Ouderenbeleid in het perspectief van de vergrijzing'. Den Haag: Ministerie van Volksgezondheid, Welzijn en Sport; 2005.

23. Olde Rikkert MG. Ontwikkeling en onderzoek in de geriatrie: Quelques modes de traverser le mur de fragilité. Inaugurele rede. Nijmegen; 2004. 
24. KNMG. Sterke medische zorg voor kwetsbare ouderen. Utrecht: Koninklijke Nederlandse Maatschappij tot bevordering van de Geneeskunst; 2010.

25. LHV. Complexe ouderenzorg in verzorgingshuis en thuis. Utrecht: Landelijke Huisartsen Vereniging; 2009.

26. Schafer W, Kroneman M, Boerma W, van den Berg M, Westert G, Deville W, et al. The Netherlands: health system review. Health Syst Transit. 2010;12(1):v-xxvii, 1-228.

27. Knottnerus JA, ten Velden GH. Dutch doctors and their patients--effects of health care reform in the Netherlands. N Engl J Med. 2007;357(24):2424-6.

28. Oosterbos van $\mathrm{H}$. The future of integrated primary care; community health centres at the heart of the neighbourhood. International Journal of Integrated Care. 2006;6:1-2.

29. Schers H, Koopmans R, Olde Rikkert MG. De rol van de huisarts bij kwetsbare ouderen: een internetenquête onder huisartsen. Huisarts\&Wetenschap. 2009;52(13):626-30. 



\section{CHAPTER 2}

\section{Frailty in older age:}

Concepts and relevance for occupational and physical therapy

This chapter was published as:

Daniels R, van Rossum E, de Witte L, van den Heuvel W. Frailty in older age: Concepts and relevance for occupational and physical therapists. Physical and Occupational Therapy in Geriatrics 2008;27(2): 81-95. 


\section{Abstract}

Frailty refers to a state of vulnerability in older persons for adverse outcomes. The recent assumption that frailty, disability, and comorbidity are distinct concepts creates possibilities for interventions that focus on delaying the onset of disabilities. However, disagreement exists on the definition of frailty and the factors contributing to it. This article presents an overview of the discussion about the concept of frailty, of intervention research, and instruments to identify frail elderly, and discusses the implications for the fields of occupational and physical therapy. 


\section{Introduction}

In the next twenty years, the European Union will face a $30 \%$ increase in people over 65 and a 39\% increase in people over $80 .{ }^{1}$ As the prevalence of frailty is higher in older age ${ }^{2}$, the population of frail elderly will rise significantly. Frail elderly people are at a much higher risk of falls, infections, disabilities, hospitalization, institutionalization, and death, as compared with their age-matched non-frail counterparts. ${ }^{3,4}$ In scenarios that predict the future of health service delivery in the Western world, the rapid increase of frail elderly is seen as one of the major challenges for health care. $^{5,6}$

There has been an exponential rise in the use of the term 'frailty' in the literature. $^{7}$ This rise is accompanied by the development of a new paradigm in understanding frailty ${ }^{8}$ that points to opportunities for prevention and interventions aimed at either delaying the onset of frailty or reducing its adverse outcomes. ${ }^{9}$ This article presents an overview of the discussion about the concept of frailty, highlights instruments to identify frail elderly and discusses intervention research and the implications for the fields of occupational and physical therapy. A search for relevant articles (up to 2007) was conducted in various databases (Pubmed, Cinahl and CENTRAL) .

\section{Defining frailty}

Frailty is considered as a state of vulnerability towards adverse outcomes. Although everybody is vulnerable to a certain degree, it is old age that involves greater risks of exposure to challenges and, crucially, of reduced capacity to respond to these exposures. ${ }^{10}$ An important issue when considering vulnerability is whether there are objective criteria by which vulnerability can be assessed, or whether vulnerability is mainly a subjective experience. ${ }^{11}$ The term 'frail' or 'frailty' in reference to older adults was rarely used before the 1980s. ${ }^{7}$ In early definitions, frail denoted those aged 75 and older or individuals in need of special care. ${ }^{9}$ Chronic disease and its sequelae were felt to be the cause of functional limitations and frailty was another term for disability. ${ }^{7}$ From the 1990s, definitions of frailty which did not depend on the presence of chronic disease, dependency, or a need for health or social services began to appear. ${ }^{7}$ Nowadays, frailty, disability, and chronic disease(s) are considered to be distinct but overlapping concepts ${ }^{12}$ (see figure 1). Equating frailty with disability is inadequate as people with disabilities are not necessarily frail. ${ }^{13}$ 


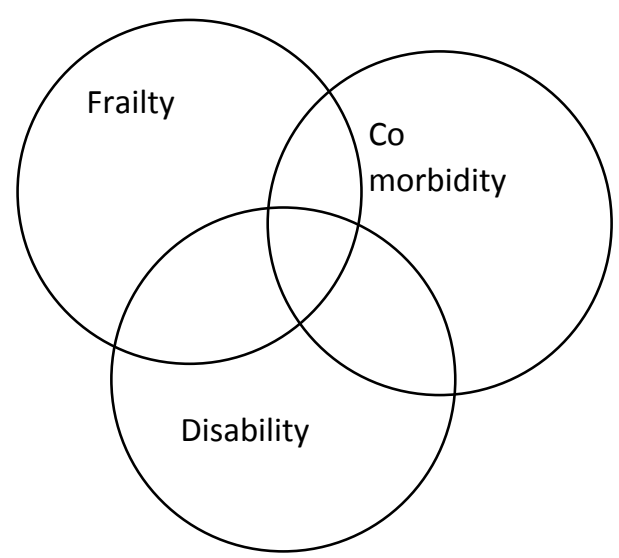

Figure 1 Relationship frailty, disability and comorbidity

Furthermore, not all frail elderly have disabilities: In a study of Fried et al. ${ }^{2}$, only $27 \%$ of the frail elderly experienced difficulties in ADL. The relationship between frailty and chronic disease(s) is complex. The development of acute and chronic diseases can precipitate frailty because they require the organism to mobilize the available resources with the potential consequence of exhausting the reserve function of organ systems. Frailty might just be the manifestation of clinical or undiagnosed chronic disease. ${ }^{14}$ However, Fried et al. ${ }^{2}$ found that only a small proportion of persons with at least two chronic diseases are frail, and some people with little or no disease show classic signs of frailty. It is suggested that it is actually frailty, as a core biologic dysregulation, that affects multiple organ systems and contributes to multiple aging-related diseases. ${ }^{8,15}$ The current literature on frailty supports the notion of a pathway to disability that is not a direct result of chronic disease, but is associated with age-related loss of physical condition and reserves. ${ }^{16}$ Whereas frailty used to be presented as a state of disability, it is nowadays considered as one of the pathways to disability ${ }^{7}$ and as a predictor of need for care and treatment. ${ }^{17}$ The conceptual distinction between frailty and disability creates possibilities to target older people in order to reverse or retard the progression of their condition. ${ }^{18}$

Although several definitions of frailty have been proposed, there is insufficient evidence yet to accept a single definition of frailty. ${ }^{19}$ The debate has focused on whether frailty should be defined purely in terms of biomedical factors or whether psychosocial factors should be included as well. ${ }^{20}$ From their literature reviews, Levers et al. ${ }^{21}$ as well as Aminzadeh et al. ${ }^{18}$ conclude that most definitions of frailty do include the idea of loss of age-related reserve capacity, though differences exist regarding other factors contributing to frailty. Moreover, there is substantial disagreement about the direction or nature of the relationship between frailty and the contributing factors. ${ }^{21}$ For clinical practice, frailty may emerge more sharply if it is described in biological terms. ${ }^{22} \mathrm{~A}$ consensus group of the American Geriatrics Soci- 
ety has settled on defining frailty as a physiologic syndrome characterized by decreased reserves and diminished resistance to stressors, resulting from cumulative decline across multiple physiologic systems. ${ }^{15}$ The often cited works of Fried supports the notion of frailty as a physiologic syndrome that can be identified and measured clinically. ${ }^{9}$ Fried et al. ${ }^{2}$ developed the 'frailty phenotype', in which individuals are considered frail if they meet at least three of the following five frailty markers: unintentional weight loss, muscle weakness, slow walking speed, exhaustion and low physical activity.

Still, this focus on 'physical frailty' is questioned for its usefulness in daily patient care in which vulnerabilities cannot be so easily separated from cognition, mood, and social support. ${ }^{23}$ Markle-Reid and Browne ${ }^{5}$ criticize the majority of the models for frailty for their view that frailty is uni-dimensional, predominantly biomedical in nature and characterised solely on the basis of functional losses that influence a person's capacity for independence in activities of daily living. The Canadian Initiative on Frailty and Aging ${ }^{16}$ considers frailty to be a measurable syndrome, though acknowledges that there is no universally accepted model or definition of frailty. This research group advocates for an integrative approach to frailty that includes biological, social, psychological and environmental components which interact across a person's lifespan and which may delay or promote the emergence of frailty (see Figure 2). The pathway from frailty to its adverse outcomes is affected by various modifiers which are described as the assets and deficits of an individual in a specific context. ${ }^{16}$

Do research outcomes support a specific definition of frailty? Several prospective studies showed that physical frailty markers have predictive value for the development of disability. ${ }^{24-26}$ Other studies, however ${ }^{27,28}$, support the view that frailty has to be studied as a complex interplay of physical, psychological, social, and environmental factors.

Rockwood $^{19}$ sees two different approaches when dealing with the variety of definitions of frailty. One approach is to accept a variety of viewpoints that can be classified, and as research proceeds, it should yield a definition clear enough to fit within an existing spectrum of definitions and be understood. Another is to propose one definition, see how it relates to earlier work, and, by means of consensus or compromise, to advocate strongly for it. This latter approach was chosen by the consensus group of the American Geriatrics Society ${ }^{15}$, which limited the definition of frailty to physical frailty. It may serve to simplify and expedite research, but whether it will help or hurt efforts to understand frailty is still an open question. ${ }^{29}$ 


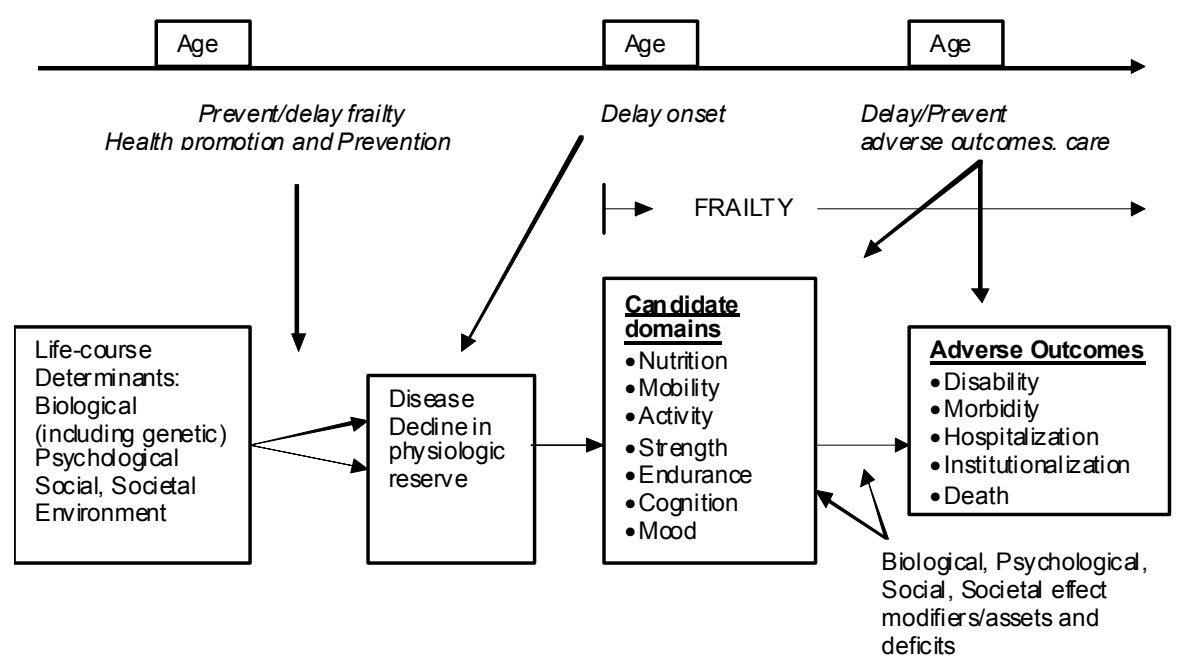

Figure 2 A possible working framework (Bergman et al., 2008)

\section{Identification of frail elderly}

The availability of tools to identify frail persons is relevant to both research and clinical purposes. To avoid costs and unnecessary assessment of healthy subjects, valid and low-cost tools are needed to screen elderly people who are particularly at risk of developing adverse outcomes. ${ }^{30}$ Several studies ${ }^{23,31-33}$ have focused on the validity, reliability and sensibility of frailty instruments. In general, a distinction can be made between instruments with criteria based on physical frailty ${ }^{2,34-38}$ and instruments based on a broad definition of frailty. ${ }^{6,23,31,32,39-45}$ Instruments are based on self-report, clinical observation, or a mix of the two. Furthermore, some instruments are designed for specific clinical settings, targeting, for example, elderly people at emergency departments. The previously mentioned five frailty markers as described by Fried et al. ${ }^{2}$ are based on a narrow definition of frailty. Performance measures such as balance, grip strength and gait velocity have been used as single criteria in clinical trials to select physically frail elderly. ${ }^{38}$ Instruments based on a broad definition are usually more extensive and include physical, cognitive, psychosocial and/or environmental factors. An example of such an instrument is the Edmonton Frail Scale which combines cognition, balance and mobility, mood, functional independence, medication use, social support, nutrition, healthy attitudes, continence, burden of medical illness, and quality of life. ${ }^{23}$ An overview of instruments is shown in Table 1. 
There is a need for further scientific work on the validity and reliability of frailty instruments. The five frailty markers showed in a prospective study ${ }^{2}$ the ability to select a frail population at high risk of adverse outcomes. Comparison of frailty instruments can show considerable convergence. Rockwood et al. ${ }^{46}$ found that an increase in each of Fried et al.'s five frailty markers correlated with an increased score on the Frailty Index, an instrument that considers frailty as deficit accumulation. However, another study indicated that various criteria for frailty give very different selections of elderly people. The prevalence of frailty in a sample of 125 elderly people admitted to a hospital differed from 36\% (grip strength), to $62 \%$ (criteria of Fried et al.), to $48 \%$ (Frailty Scale ${ }^{47}$ ) to $88 \%$ (gait velocity), depending on the criteria used. ${ }^{48}$ Before frailty criteria can be used to select frail elderly, each of the available criteria should be prospectively validated. ${ }^{48}$

\section{Interventions}

Intervention research in the field of frail elderly is relatively new. Aminzadeh et al. ${ }^{18}$ concluded that outpatient comprehensive geriatric assessment (CGA) and follow-up have many potential benefits for frail elderly, though their review revealed a lack of consensus for an explicit conceptual definition of frailty, standardized targeting criteria and validated practical screening criteria to select appropriate candidates effectively. The results of trials using preventive home visits by public health nurses, a form of CGA, are very mixed ${ }^{49,50}$.

Stuck and Kane ${ }^{51}$ suggest that, for older persons at low risk, some potential benefit may be expected from home-visiting programmes with a multidimensional approach. A number of interventions stem from the idea that many of the causes of frailty are treatable and even reversible through a combination of appropriate medical treatment and maintenance of a good diet and exercise regime. ${ }^{4,52,53}$

For instance, pharmacologic interventions are believed to be promising in frailty, although so far no evidence for the effect of hormonal and antiinflammatory interventions has been found. ${ }^{3}$ Lately, an increase in studies with a focus on physical exercise and nutrition can be noticed. ${ }^{54-61}$ Although improvements on physical measures such as strength, balance or gait speed are often reported, only a few studies ${ }^{59,62,63}$ demonstrated positive outcomes at the level of disability. The study of Gill et al. ${ }^{59}$, an in-home individualized programme focusing on physical and environmental conditions, showed benefits for moderately frail elderly, but not for severely frail elderly. The programme included instruction in safety techniques, proper use of devices, removal of environmental hazards and exercises for range of motion, balance, muscle conditioning and strengthening. 
Table 1 Frailty assessment instruments reported in literature

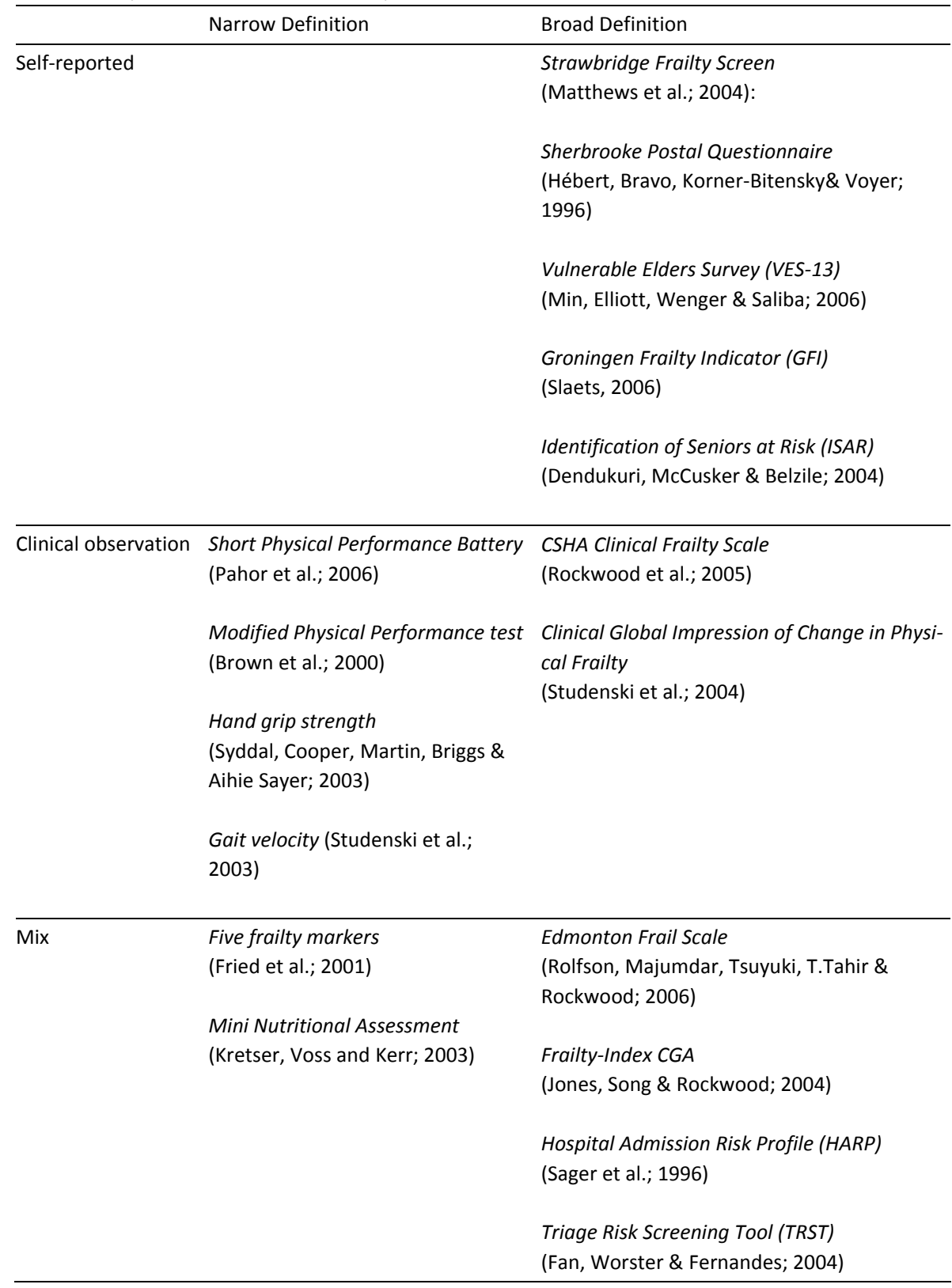

Furthermore, some studies with self-management programmes for frail elderly showed positive outcomes on well-being ${ }^{64}$, and inpatient hospital days. ${ }^{65}$ There are also indications that the use of assistive devices, home modifications, and smart technology can slow down functional decline in frail elderly. ${ }^{66,67}$ Some studies combined occupational and physical therapy. Gitlin et al. ${ }^{68,69}$ found that in-home occu- 
pational therapy and physical therapy in functionally vulnerable older people resulted in reduced mortality and increased functional status. Control-oriented strategies, including the use of environmental modifications and behavioural and cognitive strategies, were used in combination with balance, muscle strengthening, and safe fall and recovery training to optimize performance and compensate for declining abilities. Sanford et al. ${ }^{70}$ reported improved self-efficacy in mobility-impaired elderly by occupational and physical therapy sessions delivered in the traditional home setting or via tele-technology. Their study suggests that tele-technology can play a vital role in care for frail elderly.

\section{Implications for occupational and physical therapy}

Biomedical researchers have put frailty on the research agenda since the 1980s and their models shed a different light on disability prevention. There is, however, growing criticism towards the biomedical models in directing research to 'frailty equals physical frailty'. ${ }^{19}$ Restricting frailty to physical loss may lead to fragmentation of care with lack of attention to the whole person. ${ }^{5}$ The increased interest in frailty has alerted social researchers that this issue needs to become their focus as well. ${ }^{71}$ To reach an understanding of frailty and, if possible, a clear definition of frailty, research from several (including occupational) perspectives is necessary. As long as there is no consensus about frailty, researchers should make clear what they mean when they use the word frailty, and should continue to explore how each definition contributes to our overall understanding. ${ }^{72}$ Also, for practitioners, it is important to clarify their understanding of frailty. Since frailty is associated with disability, hospitalization and nursing home admission, occupational and physical therapists will increasingly see frail elderly people and can play a major role in their identification. Although there is no golden standard for the identification of frail elderly yet, there are a number of instruments that can be explored for their feasibility in daily care.

The use of different definitions of frailty in studies and the mixed results hamper the interpretation of effectiveness of interventions for frail elderly. Outcomes of clinical trials indicate that interventions for frail elderly need to address a complex of physical, behavioural, environmental and social factors. Single-component standard interventions are probably not effective. A multi-factorial approach, individualized to the needs of the frail older person, with multidisciplinary assessment that should reveal why this particular older person has an increased risk might be the way forward. Such a multi-factorial approach to frailty would better match the perception of successful ageing of older adults, involving beliefs about physical, functional, psychological and social health. ${ }^{73}$ Both occupational and physical therapy can make considerable contributions to the prevention of adverse outcomes in frail elderly. 
The conceptual distinction between frailty and disability shows a potential for interventions targeting frail elderly before experiencing (severe) disabilities, indicating that early involvement of occupational and physical therapy might be a crucial factor for the effectiveness of interventions.

\section{Conclusions}

Research on frailty is in an early stage of development. The current assumption is that (early) manifestations of frailty can be prevented or delayed and that the development of effective prevention and treatment is warranted. The debate about the concept of frailty, about valid instruments, and effective interventions is ongoing. There are indications that prevention and treatment should be multifactorial and multidisciplinary in nature. Occupational and physical therapists can play a major role in the identification of frail elderly and in the prevention of adverse outcomes. 


\section{References}

1. Heikkinen E. What are the main risk factors for disability in old age and how can disability be prevented? Copenhagen: WHO Regional Office for Europe (Health Evidence Network report;; 2003. Contract.

2. Fried LP, Tangen CM, Walston J, Newman AB, Hirsch C, Gottdiener J, et al. Frailty in older adults: evidence for a phenotype. J Gerontol A Biol Sci Med Sci. 2001;56(3):M146-56.

3. Espinoza S, Walston JD. Frailty in older adults: insights and interventions. Cleve Clin J Med. 2005;72(12):1105-12.

4. Storey E, Thomas RL. Understanding and ameliorating frailty in the elderly. Topics in Geriatric Rehabilitation. 2004;20(1):4-13.

5. Markle-Reid M, Browne G. Conceptualizations of frailty in relation to older adults. J Adv Nurs. 2003;44(1):58-68.

6. Slaets JP. Vulnerability in the elderly: frailty. Med Clin North Am. 2006;90(4):593-601.

7. Hogan DB, MacKnight C, Bergman H. Models, definitions, and criteria of frailty. Aging Clin Exp Res. 2003;15(3 Suppl):1-29.

8. Ferrucci L, Fried LP, editors. Frailty in Older Persons. Third IUSSP seminar on longevity and health; 2003; New York.

9. Hooi WC, Bergman H. A review on models and perspectives on frailty in older persons. SGH Proceedings. $2005 ; 14(2)$.

10. Grundy E. Ageing and vulnerable elderly people: European perspectives. Ageing and Society. 2006;26(1):105-34.

11. Schröder-Butterhill EM, R. A framework for understanding old-age vulnerabilities. Ageing and Society. 2006;26(1):9-35.

12. Fried LP, Ferrucci L, Darer J, Williamson JD, Anderson G. Untangling the concepts of disability, frailty, and comorbidity: implications for improved targeting and care. J Gerontol A Biol Sci Med Sci. 2004;59(3):255-63.

13. Bergman $H$, Hogan $D$, Karunananthan S. La fragilité. In: Arcand M, Hébert R, editors. Précis pratique de gériatrie. St-Hyacinthe and Paris: Edisem Maloine; 2007. p. 83-91.

14. Bergman H, Ferrucci L, Guralnik J, Hogan DB, Hummel S, Karunananthan S, et al. Frailty: an emerging research and clinical paradigm--issues and controversies. J Gerontol A Biol Sci Med Sci. 2007;62(7):731-7.

15. Walston J, Hadley EC, Ferrucci L, Guralnik JM, Newman AB, Studenski SA, et al. Research agenda for frailty in older adults: toward a better understanding of physiology and etiology: summary from the American Geriatrics Society/National Institute on Aging Research Conference on Frailty in Older Adults. J Am Geriatr Soc. 2006;54(6):991-1001.

16. Bergman H, Béland F, Karunananthan S, Hummel S, Hogan D, Wolfson C. Développement d'un cadre de travail pour comprendre et étudier la fragilité. Gérontologie et Société. 2004;109:15-29.

17. Hallberg IR, Kristensson J. Preventive home care of frail older people: a review of recent case management studies. J Clin Nurs. 2004;13(6B):112-20.

18. Aminzadeh F, Byszewski A, Dalziel WB, Wilson M, Deane N, Papahariss-Wright S. Effectiveness of outpatient geriatric assessment programs: exploring caregiver needs, goals, and outcomes. J Gerontol Nurs. 2005;31(12):19-25.

19. Rockwood K. What would make a definition of frailty successful? Age Ageing. 2005;34(5):432-4.

20. Lally F, Crome P. Understanding frailty. Postgrad Med J. 2007;83(975):16-20.

21. Levers MJ, Estabrooks CA, Ross Kerr JC. Factors contributing to frailty: literature review. J Adv Nurs. 2006;56(3):282-91.

22. Hamerman D. Toward an understanding of frailty. Ann Intern Med. 1999;130(11):945-50.

23. Rolfson DB, Majumdar SR, Tsuyuki RT, Tahir A, Rockwood K. Validity and reliability of the Edmonton Frail Scale. Age Ageing. 2006;35(5):526-9. 
24. Hardy SE, Dubin JA, Holford TR, Gill TM. Transitions between states of disability and independence among older persons. Am J Epidemiol. 2005;161(6):575-84.

25. Gill TM, Allore H, Holford TR, Guo Z. The development of insidious disability in activities of daily living among community-living older persons. Am J Med. 2004;117(7):484-91.

26. Boyd CM, Xue QL, Simpson CF, Guralnik JM, Fried LP. Frailty, hospitalization, and progression of disability in a cohort of disabled older women. Am J Med. 2005;118(11):1225-31.

27. Woo J, Goggins W, Sham A, Ho SC. Social determinants of frailty. Gerontology. 2005;51(6):402-8.

28. Ostir GV, Ottenbacher KJ, Markides KS. Onset of frailty in older adults and the protective role of positive affect. Psychol Aging. 2004;19(3):402-8.

29. Fisher AL. Just what defines frailty? J Am Geriatr Soc. 2005;53(12):2229-30.

30. Puts MT, Lips P, Deeg DJ. Static and dynamic measures of frailty predicted decline in performancebased and self-reported physical functioning. J Clin Epidemiol. 2005;58(11):1188-98.

31. Studenski S, Hayes RP, Leibowitz RQ, Bode R, Lavery L, Walston J, et al. Clinical Global Impression of Change in Physical Frailty: development of a measure based on clinical judgment. J Am Geriatr Soc. 2004;52(9):1560-6.

32. Jones DM, Song $X$, Rockwood K. Operationalizing a frailty index from a standardized comprehensive geriatric assessment. J Am Geriatr Soc. 2004;52(11):1929-33.

33. Steverink N, Slaets JPJ, Schuurmans H, Lis van M. Measuring Frailty. Development and testing of the Groningen Frailty Indicator (GFI). Gerontologist. 2001;41:236-7.

34. Brown M, Sinacore DR, Ehsani AA, Binder EF, Holloszy JO, Kohrt WM. Low-intensity exercise as a modifier of physical frailty in older adults. Arch Phys Med Rehabil. 2000;81(7):960-5.

35. Kretser AJ, Voss T, Kerr WW, Cavadini C, Friedmann J. Effects of two models of nutritional intervention on homebound older adults at nutritional risk. Journal of the American Dietetic Association. 2003;103(3):329-36.

36. Pahor M, Blair SN, Espeland M, Fielding R, Gill TM, Guralnik JM, et al. Effects of a physical activity intervention on measures of physical performance: Results of the lifestyle interventions and independence for Elders Pilot (LIFE-P) study. J Gerontol A Biol Sci Med Sci. 2006;61(11):1157-65.

37. Studenski S, Perera S, Wallace D, Chandler JM, Duncan PW, Rooney E, et al. Physical performance measures in the clinical setting. J Am Geriatr Soc. 2003;51(3):314-22.

38. Syddall H, Cooper C, Martin F, Briggs R, Aihie Sayer A. Is grip strength a useful single marker of frailty? Age Ageing. 2003;32(6):650-6.

39. Dendukuri N, McCusker J, Belzile E. The identification of seniors at risk screening tool: further evidence of concurrent and predictive validity. J Am Geriatr Soc. 2004;52(2):290-6.

40. Fan J, Worster A, Fernandes CM. Predictive validity of the triage risk screening tool for elderly patients in a Canadian emergency department. Am J Emerg Med. 2006;24(5):540-4.

41. Hebert R, Bravo G, Korner-Bitensky N, Voyer L. Predictive validity of a postal questionnaire for screening community-dwelling elderly individuals at risk of functional decline. Age Ageing. 1996;25(2):159-67.

42. Matthews M, Lucas A, Boland R, Hirth V, Odenheimer G, Wieland D, et al. Use of a questionnaire to screen for frailty in the elderly: an exploratory study. Aging Clin Exp Res. 2004;16(1):34-40.

43. Min LC, Elliott MN, Wenger NS, Saliba D. Higher vulnerable elders survey scores predict death and functional decline in vulnerable older people. J Am Geriatr Soc. 2006;54(3):507-11.

44. Rockwood K, Song X, MacKnight C, Bergman H, Hogan DB, McDowell I, et al. A global clinical measure of fitness and frailty in elderly people. Cmaj. 2005;173(5):489-95.

45. Sager MA, Rudberg MA, Jalaluddin M, Franke T, Inouye SK, Landefeld CS, et al. Hospital admission risk profile (HARP): identifying older patients at risk for functional decline following acute medical illness and hospitalization. J Am Geriatr Soc. 1996;44(3):251-7.

46. Rockwood K, Andrew A,Mitnitski A,. A comparison of two approaches to measuring frailty in elderly people. Journal of Gerontological Series A: Biological Sciences and Medical Sciences. 2007;62(7):738-43. 
47. Rockwood K, Stadnyk K, MacKnight C, McDowell I, Hebert R, Hogan DB. A brief clinical instrument to classify frailty in elderly people. Lancet. 1999;353(9148):205-6.

48. van lersel MB, Rikkert MG. Frailty criteria give heterogeneous results when applied in clinical practice. J Am Geriatr Soc. 2006;54(4):728-9.

49. van Haastregt JC, Diederiks JP, van Rossum E, de Witte LP, Crebolder HF. Effects of preventive home visits to elderly people living in the community: systematic review. Bmj. 2000;320(7237):754-8.

50. Stuck AE, Egger M, Hammer A, Minder CE, Beck JC. Home visits to prevent nursing home admission and functional decline in elderly people: systematic review and meta-regression analysis. Jama. 2002;287(8):1022-8.

51. Stuck A, Kane RL. Whom do preventive home visits help? J Am Geriatr Soc. 2008;56(3):561-3.

52. Bales CW, Ritchie CS. Sarcophenia, weight loss, and nutritional frailty in the elderly. AnnuRevNutr. 2002;22:309-23.

53. Wilson JF. Frailty--and its dangerous effects--might be preventable. Ann Intern Med. 2004;141(6):489-92.

54. Villareal DT, Banks M, Sinacore DR, Siener C, Klein S. Effect of weight loss and exercise on frailty in obese older adults. Arch Intern Med. 2006;166(8):860-6.

55. Faber MJ, Bosscher RJ, Chin A Paw MJ, van Wieringen PC. Effects of exercise programs on falls and mobility in frail and pre-frail older adults: A multicenter randomized controlled trial. Arch Phys Med Rehabil. 2006;87(7):885-96.

56. Binder EF, Schechtman KB, Ehsani AA, Steger-May K, Brown M, Sinacore DR, et al. Effects of exercise training on frailty in community-dwelling older adults: results of a randomized, controlled trial. J Am Geriatr Soc. 2002;50(12):1921-8.

57. Binder EF, Yarasheski KE, Steger-May K, Sinacore DR, Brown M, Schechtman KB, et al. Effects of progressive resistance training on body composition in frail older adults: results of a randomized, controlled trial. J Gerontol A Biol Sci Med Sci. 2005;60(11):1425-31.

58. Chin A Paw MJ, de Jong N, Schouten EG, Hiddink GJ, Kok FJ. Physical exercise and/or enriched foods for functional improvement in frail, independently living elderly: a randomized controlled trial. Arch Phys Med Rehabil. 2001;82(6):811-7.

59. Gill TM, Baker DI, Gottschalk M, Peduzzi PN, Allore H, Van Ness PH. A prehabilitation program for the prevention of functional decline: effect on higher-level physical function. Arch Phys Med Rehabil. 2004;85(7):1043-9.

60. Boshuizen HC, Stemmerik L, Westhoff MH, Hopman-Rock M. The effects of physical therapists' guidance on improvement in a strength-training program for the frail elderly. J Aging Phys Act. 2005;13(1):5-22.

61. Timonen L, Rantanen T, Makinen E, Timonen TE, Tormakangas T, Sulkava R. Effects of a group-based exercise program on functional abilities in frail older women after hospital discharge. Aging Clin Exp Res. 2006;18(1):50-6.

62. Gill TM, Baker DI, Gottschalk M, Peduzzi PN, Allore H, Byers A. A program to prevent functional decline in physically frail, elderly persons who live at home. N Engl J Med. 2002;347(14):1068-74.

63. Worm CH, Vad E, Puggaard L, Stovring H, Lauritsen J, Kragstrup J. Effects of a multicomponent exercise program on functional ability in community-dwelling frail older adults. Journal of the aging and physical activity. 2001;(9):414-24.

64. Frieswijk N, Steverink N, Buunk BP, Slaets JP. The effectiveness of a bibliotherapy in increasing the self-management ability of slightly to moderately frail older people. Patient Educ Couns. 2006;61(2):219-27.

65. Leveille SG, Wagner EH, Davis C, Grothaus L, Wallace J, LoGerfo M, et al. Preventing disability and managing chronic illness in frail older adults: a randomized trial of a community-based partnership with primary care. J Am Geriatr Soc. 1998;46(10):1191-8.

66. Mann WC, Ottenbacher KJ, Fraas L, Tomita M, Granger CV. Effectiveness of assistive technology and environmental interventions in maintaining independence and reducing home care costs for the frail elderly. A randomized controlled trial. Arch Fam Med. 1999;8(3):210-7. 
67. Tomita MR, Mann WC, Stanton K, Tomita AD, Sundar V. Use of currently available smart home technology for frail elders; process and outcomes. Topics in geriatric rehabilitation. 2007;23(1):2434.

68. Gitlin LN, Hauck WW, Winter L, Dennis MP, Schulz R. Effect of an in-home occupational and physical therapy intervention on reducing mortality in functionally vulnerable older people: preliminary findings. J Am Geriatr Soc. 2006;54(6):950-5.

69. Gitlin LN, Winter L, Dennis MP, Corcoran M, Schinfeld S, Hauck WW. A randomized trial of a multicomponent home intervention to reduce functional difficulties in older adults. J Am Geriatr Soc. 2006;54(5):809-16.

70. Sanford JA, Griffiths PC, Richardson P, Hargraves K, Butterfield T, Hoenig H. The effects of in-home rehabilitation on task self-efficacy in mobility-impaired adults: A randomized clinical trial. J Am Geriatr Soc. 2006;54(11):1641-8.

71. Barrett P. A case for examining the social context of frailty in later life. Australasian Journal on Ageing. 2006;25(3):114-8.

72. Rockwood K, Andrew M, Mitnitski A. A comparison of two approaches to measuring frailty in elderly people. J Gerontol A Biol Sci Med Sci. 2007;62(7):738-43.

73. Phelan EA, Anderson LA, LaCroix AZ, Larson EB. Older adults' views of "successful aging"--how do they compare with researchers' definitions? J Am Geriatr Soc. 2004;52(2):211-6. 


\section{CHAPTER 3}

\section{The psychometric properties of three self-report screening instruments for identifying frail older people in the}

community

This chapter was published as:

Metzelthin S, Daniëls R, van Rossum E, de Witte L, van den Heuvel W, Kempen GIJM. The psychometric properties of three self-report screening instruments for identifying frail older people in the community. BMC Public Health 2010;10:176 


\begin{abstract}
Background: Frailty is highly prevalent in older people. Its serious adverse consequences, such as disability, are considered to be a public health problem. Therefore, disability prevention in community-dwelling frail older people is considered to be a priority for research and clinical practice in geriatric care. With regard to disability prevention, valid screening instruments are needed to identify frail older people in time. The aim of this study was to evaluate and compare the psychometric properties of three screening instruments: the Groningen Frailty Indicator (GFI), the Tilburg Frailty Indicator (TFI) and the Sherbrooke Postal Questionnaire (SPQ). For validation purposes the Groningen Activity Restriction Scale (GARS) was added.
\end{abstract}

Methods: A questionnaire was sent to 687 community-dwelling older people $(\geq 70$ years). Agreement between instruments, internal consistency, and construct validity of instruments were evaluated and compared.

Results: The response rate was $77 \%$. Prevalence estimates of frailty ranged from $40 \%$ to $59 \%$. The highest agreement was found between the GFI and the TFI (Cohen's kappa = 0.74). Cronbach's alpha for the GFI, the TFI and the SPQ was 0.73, 0.79 and 0.26 , respectively. Scores on the three instruments correlated significantly with each other (GFI - TFI, $r=0.87$; GFI $-\mathrm{SPQ}, r=0.47$; TFI $-\mathrm{SPQ}, r=0.42$ ) and with the GARS (GFI - GARS, $r=0.57$; TFI - GARS, $r=0.61$; SPQ - GARS, $r=0.46$ ). The GFI and the TFI scores were, as expected, significantly related to age, sex, education and income.

Conclusions: The GFI and the TFI showed high internal consistency and construct validity in contrast to the SPQ. Based on these findings it is not yet possible to conclude whether the GFI or the TFI should be preferred; data on the predictive values of both instruments are needed. The SPQ seems less appropriate for postal screening of frailty among community-dwelling older people. 


\section{Background}

Frailty is highly prevalent in older people. Up to $40 \%$ of older people can be considered as frail and an increasing trend can be expected. ${ }^{1}$ Next to its high prevalence, frailty is characterized by its seriousness as it is related to increased risk of adverse health outcomes such as disability. ${ }^{2-4}$ Disability is defined as difficulty or dependency in the execution of activities of daily living and it is associated with increased health service utilization and related costs. Frailty and disability are separate but overlapping concepts. On the one hand, frailty predicts disability. On the other hand, disability may well exacerbate frailty. ${ }^{5}$ With regard to a growing frail population and limited health care expenditures, disability in community-dwelling frail older people is suggested to be a public health problem. ${ }^{3}$ Therefore disability prevention in community-dwelling frail older people is considered to be a priority for research and clinical practice in geriatric care. ${ }^{6}$

Several authors emphasize a two-step approach in preventive interventions for community-dwelling frail older people, in which screening is followed by extensive assessment. With valid (screening) instruments to identify frail older people, this approach may avoid costs and the unnecessary assessment of healthy people. ${ }^{7,8}$ During the last few decades, various instruments, based on various definitions, have been developed to detect frailty. This has led to a diversity of prevalence estimates of frailty. ${ }^{4}$ Little is yet known about the reliability and validity of these instruments and no gold standard exists. Therefore, more insight into the psychometric properties of frailty instruments is relevant for geriatric care and research in this area. ${ }^{4}$

Frailty instruments have been developed from the point of view of different perspectives on frailty. ${ }^{9}$ From a physiological perspective physical frailty markers, such as unintentional weight loss or weakness (grip strength), are used to identify frail older people. ${ }^{5}$ Next to physical factors, a multifactorial perspective on frailty also takes psychological, social and environmental factors into account. ${ }^{10}$ An example of such an instrument is the Frailty Index ${ }^{11,12}$, which combines, for example, physical frailty markers such as weight loss and grip strength with other factors such as cognition, mood or limitations in (instrumental) activities of daily living. Frailty may be elaborated more sharply if it is described from a physiological perspective, however, the usefulness of this perspective in daily practice is questioned, as frailty cannot be separated from other factors such as cognition, mood or social support. ${ }^{9}$

Frailty instruments can be divided into self-report and performance-based instruments. ${ }^{9}$ It is assumed that performance-based instruments provide more precise and valid answers ${ }^{13,14}$. Although they are less influenced by socio-demographic variables, personality and cognitive and affective factors, they are more sensitive to 
non-response, changes in time and differences in the execution of activities. Furthermore, they are less easy to conduct and time-consuming ${ }^{13-15}$. Self-report measures are believed to be an efficient method for reaching large groups and for providing high response rates and reliable and valid answers. ${ }^{15}$

In this study, we present the psychometric properties of frailty instruments that define frailty from a multifactorial perspective and are applicable for postal screening of community-dwelling older people. Given this objective and the target population, the Groningen Frailty Indicator $(\mathrm{GFI})^{16}$, the Tilburg Frailty Indicator (TFI) ${ }^{17}$ and the Sherbrooke Postal Questionnaire (SPQ) ${ }^{8}$ were chosen. The GFI and the SPQ have been used in previous studies for the purpose of postal screening ${ }^{18-22}$, however, empirical evidence about the psychometric properties of the GFI, the TFI, and the SPQ is still scarce. The purpose of the present study was to evaluate and compare their psychometric properties.

\section{Methods}

\section{Study design and participants}

A cross-sectional study was conducted in a sample of 687 community-dwelling older people living in the areas of Limburg and Utrecht in the Netherlands. Older people were identified between November 2008 and April 2009 from the panels of three general practitioners (GPs). All persons aged 70 years or above from each of the panels were invited by these GPs to participate in the study and to fill in a short questionnaire. The questionnaire included the three frailty instruments (GFI, TFI, $\mathrm{SPQ}$ ) and an instrument that measures disability with respect to (instrumental) activities of daily living: the Groningen Activity Restriction Scale (GARS) ${ }^{23}$. After two weeks a reminder was sent to non-respondents. Participants of the study were well informed about the study in a patient information letter that accompanied the questionnaire. The information letter was formulated according to the guidelines of good clinical practice. Participants had to give written informed consent. The study did not require ethical approval. The postal procedure, including the questionnaire, has proven to be feasible for postal screening in a pilot study. ${ }^{24}$

\section{Data collection}

The three frailty instruments and the disability measure are briefly described below. For an overview of all items please see Appendix: Frailty Instruments: Overview of all items. 


\section{Measures}

The Groningen Frailty Indicator (GFI), developed by Steverink and colleagues ${ }^{16}$, is a screening instrument for determining the level of frailty. It consists of fifteen items and focuses on the loss of functions and resources in four domains of functioning: physical (nine items), cognitive (one item), social (three items) and psychological (two items). Most items can be answered with 'yes' or 'no'. For the cognitive and psychosocial items the option 'sometimes' is added. Scores on the GFI range from zero to fifteen. A total score of four or higher is considered as moderately to severely frail. ${ }^{16,21}$ A study by Steverink and colleagues ${ }^{16}$ suggested that the GFI is an internally consistent scale with positive indications for construct and clinical validity.

The Tilburg Frailty Indicator (TFI) has recently been described by Gobbens and colleagues ${ }^{17}$ and consists of two subscales. The first subscale (ten items) comprises determinants of frailty, for example, socio-demographic data and data about lifeevents and chronic diseases. Socio-demographic data (age, sex, educational level and income) were used for validation purposes. The analyses of psychometric properties focus on the second subscale, which determines the level of frailty. This subscale consists of fifteen items that are about physical (eight items), social (three items) and psychological factors (four items), including one item which is about cognition. Most items can be answered with 'yes' or 'no'. For the psychological items the option 'sometimes' is added. Scores for the TFI range from zero to fifteen. A score of five or higher is considered to be associated with frailty. ${ }^{17}$

The Sherbrooke Postal Questionnaire (SPQ) was developed by Hébert and colleagues ${ }^{8}$ and consists of six items aiming to identify frail older people in the community. The items focus on the physical (four items), social (one item) and cognitive (one item) domains of functioning. Items can be answered with 'yes' or 'no'. Scores range from zero to six. Those older persons scoring two or higher, or who do not respond to the questionnaire, are considered to have an increased risk for functional decline and therefore are assumed to be frail. It should be noted that in the present study non-respondents were excluded from the analyses. In a Canadian sample of community-dwelling older people, predictive validity with regard to functional decline has been found ${ }^{8}$. There are also indications for its predictive validity with regard to requirements for further assessment ${ }^{20}$, use of emergency services ${ }^{22}$ and mortality. ${ }^{19}$

The Groningen Activity and Restriction Scale (GARS) ${ }^{23}$ is a valid and reliable instrument and consists of two subscales. The first subscale is about activities of (ADL) (eleven items). The second subscale relates to instrumental activities of daily living (IADL) (seven items). Items can be answered on a four point scale ranging from 'Yes, I can do it fully independently without any difficulty' to 'No, I cannot do it fully inde- 
pendently; I can only do it with someone's help'. Scores range from 18 to 72 (total scale), from 11 to 44 (ADL subscale) and from 7 to 28 (IADL subscale). Higher scores indicate greater disability in activities of daily living.

\section{Statistical analysis}

First, to provide an overview of respondents' background characteristics, descriptive statistics were used. Secondly, the reliability was determined from agreement between instruments (Kappa statistic based on proposed cut-off points by original authors) and internal consistency. Cronbach's alpha coefficient was calculated to evaluate internal consistency of items. Cronbach's alpha produces the same result as the Kuder-Richardson Formula 20 (K-R-20), which can be used to assess the internal consistency for dichotomous items. ${ }^{25}$ Furthermore, corrected total-itemcorrelations were calculated.

Thirdly, to assess the validity, non-parametric tests were used as our data were not normally distributed. If less than $25 \%$ (GFI, TFI, SPQ) or $50 \%$ (GARS) ${ }^{23}$ of the items were missing, these were imputed by means of case mean substitution. ${ }^{26}$ If more items were missing, persons were excluded from the analysis for the particular scale. The construct validity was assessed using Spearman's rank correlation between the three frailty instruments, as the instruments were assumed to measure the same concept of frailty. Frailty and disability are strongly related concepts ${ }^{5}$, as frail older people have an increased risk of disability and disability exacerbates frailty $^{2,3,5}$. Substantial associations between frailty and disability were expected. Therefore, construct validity was also assessed by examining associations between frailty and disability, measured by means of the GARS (Spearman's rank correlation). However, correlations should not be too high, otherwise frailty instruments and the GARS would measure the same concept. Furthermore, frail older people were more likely to be older, female, less educated, and had lower incomes compared to their non-frail counterparts. ${ }^{27}$ Since the distribution of frailty scores was non-normally distributed, Mann-Whitney $U$ and Kruskal-Wallis tests were performed to evaluate differences in the distribution of frailty scores among groups with different background characteristics. ${ }^{28}$ For the dichotomous variable gender (female versus male) the Mann-Whitney $U$ test was applied. For categorical variables with more than two groups (age, education and income), Kruskal-Wallis tests were used to compare the distribution of frailty scores among groups.

All statistical analyses were performed using SPSS for Windows, version 16.0. The level of statistical significance was set at $p=0.05$ (two-tailed). For post-hoc pairwise comparisons a Bonferroni correction was applied, so all effects are reported at a $p=$ 0.02 level of significance (two-tailed) in the case of three groups (age, education, income). 


\section{Results}

\section{Participants}

Of the 687 community-dwelling older people ( $\geq 70$ years), 532 (77.4\%) returned the questionnaire. The sample consisted of 311 women (58.5\%) and 221 men (41.5\%). In total, $64 \%$ of respondents lived in an urban area (Roermond, Amersfoort), while $36 \%$ lived in a rural area (Roggel). When using the proposed cut-off points, the GFI detected 245 frail cases (46.3\%). The TFI and the SPQ identified 211 (40.2\%) and 305 (59.1\%) frail older people, respectively. The mean age of respondents was 77.2 years with a range of $70-97$ years (SD $=5.5)$. Nearly half of the sample $(48.6 \%)$ had a secondary educational level. The largest proportion of people $(42.4 \%)$ had a net income of more than $€ 1500$ (per month/ per household). An overview of background characteristics is presented in Table 1. The sample is representative for the Dutch population of older people. According to a report of the Netherlands Institute for Social Research ${ }^{29}$ slightly more people aged 75 years and older are female. Older people, especially women, are often less educated and have an average income of about $€ 1500$ (per month/ per household).

Table 1 Characteristics of the participants $(n=532)$

\begin{tabular}{|c|c|c|c|c|}
\hline & & $\begin{array}{l}\text { Men } \\
(n=221)\end{array}$ & $\begin{array}{l}\text { Women } \\
(n=311)\end{array}$ & $\begin{array}{l}\text { Total } \\
(n=532)\end{array}$ \\
\hline Frail $^{\prime}$ & $\mathrm{n}(\%)$ & & & \\
\hline GFI & & $86(39.3)$ & $159(51.3)$ & 245 (46.3) \\
\hline TFI & & $66(30.1)$ & $145(47.4)$ & $211(40.2)$ \\
\hline SPQ & & $148(67.9)$ & $157(52.7)$ & 305 (59.1) \\
\hline Age $^{2}$ & $\mathrm{n}(\%)$ & & & \\
\hline $70-74$ yrs & & $91(41.2)$ & $102(32.8)$ & $193(36.3)$ \\
\hline 75-79 yrs & & $78(35.3)$ & $115(37.0)$ & $193(36.3)$ \\
\hline$\geq 80 \mathrm{yrs}$ & & $52(23.5)$ & $94(30.2)$ & $146(27.4)$ \\
\hline Education & n (\%) & & & \\
\hline $\begin{array}{l}\text { No Education/ Primary } \\
\text { Education }\end{array}$ & & $62(28.6)$ & $124(40.8)$ & $186(35.7)$ \\
\hline Secondary Education & & $102(47.0)$ & $151(49.7)$ & $253(48.6)$ \\
\hline Higher Education & & $53(24.4)$ & $29(9.5)$ & $82(15.7)$ \\
\hline Income & $\mathrm{n}(\%)$ & & & \\
\hline$\leq € 900$ & & $34(16.1)$ & $59(20.6)$ & $93(18.7)$ \\
\hline$€ 901$ to $€ 1500$ & & 63 (29.9) & $131(45.6)$ & $194(39.0)$ \\
\hline$\geq € 1501$ & & $114(54.0)$ & $97(33.8)$ & $211(42.4)$ \\
\hline
\end{tabular}

'Based on proposed cut-off points by original authors: GFI $\geq 4, T F I \geq 5, S P Q \geq 2$.

${ }^{2}$ Mean age men 76.6 years (SD 5.4), mean age women 77.6 years (SD 5.5). 
The frailty instrument with the greatest number of excluded respondents due to missing values (> 25\% missing values) was the SPQ ( $n=8)$. For the GFI and the TFI, one and two persons, respectively, were excluded due to missing values. On an item-level the number of missing values ranged from zero to eight (GFI), from zero to twelve (TFI) and from one to ten (SPQ). The average number of missing values per item was 2.4, 5.1 and 5.3 for the GFI, the TFI and the SPQ, respectively.

Table 2 Spearman correlation coefficients (99\%-confidence interval) among frailty instruments and GARS

\begin{tabular}{llllll}
\hline & GFI & TFI & SPQ & $\begin{array}{l}\text { GARS } \\
\text { Total scale }\end{array}$ & $\begin{array}{l}\text { GARS } \\
\text { ADL scale }\end{array}$ \\
\hline TFI & $\begin{array}{l}0.87 \\
(0.84-0.89)\end{array}$ & & & & \\
SPQ & 0.47 & 0.42 & & & \\
& $(0.1-0.55)$ & $(0.32-0.51)$ & & & \\
GARS & 0.57 & 0.61 & 0.46 & & \\
Total scale & $(0.49-0.64)$ & $(0.53-0.68)$ & $(0.37-0.54)$ & & \\
GARS & 0.54 & 0.58 & 0.41 & $(0.93-0.95)$ & \\
ADL scale & $(0.46-0.61)$ & $(0.5-0.65)$ & $(0.32-0.5)$ & 0.79 \\
GARS & 0.55 & 0.57 & 0.46 & $(0.96$ & $(0.74-0.83)$ \\
HDL scale & $(0.47-0.62)$ & $(0.49-0.64)$ & $(0.37-0.54)$ & $(0.95-0.97)$ & \\
\hline
\end{tabular}

\section{Reliability}

Cohen's Kappa coefficients between instruments were 0.74 (GFI - TFI), 0.28 (SPQ $\mathrm{GFI}$ ) and 0.25 (SPQ - TFI). According to Landis \& $\mathrm{Koch}^{30}$ the kappa values indicated good agreement between GFI and TFI and fair agreement between GFI and SPQ and between TFI and SPQ $(<0.20=$ poor, $0.21-0.40=$ fair, $0.41-0.60=$ moderate, 0.61 $-0.80=$ good, $0.81-1.00=$ very good agreement). Cronbach's alpha coefficients for the GFI, the TFI and the SPQ were $\alpha=0.73, \alpha=0.79$ and $\alpha=0.26$, respectively. The higher Cronbach's alpha, the more reliable the test is. Alpha values above 0.70 indicated a satisfactory internal consistency for a scale ${ }^{31}$. Corrected item-total correlations ranged from 0.14 to 0.55 with a mean of 0.30 (GFI), from 0.18 to 0.58 with a mean of 0.39 (TFI) and from 0.13 to 0.25 with a mean of 0.18 (SPQ).

\section{Validity}

Frailty instruments correlated significantly $(p<0.05)$ with each other and with disability measured by means of the GARS (convergent validity). The association between the GFI and the TFI scores was $r=0.87$. Correlations with the SPQ scores were $r=0.47$ for the GFI and $r=0.42$ for the TFI. The correlation coefficients between frailty instruments and disability (GARS) were $r=0.57$ (GFI - GARS), $r=0.61$ 
(TFI - GARS) and $r=0.46$ (SPQ - GARS). An overview of all correlation coefficients is presented in Table 2.

Table 3 shows the mean total scores and standard deviations of the GFI, the TFI and the SPQ related to (a) age, (b) sex, (c) education and (d) income. Scores on the GFI and the TFI were significantly higher for females, for persons with a higher age and for persons with lower education and lower incomes as compared to males, persons with a lower age, and persons with higher education and higher incomes. In contrast, on the SPQ we found higher scores among males as compared to females. Scores on the SPQ increased with higher age, lower education and lower incomes, however, the differences with respect to education and income were not significant ( $p=0.29$ and $p=0.08$ respectively).

Table 3 Mean scores on frailty instruments ' according to sex, age, educational level and income

\begin{tabular}{|c|c|c|c|c|}
\hline & & $\mathrm{GFI}$ & TFI & $\mathrm{SPQ}$ \\
\hline Total sample & Mean (sd) & $3.6(2.8)$ & $4.2(3.2)$ & $1.9(1.2)$ \\
\hline \multicolumn{5}{|l|}{$\overline{S e x}$} \\
\hline Male & & $3.2(2.7)$ & $3.4(3.1)$ & $2.1(1.2)$ \\
\hline \multirow[t]{3}{*}{ Female } & & $3.9(2.8)$ & $4.7(3.2)$ & $1.7(1.2)$ \\
\hline & Z statistic & -3.31 & -4.95 & -3.28 \\
\hline & (P-value) & $(0.001)$ & $(0.000)$ & $(0.001)$ \\
\hline \multicolumn{5}{|l|}{ Age } \\
\hline$\leq 74$ yrs & & $3.0(2.7)$ & $3.3(3.1)$ & $1.7(1.2)$ \\
\hline $75-79$ yrs & & $3.6(2.8)$ & $4.2(3.3)$ & $1.9(1.2)$ \\
\hline \multirow[t]{3}{*}{$\geq 80$ yrs } & & $4.4(2.7)$ & $5.3(3.1)$ & $2.2(1.2)$ \\
\hline & Chi-square $^{2}$ & 27.58 & 37.2 & 15.84 \\
\hline & (P-value) & $(0.000)$ & $(0.000)$ & $(0.000)$ \\
\hline \multicolumn{5}{|l|}{ Education } \\
\hline No Education/Primary & & $4.1(3.0)$ & $4.7(3.5)$ & $2.0(1.3)$ \\
\hline \multicolumn{5}{|l|}{ Education } \\
\hline Secondary Education & & $3.6(2.7)$ & $4.1(3.1)$ & $1.8(1.2)$ \\
\hline \multirow[t]{3}{*}{ Higher Education } & & $2.7(2.2)$ & $3.1(2.8)$ & $1.7(1.0)$ \\
\hline & Chi-square $^{2}$ & 12.13 & 13.47 & 2.47 \\
\hline & (P-value) & $(0.002)$ & $(0.001)$ & $(0.291)$ \\
\hline \multicolumn{5}{|l|}{ Income } \\
\hline$\leq € 900$ & & $4.6(3.0)$ & $5.3(3.6)$ & $2.2(1.4)$ \\
\hline$€ 901$ to $€ 1500$ & & $4.0(2.9)$ & $4.8(3.4)$ & $1.8(1.3)$ \\
\hline \multirow[t]{3}{*}{$\geq € 1501$} & & $2.8(2.3)$ & $3.1(2.6)$ & $1.8(1.0)$ \\
\hline & Chi-square $^{2}$ & 29.42 & 37.16 & 5.05 \\
\hline & (P-value) & $(0.000)$ & $(0.000)$ & $(0.080)$ \\
\hline
\end{tabular}

\footnotetext{
'Higher scores indicate poorer functioning.
}

${ }^{2}$ Kruskal Wallis test 


\section{Discussion}

The purpose of the present study was to evaluate and compare the psychometric properties of three screening instruments that define frailty from a multifactorial perspective and which are applicable for postal screening in community-dwelling frail older people. The chosen instruments were the Groningen Frailty Indicator (GFI), the Tilburg Frailty Indicator (TFI) and the Sherbrooke Postal Questionnaire (SPQ).

From the present study we may conclude that: (1) prevalence estimates of frailty ranged between $40.2 \%$ (TFI), 46.3\% (GFI) and 59.1\% (SPQ); (2) the agreement in identifying frailty between the GFI and the TFI was satisfactory (kappa $=0.74$ ) and the agreements between the SPQ and the GFI and the TFI, respectively, were much lower; (3) both the GFI and the TFI had high internal consistency in contrast to the SPQ; (4) the GFI and the TFI had better construct validity in comparison with the SPQ.

Prevalence estimates of $40 \%$ to $60 \%$ found in the present study can be considered as high. It is important to bear in mind that prevalence estimates strongly depend on the interpretation of the concept of frailty and the approach that is chosen to measure it. ${ }^{32}$ In a recent study by Santos-Eggimann and colleagues ${ }^{33}$, a distinction was made between frail and pre-frail older people based on the frailty phenotype of Fried and colleagues ${ }^{5,34}$. In a Dutch sample of community-dwelling older people, Santos-Eggimann and colleagues ${ }^{33}$ found a frailty prevalence of $11.3 \%$, while $38.5 \%$ were identified as pre-frail. These results indicate that the instruments in our study, based on the proposed cut-off points, may identify pre-frail instead of frail older people. Further research is needed to provide a better view on relevant cut-off points for frailty instruments. Longitudinal studies are needed to investigate the predictive power of instruments to identify older people who are at risk for adverse health outcomes in the near future.

Steverink and colleagues ${ }^{16}$ suggested that the GFI is an internally consistent scale with positive indications for construct and clinical validity. The present study supports these findings. Similar results for the TFI may be explained by seven out of fifteen items of the TFI being identical with the GFI. These items are about hearing and vision capacity, unintentional weight loss and psychosocial and cognitive functioning. Please see Appendix: Frailty Instruments: Overview of all items for more information about the instruments. Scores on the Sherbrooke Postal Questionnaire were higher for males compared with females. This finding is inconsistent with the literature. ${ }^{27}$ However, other findings on the Sherbrooke Questionnaire (higher score with higher age, lower educational level and lower incomes) are well in line with the literature. ${ }^{27}$ Previous studies about the SPQ have reported regarding the predictive validity of the SPQ ${ }^{8,19,20,22}$, however, in the present study the SPQ showed less reli- 
ability and construct validity. Conclusions about predictive validity can not be drawn for any of the three instruments.

The findings of the present study should be interpreted in the context of potential limitations. First, little is known about the test-retest reliability of the instruments. Second, there is no gold standard available as an external criterion of frailty. Future studies could analyse the predictive validity of the frailty instruments with respect to disability, health service utilization and mortality. Last, the SPQ was not fully used according to the protocol, as non-respondents were excluded from analyses. According to the protocol of the SPQ ${ }^{8}$, non-respondents should also be considered at risk (which would have resulted in a prevalence estimate of $67.0 \%$ instead of $59.1 \%)$. The strengths of the present study are the comparisons of the psychometric properties of the frailty instruments, the proven feasibility of the postal procedure ${ }^{24}$ and the response rate of $77.4 \%$, which is as good as, or even better than, previous studies in which postal screening procedures were applied. ${ }^{24,35,36}$

Although most older people may visit their GP regularly, primary care often fails in the identification of the health care needs of older people. ${ }^{37}$ Screening has the potential to identify older people at risk, followed by comprehensive assessment when needed. ${ }^{7,8}$ Frailty instruments have to provide reliable and valid answers and have to be feasible. ${ }^{15}$ The psychometric properties of the TFI were slightly better than those of the GFI. However, the number of missing values was lower for GFI items than for TFI items, indicating a higher feasibility of the GFI. Based on these findings it is not yet possible to conclude whether the GFI or the TFI should be preferred for postal screening. The SPQ is less appropriate with regard to its psychometric quality and missing values.

Future (longitudinal) research into the psychometric properties of the GFI and the TFI is urgently needed with regard to predictive validity and test-retest reliability of the GFI and the TFI. In addition, comparing the GFI and the TFI with other frailtyrelated constructs would lead to more insight into their construct validity.

\section{Conclusion}

Valid screening instruments for identifying community-dwelling frail older people are needed for disability prevention. The GFI and the TFI have shown high internal consistency and construct validity, in contrast to the SPQ. Prevalence estimates of frailty ranged from $40 \%$ to $59 \%$. Most agreement was found between the GFI and the TFI. Based on these findings, it is not possible to conclude whether the GFI or 
the TFI should be preferred for screening. The SPQ seems less appropriate. Further research is needed.

\section{Acknowledgements}

The authors are grateful to the patients for their willingness to participate in the present study. The Centre for Data and Information Management (MEMIC) at Maastricht University is acknowledged for their assistance in the data collection. This research has been funded by Zuyd University of Applied Sciences. 


\section{References}

1. Slaets JP. Vulnerability in the elderly: frailty. Med Clin North Am. 2006;90(4):593-601.

2. Levers MJ, Estabrooks CA, Ross Kerr JC. Factors contributing to frailty: literature review. J Adv Nurs. 2006;56(3):282-91.

3. Markle-Reid M, Browne G. Conceptualizations of frailty in relation to older adults. J Adv Nurs. 2003;44(1):58-68.

4. Pel Littel RE, Schuurmans MJ, Emmelot Vonk MH, Verhaar HJ. Frailty: defining and measuring of a concept. J Nutr Health Aging. 2009;13(4):390-4.

5. Fried LP, Ferrucci L, Darer J, Williamson JD, Anderson G. Untangling the concepts of disability, frailty, and comorbidity: implications for improved targeting and care. J Gerontol A Biol Sci Med Sci. 2004;59(3):255-63.

6. Daniels R, Metzelthin S, Van Rossum E, De Witte L, Van den Heuvel W. Interventions to prevent disability in frail community-dwelling older persons: an overview European Journal of Ageing. 2010;7(1):137-55.

7. De Lepeleire J, Degryse J, Illiffe S, Mann E, Buntinx F. Family physicians need easy instruments for frailty. Age Ageing. 2008;37(4):484; author reply -5 .

8. Hebert R, Bravo G, Korner-Bitensky N, Voyer L. Predictive validity of a postal questionnaire for screening community-dwelling elderly individuals at risk of functional decline. Age Ageing. 1996;25(2):159-67.

9. Daniels R, Van Rossum HIJ, De Witte LP, Van den Heuvel WJA. Frailty in Older Age: Concepts and Relevance for Occupational and Physical Therapy. Physical \& Occupational Therapy In Geriatrics. 2008;27:2:81 - 95.

10. Bergman H, Beland F, Karunanthan S, Hummel S, Hogan D, Wolfson C. Developing a working framework for understanding frailty. (English translation of article published in) Gerontologie et Societe. 2004;109:15-29.

11. Rockwood K, Mitnitski A. Frailty in relation to the accumulation of deficits. J Gerontol A Biol Sci Med Sci. 2007;62(7):722-7.

12. Searle SD, Mitnitski A, Gahbauer EA, Gill TM, Rockwood K. A standard procedure for creating a frailty index. BMC Geriatr. 2008;8:24.

13. Guralnik JM, Branch LG, Cummings SR, Curb JD. Physical performance measures in aging research. J Gerontol. 1989;44(5):M141-6.

14. Kempen GI, van Heuvelen MJ, van den Brink RH, Kooijman AC, Klein M, Houx PJ, et al. Factors affecting contrasting results between self-reported and performance-based levels of physical limitation. Age Ageing. 1996;25(6):458-64.

15. Martin FC, Brighton P. Frailty: different tools for different purposes? Age Ageing. 2008;37(2):129-31.

16. Steverink N, Slaets JPJ, Schuurmans H, Lis van M. Measuring Frailty. Development and testing of the Groningen Frailty Indicator (GFI). Gerontologist. 2001;41:236-7.

17. Gobbens RJJ, van Assen MALM, Luijkx KG, Wijnen-Sponselee MT, Schols JMGA. The Tilburg Frailty Indicator: Psychometric Properties. Journal of the American Medical Director Association. accepted for publication.

18. Frieswijk N, Steverink N, Buunk BP, Slaets JP. The effectiveness of a bibliotherapy in increasing the self-management ability of slightly to moderately frail older people. Patient Educ Couns. 2006;61(2):219-27.

19. Roberts H, Hemsley Z, Thomas G, Aihie Sayer A, Gove I, Turner G, et al. Can the Sherbrooke postal questionnaire predict who will require comprehensive assessment in the single assessment process? Society BG, editor. Birmingham, UK: British Geriatrics Society 2005.

20. Roberts HC, Hemsley ZM, Thomas G, Meakins P, Powell J, Robison J, et al. Nurse-led implementation of the single assessment process in primary care: a descriptive feasibility study. Age Ageing. 2006;35(4):394-8. 
21. Schuurmans H, Steverink N, Lindenberg S, Frieswijk N, Slaets JP. Old or frail: what tells us more? J Gerontol A Biol Sci Med Sci. 2004;59(9):M962-5.

22. Walker L, Jamrozik K, Wingfield D. The Sherbrooke Questionnaire predicts use of emergency services. Age Ageing. 2005;34(3):233-7.

23. Kempen GI, Miedema I, Ormel J, Molenaar W. The assessment of disability with the Groningen Activity Restriction Scale. Conceptual framework and psychometric properties. Soc Sci Med. 1996;43(11):1601-10.

24. Steinbusch C. Screening of frail elderly in the community. The feasibility and psychometric properties of three instruments. Maastricht: Maastricht; 2008.

25. Kuder GF, Richardson MW. The theory of the estimation of test reliability. Psychometrika. 1937;2:151-60.

26. Fox-Wasylyshyn SM, El-Masri, M.M. Focus on Research Methods Handling Missing Data in SelfReport Measures. Research in Nursing \& Health. 2005;28 488-95.

27. Avila-Funes JA, Helmer C, Amieva H, Barberger-Gateau P, Le Goff M, Ritchie K, et al. Frailty among community-dwelling elderly people in France: the three-city study. J Gerontol A Biol Sci Med Sci. 2008;63(10):1089-96.

28. Rosner B. Fundamentals of Biostatistics. 6th ed. Duxbury: Thomson Brooks/Cole; 2006.

29. Lokale en nationale monitor gezondheid. 2008 [cited]. Available from: http://www.monitorgezondheid.nl/home.xml.

30. Landis JR, Koch GG. The measurement of observer agreement for categorical data. Biometrics. 1977;33(1):159-74.

31. Nunnally JC. Psychometric theory 2nd edition ed. New York: McGraw-Hill; 1978.

32. Bergman H, Ferrucci L, Guralnik J, Hogan DB, Hummel S, Karunananthan S, et al. Frailty: an emerging research and clinical paradigm--issues and controversies. J Gerontol A Biol Sci Med Sci. 2007;62(7):731-7.

33. Santos-Eggimann B, Cuenoud P, Spagnoli J, Junod J. Prevalence of frailty in middle-aged and older community-dwelling Europeans living in 10 countries. J Gerontol A Biol Sci Med Sci. 2009;64(6):67581.

34. Fried LP, Tangen CM, Walston J, Newman AB, Hirsch C, Gottdiener J, et al. Frailty in older adults: evidence for a phenotype. J Gerontol A Biol Sci Med Sci. 2001;56(3):M146-56.

35. Bouman A. Home visiting program for older persons with poor health status [PhD thesis]. Maastricht: Maastricht University; 2008.

36. Zijlstra GAR. Managing concerns about falls. Fear of falling and avoidance of activity in older people. [PhD thesis]. Maastricht: Maastricht University; 2008.

37. Bowns I, Challis D, Tong MS. Case finding in elderly people: validation of a postal questionnaire. $\mathrm{Br} \mathrm{J}$ Gen Pract. 1991;41(344):100-4. 


\section{CHAPTER 4}

\section{The predictive validity of three self- report screening instruments for identifying frail older people in the}

community

This chapter was submitted as:

Daniels R, van Rossum E, van den Heuvel W, Beurskens S, de Witte L. The predictive validity of three self-report screening instruments for identifying frail older people in the community (2011) 


\begin{abstract}
Objective: To investigate the predictive values of three self-report instruments for identifying community-dwelling frail elderly.

Design: Prospective study with a 1-year follow-up

Setting: Community-based

Participants: Community-dwelling elderly aged 70 or older $(n=430)$

Measurements: The Groningen Frailty Indicator (GFI), Tilburg Frailty Indicator (TFI) and Sherbrooke Postal Questionnaire (SPQ) were tested for their predictive values on development of disabilities (measured by the Groningen Activity Restriction Scale), hospital admission and mortality.

Results: Adjusted odds ratios show that those identified as frail have more than twice the risk (GFI, 2.62; TFI, 2.00; SPQ, 2,49) for developing disabilities compared to the non-frail group; those identified as frail by the TFI and SPQ have more than twice the risk of being admitted to a hospital. Sensitivity and specificity for development of disabilities are $71 \%$ and $63 \%$ (GFI), $62 \%$ and $71 \%$ (TFI) and $83 \%$ and $48 \%$ (SPQ). Regarding mortality, sensitivity for all tools are about $70 \%$ and specificity between $41 \%$ and $61 \%$. For hospital admission, SPQ scores the highest for sensitivity (76\%).
\end{abstract}

Conclusions: All three instruments do have potential to identify older persons at risk, but their predictive power is not sufficient yet. Further research on these and other instruments is needed to improve targeting frail elderly. 


\section{Introduction}

The prevalence of frailty increases with age. Frail elderly have a higher risk of disabilities, fall incidents, hospitalization, institutionalization, and death compared to non-frail elderly. ${ }^{1}$ With regard to a growing frail population, prevention of adverse outcomes in community-dwelling frail older people is considered to be a priority for research and clinical practice in geriatric care. ${ }^{2}$

Effective screening of frailty is crucial in optimizing care to this vulnerable group. ${ }^{3}$ Although various tools have been developed, there is not yet a standardized and valid method to screen for frailty. Several authors ${ }^{4,5}$ emphasize a two-step approach in preventive interventions, in which screening is followed by extensive assessment. If brief and easy to use self report screening tools are available, this approach may avoid costs and unnecessary assessment of healthy people. It is, therefore, important to study whether tools can predict relevant outcomes associated with frailty. We focused on three available instruments for community-dwelling elderly care: the Groningen Frailty Indicator $(\mathrm{GFI})^{6}$, the Tilburg Frailty Indicator (TFI) ${ }^{7}$ and the Sherbrooke Postal Questionnaire (SPQ). ${ }^{5}$

All instruments were developed to screen for frailty as a first step in identification. In a Canadian sample of community-dwelling older people, sufficient predictive validity for the SPQ, with regard to functional decline, was reported. ${ }^{5}$ There are also positive indications for its predictive value with regard to requirements for further assessment ${ }^{8}$, use of emergency services ${ }^{9}$, and mortality. ${ }^{10}$ We found in a Dutch sample of community-dwelling older people that the GFI and TFI have high internal consistency and construct validity in contrast to the SPQ. ${ }^{11}$ In a cross-sectional study, Gobbens et al. ${ }^{7}$ reported strong associations between TFI scores and quality of life, disability, and use of nursing and informal care. No longitudinal studies on the predictive values of the GFI and TFI have been conducted yet. The aim of this study is to compare these values of the GFI, TFI and SPQ for relevant adverse outcomes in community-dwelling frail elderly: the development of disabilities, hospital admission, and mortality.

\section{Methods}

\section{Study design and participants}

A longitudinal study was conducted in a sample of 687 community-dwelling older people living in the areas of Limburg and Utrecht in the Netherlands. Older people were identified between November 2008 and April 2009 (T1) from the panels of 
four general practitioners (GPs). All persons aged 70 years or above from each of the panels were invited by their GP to fill in a questionnaire. The questionnaire included the three frailty instruments (GFI, TFI, SPQ) and an instrument that measures disability with respect to activities of daily living: the Groningen Activity Restriction Scale (GARS). ${ }^{12}$ After two weeks, a reminder was sent to non-responders. A pilot study showed that this postal procedure was feasible. ${ }^{13}$

One year later (T2), the same questionnaire was sent again to all participants who had provided written consent and responded to the first measurement, except those who had been admitted to a nursing home. This time a question about admission to a hospital in the previous year was added. The study did not require approval from an ethical committee according to Dutch law. Participants gave their written informed consent based on a patient information letter that accompanied the questionnaire. This letter was formulated according to guidelines of good clinical practice.

\section{Data collection}

The three frailty instruments and the disability measure are briefly described below. For an overview of all items of the three instruments see Appendix: Frailty Instruments: Overview of all items.

\section{Frailty instruments}

The Groningen Frailty Indicator (GFI), developed in the Netherlands by Steverink and colleagues ${ }^{6}$, is a screening instrument for determining the level of frailty. It consists of fifteen items, and focuses on the loss of functions and resources in four domains of functioning: physical (nine items), cognitive (one item), social (three items), and psychological (two items). Most items can be answered with 'yes' or 'no'. For the cognitive and psychosocial items, the option 'sometimes' is added. Scores on the GFI range from zero to fifteen. A total score of four or higher is considered as moderately to severely frail. ${ }^{6,14}$

The Dutch Tilburg Frailty Indicator (TFI) has recently been described by Gobbens and colleagues ${ }^{7}$ and consists of two subscales. The first subscale (ten items) comprises socio-demographic data and data about life-events and chronic diseases. The analyses of predictive values focuses on the second subscale, which determines the level of frailty. This subscale consists of fifteen items about physical (eight items), social (three items), and psychological factors (four items), including one item about cognition. Most items can be answered with 'yes' or 'no'. For the psychological items, the option 'sometimes' is added. Scores for the TFI range from zero to fifteen. A score of five or higher is considered to be associated with frailty. ${ }^{7}$ 
The Sherbrooke Postal Questionnaire (SPQ) was developed in Canada by Hébert and colleagues ${ }^{5}$ and consists of six items. These focus on the physical (four items), social (one item), and cognitive (one item) domains of functioning. Items can be answered with 'yes' or 'no'. Scores range from zero to six. Those scoring two or higher, or who do not respond to the questionnaire, are considered to have an increased risk for functional decline and, therefore, are assumed to be frail. It should be noted that in the present study non-responders were excluded from the analyses.

\section{Outcome measures}

The Groningen Activity and Restriction Scale (GARS) ${ }^{12}$ is a valid and reliable instrument for measuring disability. The first subscale is about activities of daily living (ADL) (eleven items). The second subscale relates to instrumental activities of daily living (IADL) (seven items). Items can be answered on a four point scale. In line with the GARS manual ${ }^{15}$, the items were dichotomized into being independent or being dependent regarding performance of an activity. Development of disability was defined as (at least) one new disability, meaning a change on at least one of the 18 items of the GARS from being independent to being dependent. Regarding hospital admission, we asked "have you been admitted to a hospital in the previous year". A hospital stay, for at least one day, was regarded as hospital admission. The GP provided data at $\mathrm{T} 2$ about persons who had died during follow up.

\section{Statistical analysis}

All statistical analyses were performed using SPSS for Windows, version 18.0. In preparing data, missings on the $\mathrm{GFI}, \mathrm{TFI}$, and SPQ (if less than $25 \%$ of all scale items) on $\mathrm{T} 1$ were imputed by means of case mean substitution. ${ }^{16}$ To investigate whether baseline characteristics of participants who were lost to follow-up differed from those who remained in the sample, the independent samples t-tests, chi-square tests and Kendall's tau-c tests were used. The areas under the receiver operating characteristic (ROC) curve (AUC) for the proposed cut off points were calculated to compare the accuracy of the instruments. With these points sensitivity, specificity, and positive and negative predicted values were determined for development of disabilities, mortality, and hospital admission. To avoid ceiling effects in calculating predictive values regarding development of disabilities, those scoring 60 or higher on the GARS at T1 $(n=4)$ were removed from analyses. Odds ratios (OR) were calculated to compare frail versus non-frail groups for their risk for the adverse outcomes. OR's adjusted for age, sex, GARS score on T1, education, and income were calculated using logistic regression (fixed model). 


\section{Results}

Of the 687 elderly people who were invited, 532 (77\%) returned the baseline postal questionnaire and gave written consent. One year later, the same questionnaire was sent to 514 of these 532 participants (15 participants had died and three had been admitted to a nursing home); 440 (86\%) returned the second questionnaire. Ten participants were excluded from the analysis, because different persons filled out the questionnaires at T1 and T2. Finally, 430 participants were included in the analyses (63\% of the original sample) for calculating the predictive values for developing disabilities and hospital admissions (for mortality analysis $n=532$ ).

Table 1 Patient characteristics

\begin{tabular}{|c|c|c|c|c|}
\hline & $\begin{array}{l}\text { T1 } \\
(n=532)^{*}\end{array}$ & $\begin{array}{l}\text { T2 } \\
(n=430)^{*}\end{array}$ & $\begin{array}{l}\text { Non-responders } \\
(n=74)^{*}\end{array}$ & $\begin{array}{l}\text { Significance-level } \\
\text { difference respond- } \\
\text { ers/non-responders } \\
{\text { at } \mathrm{T}^{+}}^{+}\end{array}$ \\
\hline \multicolumn{5}{|l|}{ Age } \\
\hline $70-74$ & $193(36.3)$ & $158(36.7)$ & $24(32.4)$ & $p=0.46$ \\
\hline $75-79$ & $193(36.3)$ & $157(36.5)$ & $28(37.8)$ & \\
\hline$\geq 80$ & $146(27.4)$ & $115(26.7)$ & $22(29.7)$ & \\
\hline Women & $311(58.5)$ & $259(60.2)$ & $39(52.7)$ & $p=0.22$ \\
\hline \multicolumn{5}{|l|}{ Education } \\
\hline None/primary education & $186(35.7)$ & 140 (32.9) & 31 (41.9) & $p=0.05$ \\
\hline Secondary education & $253(48.6)$ & $213(50.1)$ & $30(40.5)$ & \\
\hline Higher education & $82(15.7)$ & $72(16.9)$ & $8(10.8)$ & \\
\hline \multicolumn{5}{|l|}{ Income } \\
\hline$\leq 900$ & $93(18.7)$ & $64(15.6)$ & $24(32.4)$ & $\mathrm{p}<0.001$ \\
\hline $901-1500$ & $194(39.0)$ & $157(38.4)$ & $28(37.8)$ & \\
\hline$\geq 1501$ & $211(42.4)$ & $188(46.0)$ & $15(20.3)$ & \\
\hline \multicolumn{5}{|l|}{ Frail } \\
\hline $\mathrm{GFI}(\geq 4)$ & $245(46.3)$ & $198(46.0)$ & $32(43.2)$ & $p=0.63$ \\
\hline $\mathrm{TFI}(\geq 5)$ & $211(40.2)$ & $162(38.2)$ & 35 (47.9) & $p=0.12$ \\
\hline$S P Q(\geq 2)$ & $305(59.1)$ & $250(58.1)$ & $37(52.9)$ & $p=0.28$ \\
\hline \multicolumn{5}{|l|}{ Disability (mean) } \\
\hline GARS total (18-72) & 24.9 (sd 9.3) & $24.2(\mathrm{sd} 8.3)$ & $28.2(\mathrm{sd} 12.6)$ & $\mathrm{p}<0.001$ \\
\hline GARS-ADL (11-44) & $13.9(\mathrm{sd} 4.5)$ & 13.5 (sd 3.9) & 15.6 (sd 6.5) & $p<0.001$ \\
\hline GARS-HDL (7-28) & $11.1(\mathrm{sd} 5.4)$ & 10.7 (sd 4.9) & 12.7 (sd 6.7) & $\mathrm{p}<0.001$ \\
\hline Mortality & - & $15(2.8 \%)$ & - & \\
\hline Hospital admission & - & 75 (17.4\%) & - & \\
\hline
\end{tabular}

* Due to missings small differences between $\mathrm{n}$ and numbers of participant reported for education and income can occur

† Comparison of these subgroups on baseline scores 
Characteristics of participants at $\mathrm{T} 1$ and $\mathrm{T} 2$, as well as non-responders at $\mathrm{T} 2$, are listed in table 1 . The mean age at $\mathrm{T} 1$ was 77.2 years $(\mathrm{SD}=5.5)$ and about $60 \%$ were women.

Nearly half of the sample (48.6\%) had a secondary educational level. A large proportion of people (42.4\%) had a net income of more than $€ 1500$ (per month/per household). When using the proposed cut-off points, the GFI detected 245 frail cases (46.3\%). The TFI and the SPQ identified $40.2 \%$ and $59.1 \%$ of the population as frail, respectively. The mean GARS total score at T1 was 24.9 (range 18-72) and 24.2 at $\mathrm{T} 2$, indicating hardly any change in disability on a population level over a one year period. In comparison with participants that remained in the sample $(n=430)$, nonresponders on T2 $(n=74)$ had slightly more difficulties in performing activities of daily living, a lower educational background, and lower income. However, these non-responders were not significantly more frail.

Out of 430 older persons, 105 (24\%) experienced development of disability; they became dependent on at least on one (other) of the 18 GARS activities during follow-up ( $\geq 1$ new disability). 75 persons (17\%) were admitted to a hospital during follow-up. Fifteen persons had died, and three became nursing home residents.

The calculations of receiver operating characteristics showed that all three instruments perform best with their proposed cut off points (GFI, 4; TFI, 5; SPQ, 2). Values for area under curve for all three instruments at these cut off points related to all dependent variables (development of disability, mortality, and hospital admission) are between 0.54 and 0.67 .

Table 2 Distribution of development of disability, mortality and hospital admission according to frailty scores

\begin{tabular}{|c|c|c|c|c|c|c|}
\hline & \multicolumn{2}{|c|}{$\begin{array}{l}\text { Functional decline } \\
(\mathrm{N}=426)^{*}\end{array}$} & \multicolumn{2}{|l|}{$\begin{array}{l}\text { Mortality } \\
(\mathrm{N}=532)^{*}\end{array}$} & \multicolumn{2}{|c|}{$\begin{array}{l}\text { Hospital admission } \\
(\mathrm{N}=430)^{*}\end{array}$} \\
\hline & + & - & + & - & + & - \\
\hline GFI: + & 74 (38\%) & $120(62 \%)$ & 11 (4\%) & 234 (96\%) & $39(20 \%)$ & $159(80 \%)$ \\
\hline GFI: - & $30(13 \%)$ & $200(87 \%)$ & $4(1 \%)$ & 280 (99\%) & $36(16 \%)$ & 194 (84\%) \\
\hline TFI: + & 64 (41\%) & 93 (59\%) & $10(5 \%)$ & 201 (95\%) & $39(24 \%)$ & $122(76 \%)$ \\
\hline TFI: - & 39 (15\%) & $224(85 \%)$ & $5(2 \%)$ & 309 (98\%) & $35(13 \%)$ & $228(87 \%)$ \\
\hline SPQ: + & 85 (34\%) & $162(66 \%)$ & $10(3 \%)$ & $295(97 \%)$ & 55 (22\%) & 195 (78\%) \\
\hline SPQ: - & $18(11 \%)$ & 150 (89\%) & $4(2 \%)$ & 207 (98\%) & $17(10 \%)$ & 152 (90\%) \\
\hline
\end{tabular}

* Due to missings small differences between $\mathrm{n}$ and numbers of participant reported for each instrument can occur 
Based on the proposed cut off points, diagnostic values were calculated as shown in table 3. Compared to $71 \%$ for the GFI and $62 \%$ for the TFI, the SPQ has the highest sensitivity (83\%) regarding development of disabilities. Specificity is lowest for the SPQ (48\%). The positive predicted values of the GFI, TFI, and SPQ are up to $40 \%$ and all have high (at least $85 \%$ ) negative predicted values. Regarding mortality, sensitivity for all tools are about $70 \%$ and specificity between $41 \%$ and $61 \%$. Positive predicted values are very low, and negative predicted values all very high. Regarding hospital admission, SPQ scores the highest for sensitivity (76\%), compared to GFI $(52 \%)$ and TFI (53\%). In contrast, specificity is lower for SPQ (44\%) in comparison with GFI (55\%) and TFI (65\%).

The unadjusted odds ratios for elderly identified as frail to develop disability are for all three instruments significant and close to 4 (GFI, 4,11; TFI, 3.96 ;SPQ, 4.36). Adjusted odds ratios for disability are, as expected, lower but still significant for all instruments. The unadjusted odds ratios for mortality are only significant for GFI (3.29) and TFI (3.08), but their impact diminishes after adjusting for baseline characteristics and GARS T1 scores. Regarding hospital admission, only the unadjusted odds ratios of TFI and SPQ reach significance.

Table 3 Diagnostic values of the screening tools for development of disabilities, mortality and hospital admission

\begin{tabular}{|c|c|c|c|c|c|}
\hline & $\begin{array}{l}\text { Sensitivity } \\
(95 \% \text { C.I. })^{*}\end{array}$ & $\begin{array}{l}\text { Specificity } \\
(95 \% \text { C.I. })^{*}\end{array}$ & $\begin{array}{l}\text { Positive } \\
\text { predicted value } \\
(95 \% \text { C.I.)* }\end{array}$ & $\begin{array}{l}\text { Negative } \\
\text { predicted value } \\
(95 \% \text { C.I.)* }\end{array}$ & $\begin{array}{l}\text { Area under the } \\
\text { Curve (95\% C.I.) }\end{array}$ \\
\hline \multicolumn{6}{|c|}{ Development of Disabilities ( $n=426$ ) } \\
\hline GFI & $71(62-79)$ & $63(57-68)$ & $38(31-45)$ & $87(81-90)$ & $67(61-73)$ \\
\hline TFI & $62(52-71)$ & $71(65-76)$ & $40(33-49)$ & $85(80-89)$ & $66(60-72)$ \\
\hline SPQ & $83(74-89)$ & $48(42-54)$ & $34(29-41)$ & $89(83-93)$ & $65(60-71)$ \\
\hline \multicolumn{6}{|c|}{ Mortality $(n=532)$} \\
\hline GFI & 73 (44-91) & $54(50-58)$ & $4(2-8)$ & 98 (96-99) & $64(50-77)$ \\
\hline TFI & $67(39-87)$ & $61(56-65)$ & $5(2-8)$ & 98 (96-99) & $64(50-78)$ \\
\hline SPQ & $71(42-90)$ & $41(37-46)$ & $3(1-6)$ & $98(94-98)$ & $56(42-71)$ \\
\hline \multicolumn{6}{|c|}{ Hospital admission ( $n=430$ ) } \\
\hline GFI & $52(40-64)$ & $55(50-60)$ & $20(15-26)$ & $84(79-89)$ & $54(46-61)$ \\
\hline TFI & $53(41-64)$ & $65(60-70)$ & $24(18-32)$ & $87(82-90)$ & $60(52-67)$ \\
\hline SPQ & $76(65-85)$ & 44 (39-49) & $22(17-28)$ & $90(84,94)$ & $60(53-67)$ \\
\hline
\end{tabular}

* Presented in percentages 
Table 4 Odds ratios Unadjusted and Adjusted for sex, age, income, education, GARS (T1)

\begin{tabular}{lllllll}
\hline & \multicolumn{2}{l}{ Development of disabilities } & Mortality & \multicolumn{3}{c}{ Hospital admission } \\
& OR & OR & OR & OR & OR & OR \\
& $\begin{array}{l}\text { Unadjusted } \\
(95 \% \text { C.I.) }\end{array}$ & $\begin{array}{l}\text { Adjusted } \\
(95 \% \text { C.I. })\end{array}$ & $\begin{array}{l}\text { Unadjusted } \\
(95 \% \text { C.I. })\end{array}$ & $\begin{array}{l}\text { Adjusted } \\
(95 \% \text { C.I. })\end{array}$ & $\begin{array}{l}\text { Unadjusted } \\
(95 \% \text { C.I. })\end{array}$ & $\begin{array}{l}\text { Adjusted } \\
(95 \% \text { C.I.) }\end{array}$ \\
\hline GFI & $4.11 *$ & $2.62 *$ & $3.29 *$ & 1.35 & 1.40 & 1.33 \\
& $(2.54-6.65)$ & $(1.48-4.64)$ & $(1.03-10.47)(0.32-5.76)$ & $(0.84-2.33)$ & $(0.73-2.41)$ \\
TFI & $3.96 *$ & $2.00 *$ & $3.08 *$ & 1.05 & $2.08 *$ & $2.59 *$ \\
& $(2.48-6.30)$ & $(1.18-3.57)$ & $(1.04-9.13)$ & $(0.24-4.60)$ & $(1.26-3.46)$ & $(1.36-4.90)$ \\
SPQ & $4.37 *$ & $2.49 *$ & 1.75 & 0.92 & $2.56 *$ & $2.42 *$ \\
& $(2.51-7.61)$ & $(1.35-4.61)$ & $(0.54-5.67)$ & $(0.20-4.33)$ & $(1.40-4.67)$ & $(1.27-4.62)$ \\
\hline
\end{tabular}

*Significant at $p \leq 0,05$

\section{Discussion}

The aim of this study was to compare the predictive values of three short postal screening instruments for identifying community dwelling frail older persons: the Groningen Frailty Indicator (GFI), the Tilburg Frailty Indicator (TFI) and the Sherbrooke Postal Questionnaire (SPQ).

All instruments perform best at their proposed cut off points. However, the associated AUC values, between 0.54 and 0.67 , indicate poor performance regarding prediction of any of the dependent variables (development of disability, mortality, and hospital admission). Despite high prevalences of frailty (between 40 and 60\%), the positive predicted values of the tools are low. The adjusted odds ratios show that those identified as frail have more than twice the risk (GFI, 2.62; TFI, 2.00; SPQ, $2,49)$ for developing disabilities within one year compared to the non-frail group; those identified as frail by the TFI and SPQ have more than twice the risk of being admitted to a hospital.

This is the first time that these three instruments are compared in one study for their predictive values. The postal procedure proved to be feasible with high response rates. A limitation of our study can be that, by dichotomizing development of disabilities, we might have missed more subtle changes in performance of activities. However, from a clinical perspective, a change from independent to dependent seems more important. Previous studies into frailty used a similar approach for the development of disabilities. ${ }^{17,18}$ One could argue that the follow-up period of one year is too short to monitor relevant adverse outcomes. However, in our study $24 \%$ of older persons did develop disabilities over a one-year period, and from a GP perspective, one year seems a reasonable timeframe for pro-active elderly care. Finally, the SPQ was not used according to protocol ${ }^{5}$, as non-responders were excluded 
from analyses. If we had considered non-responders also at risk, this would have resulted at $\mathrm{T} 1$ in a frailty prevalence estimate of $67.0 \%$ instead of $59.1 \%$.

Our diagnostic values of the SPQ for development of disabilities are comparable with those Hébert et al. ${ }^{5}$ found among elderly persons over 74 (sensitivity $75 \%$ and specificity $52 \%$ ). Gobbens et al. ${ }^{7}$ presented for the TFI a sensitivity of $84 \%$ and specificity of $76 \%$ for identifying frail elderly at risk for disability. However, this was based on a cross-sectional study design. As mentioned earlier, the adjusted odds ratios show that those identified as frail by the GFI, TFI, and SPQ have, more than twice the risk for developing disabilities within one year. Sarkisian et al. ${ }^{19}$ found in a cohort study that elders identified as frail with the CHS frailty index, as proposed by Fried et al. ${ }^{20}$, had a age-adjusted odds ratio of 4.4 for disability over a 4 year period. Ensrud et al. ${ }^{18}$ found, in a prospective cohort study for women $(\geq 69)$ identified frail with the CHS frailty index, a higher age adjusted risk (OR 2.2-2.8) for disability ( $\geq 1$ new IADL disability) over a period of 4 and a half years. Differences in estimated risks between those and our study may be attributed for a large part to variation in follow-up periods.

What are the implications for practice? Our previous study ${ }^{21}$ showed that extensive assessment after screening is necessary before intervention as the scalability of the instruments is poor. Such assessment is further recommended, as the high prevalence of frailty may point to the possibility that a substantial proportion of these elderly is pre-frail. In a two-step approach, the sensitivity will be the most relevant criterion. In that perspective, the SPQ scores best, followed by the GFI. The SPQ has the highest sensitivity (83\%) for development of disabilities; though with a positive predictive value of $34 \%$, a large proportion of older persons that do not develop disabilities are identified. General practitioners will regard the large number of false positives as burdensome, as too many older persons have to go through an unnecessary extensive assessment. Despite that predictive values for the $\mathrm{GFI}, \mathrm{TFI}$, and SPQ are not optimal, these instruments do have potential for use in community programs. Further research on these and other instruments is needed to improve the targeting of frail older persons.

\section{Acknowledgements}

The authors would like to thank: the elderly people who participated in this study, the general practitioners and their staff, the Centre for Data and Information Management at Maastricht University (MEMIC), Silke Metzelthin MSc. and Melanie Kleynen MSc. for their assistance in the data collection. We thank Dr. Marieke Spreeuwenberg for statistical advice. This study was funded by Stichting Innovatie Alliantie and Zuyd University of Applied Sciences. 


\section{References}

1. Bergman H, Ferrucci L, Guralnik J, Hogan DB et al. Frailty: an emerging research and clinical paradigm--issues and controversies. J Gerontol A Biol Sci Med Sci 2007;62(7):731-7.

2. Walston J, Hadley EC, Ferrucci L, Guralnik JM et al. Research agenda for frailty in older adults: toward a better understanding of physiology and etiology: summary from the American Geriatrics Society/National Institute on Aging Research Conference on Frailty in Older Adults. J Am Geriatr Soc 2006;54(6):991-1001.

3. Abellan van Kan G, Rolland YM, Morley JE et al. Frailty: toward a clinical definition. J Am Med Dir Assoc 2008;9(2):71-2.

4. De Lepeleire J, Degryse J, Illiffe S, Mann E et al. Family physicians need easy instruments for frailty. Age Ageing 2008;37(4):484; author reply -5 .

5. Hebert R, Bravo G, Korner-Bitensky N, Voyer L. Predictive validity of a postal questionnaire for screening community-dwelling elderly individuals at risk of functional decline. Age Ageing 1996;25(2):159-67.

6. Steverink N, Slaets JPJ, Schuurmans H, Lis van M. Measuring Frailty. Development and testing of the Groningen Frailty Indicator (GFI). Gerontologist 2001;41:236-7.

7. Gobbens RJ, van Assen MA, Luijkx KG, Wijnen-Sponselee MT et al. The Tilburg Frailty Indicator: psychometric properties. J Am Med Dir Assoc 2010;11(5):344-55.

8. Roberts HC, Hemsley ZM, Thomas G, Meakins $P$ et al. Nurse-led implementation of the single assessment process in primary care: a descriptive feasibility study. Age Ageing 2006;35(4):394-8.

9. Walker L, Jamrozik K, Wingfield D. The Sherbrooke Questionnaire predicts use of emergency services. Age Ageing 2005;34(3):233-7.

10. Roberts H, Hemsley Z, Thomas G, Aihie Sayer A et al. Can the Sherbrooke postal questionnaire predict who will require comprehensive assessment in the single assessment process? British Geriatrics Society, Birmingham, UK, Spring Meeting 2005,13-15 Apr 2005.

11. Metzelthin SF, Daniels R, van Rossum E, de Witte $L$ et al. The psychometric properties of three selfreport screening instruments for identifying frail older people living in the community BMC Public Health 2010;10:176.

12. Kempen GI, Miedema I, Ormel J, Molenaar W. The assessment of disability with the Groningen Activity Restriction Scale. Conceptual framework and psychometric properties. Soc Sci Med 1996;43(11):1601-10.

13. Steinbusch C. Screening of frail elderly in the community. The feasibility and psychometric properties of three instruments [MSc thesis]. Maastricht: Maastricht; 2008.

14. Schuurmans H, Steverink N, Lindenberg S, Frieswijk N et al. Old or frail: what tells us more? J Gerontol A Biol Sci Med Sci 2004;59(9):M962-5.

15. Kempen GIJM, Doeglas DM, Suurmeijer TPBM. The asessment of (I)ADL with the Groningen Activity Restriction Scale-a manual (in Dutch). Groningen, The Netherlands: Nothern Centre for Health Care Research, University of Groningen; 1993.

16. Fox-Wasylyshyn SM, El-Masri, M.M. Focus on Research Methods Handling Missing Data in SelfReport Measures. Research in Nursing \& Health 2005;28 488-95.

17. Hoogerduijn JG, Schuurmans MJ, Korevaar JC, Buurman BM et al. Identification of older hospitalised patients at risk for functional decline, a study to compare the predictive values of three screening instruments. J Clin Nurs 2010;19(9-10):1219-25.

18. Ensrud KE, Ewing SK, Taylor BC, Fink HA et al. Comparison of 2 frailty indexes for prediction of falls, disability, fractures, and death in older women. Arch Intern Med 2008;168(4):382-9.

19. Sarkisian CA, Gruenewald TL, John Boscardin W, Seeman TE. Preliminary evidence for subdimensions of geriatric frailty: the MacArthur study of successful aging. J Am Geriatr Soc 2008;56(12):2292-7.

20. Fried LP, Tangen CM, Walston J, Newman $A B$ et al. Frailty in older adults: evidence for a phenotype. $J$ Gerontol A Biol Sci Med Sci 2001;56(3):M146-56. 
CHAPTER 4

21. Metzelthin SF, Daniels R, van Rossum E, de Witte LP et al. Psychometrische eigenschappen van drie screeningsinstrumenten voor kwetsbaarheid bij thuiswonende ouderen. Submitted. 


\section{CHAPTER 5}

\section{Interventions to prevent disability in frail community-dwelling elderly: a systematic review}

This chapter was published as:

Daniels R, van Rossum E, de Witte L, Kempen GIJM, van den Heuvel W. Interventions to prevent disability in frail community-dwelling elderly: a systematic review. BMC Health Services Research 2008;8:278 


\section{Abstract}

\section{Background}

There is an interest for intervention studies aiming at the prevention of disability in community-dwelling physically frail older persons, though an overview on their content, methodological quality and effectiveness is lacking.

Methods: A search for clinical trials involved databases PubMed, CINAHL and Cochrane Central Register of Controlled Trials and manually hand searching. Trials that included community-dwelling frail older persons based on physical frailty indicators and used disability measures for outcome evaluation were included. The selection of papers and data-extraction was performed by two independent reviewers. Out of 4602 titles, 10 papers remained that met the inclusion criteria. Of these, 9 were of sufficient methodological quality and concerned 2 nutritional interventions and 8 physical exercise interventions.

Results: No evidence was found for the effect of nutritional interventions on disability measures. The physical exercise interventions involved 2 single-component programs focusing on lower extremity strength and 6 multi-component programs addressing a variety of physical parameters. Out of 8 physical exercise interventions, three reported positive outcomes for disability. There was no evidence for the effect of single lower extremity strength training on disability. Differences between the multi-component interventions in e.g. individualization, duration, intensity and setting hamper the interpretation of the elements that consistently produced successful outcomes.

Conclusions: There is an indication that relatively long-lasting and high-intensive multicomponent exercise programs have a positive effect on ADL and IADL disability for community-living moderate physically frail older persons. Future research into disability prevention in physically frail older persons could be directed to more individualized and comprehensive programs. 


\section{Background}

Frail elderly people are at much higher risk for falls, infections, disabilities, hospitalization, institutionalization, and death, compared with their age-matched non-frail counterparts. $^{1-3}$ In scenarios that predict future health service delivery in the Western world, the rapid increase in frail elderly is seen as one of the major challenges of health care. ${ }^{4-6}$ There is an increasing interest for frail elderly being particularly vulnerable for developing disabilities. ${ }^{7-9}$ As disability is closely related to medical spending, it is believed that prevention of disability can lead to reduced health care costs. ${ }^{10}$ Current literature supports the notion of frailty as a pathway to disability that is not a direct result of chronic disease, but instead is associated with agerelated loss of physical condition and reserve. ${ }^{11,12}$ This viewpoint on frailty points to opportunities for interventions aimed at either delaying the onset of frailty or reducing its adverse outcomes. ${ }^{1,4}$

A widely accepted definition and clear criteria for frailty are lacking. ${ }^{1,5,13}$ Markle-Reid and Brown ${ }^{5}$ reported substantial disagreement in the literature how frailty is defined and measured. Based on recent studies into risk-factors for adverse outcomes in frail elderly there appears to be a growing consensus for acknowledging physical frailty as a construct that can be identified by frailty components. ${ }^{14}$ Interventions for physical frailty stem from the idea that the causal pathway towards frailty is a negative spiral in which inflammation, neuroendocrine deregulation and sarcopenia play a role implying that interventions can be targeted at physical frailty independent of specific diseases. ${ }^{15}$ The Interventions on Frailty Working Group ${ }^{14}$ recommended the development and testing of preventive interventions for physically frail elderly based on any of the following screening criteria: mobility, strength, balance, motor processing, nutrition, endurance or physical activity. Over the last decade, several intervention studies aiming at the prevention of disability in the elderly using physical frailty indicators as inclusion criteria have been reported. An overview of the effectiveness and content of these interventions is, however, not available. This systematic review was conducted to assess the content, the methodological quality and the effectiveness of intervention studies for the prevention of disability in community-dwelling physically frail elderly.

\section{Methods}

\section{Search strategy}

On May 162007 databases PubMed, the Cochrane Central Register of Controlled Trials (CENTRAL) and CINAHL were searched for randomized- and controlled clinical 
trials by using "frail*", "vulnerable", "at risk", "high risk", "low functioning", and the MESH terms "chronic disease" and "disabled persons" in combination with the MESH term "aged". Search terms for outcomes focused on disability measures and included terms like "disabil*", "functional decline", "functional capabilit*", "functional performance", "independen*" and MESH terms "activities of daily living", "quality of life" and "well being". To restrict the search to interventions that targeted community-dwelling elderly terms like "home*", "in-home*", "communit*", "independent living" and MESH term "primary care" were added. Additionally studies were searched by hand-searching reference lists from relevant papers. The search was restricted to articles in English, Dutch and German. There was no restriction for type of intervention or year of publication.

\section{Selection criteria}

Clinical trials where community-dwelling frail elderly were the target group were included. Studies had to include frail elderly based on at least one of the physical frailty indicators as described by Ferrucci et al. ${ }^{14}$ Table 1 gives an overview of these indicators. Studies that, for instance, equalized frailty solely on the basis of presence of disabilities, chronic illness, the eligibility of care or discharge from hospital were excluded. As interventions in frail elderly focusing on disability prevention was the aim of this study, the presence of outcome measurement 'disability' was another criteria for inclusion. Disability was defined as experienced difficulty in performing activities in any domain of life. ${ }^{16}$ Avlund ${ }^{17}$ found that most current studies of disability among older persons focus on the ability to carry out the activities of daily living. The need to focus on activities of daily living as a fundamental outcome is well justified as persons who are disabled in activities of daily living function can not successfully live alone. ${ }^{18}$ In this review only studies reporting about measurements on Activities of Daily Living (ADL) or Instrumental Activities of Daily Living (IADL) were included. 
Table 1 Physical Frailty Indicators

\begin{tabular}{|c|c|}
\hline Indicators* & Possible measures $^{+}$ \\
\hline Mobility & Gait speed \\
\hline \multirow[t]{3}{*}{ Strength } & Grip strength \\
\hline & Chair rise \\
\hline & Knee extensor strength \\
\hline \multirow[t]{3}{*}{ Endurance } & Lack of energy \\
\hline & Tiredness \\
\hline & Oxygen-uptake \\
\hline \multirow[t]{4}{*}{ Nutrition } & Under-nutrition (decreased food intake) \\
\hline & Weight loss \\
\hline & Body Mass Index \\
\hline & Obesity \\
\hline Physical inactivity & $\begin{array}{l}\text { Frequency and duration of walking and bicycling in the previous week and the } \\
\text { average amount of time spent monthly on hobbies, gardening, odd jobs, and } \\
\text { sports }\end{array}$ \\
\hline \multirow[t]{4}{*}{ Balance } & Items from Berg Balance Scale like \\
\hline & Sitting to standing \\
\hline & Standing to sitting \\
\hline & Standing unsupported \\
\hline \multirow[t]{3}{*}{ Motor processing } & Coordination \\
\hline & Movement planning \\
\hline & Movement speed \\
\hline
\end{tabular}

* As mentioned in Ferrucci et al. ${ }^{14}$

${ }^{+}$As reported in frailty literature

\section{Data extraction and analysis}

A first selection of relevant studies was made on title-level using a conservative approach, meaning that in case of doubt an article would always be screened on abstract-level. The second (abstract-level) and third selection phase (full-text level) were independently undertaken by two reviewers (RD and EvR) scoring 'relevant', 'doubt' or 'irrelevant' on forms. In case of inconsistencies, the reviewers discussed the scoring. Consensus on 'irrelevant' led to the exclusion of an article. On one occasion the reviewers asked for the involvement of a third party (LdW) in order to reach consensus. The same reviewers also performed independently the quality assessment of included studies as well as the data extraction. Inconsistencies in scoring between reviewers was discussed until consensus was reached. As the included trials all turned out to be randomized controlled trials the methodological quality was assessed using an adaptation of the Cochrane Back Review Group list of criteria (Table 2). ${ }^{19}$ Three items were disregarded, because of their use as an inclusion criterion (relevance of outcome measure) or their low applicability to the 
evaluated interventions (blinding of participant and blinding of care provider). The criteria list comprised five descriptive, two statistical and nine validity items. Each item was scored "+" if the criterion was fulfilled, "-" if the criterion was not fulfilled, and "?" if the information was not provided or was unclear. Scores on validity items ranged from 0 to 9 per trial. Trials which fulfilled over half of the validity items were considered to be of "sufficient methodological quality". In addition, general characteristics of the studies and outcomes of the trials were extracted. All data were analyzed qualitatively. Pooling of data was considered inappropriate due to the heterogeneity between trials regarding measurement instruments and intervention characteristics.

Table 2 Extracted data from the selected full papers

\section{Methodological Quality of Trials (Criteria List by Cochrane Back Review Group)}

\section{Descriptive Items}

1. Were eligibility criteria clearly specified?

2.Were index and control interventions explicitly described?

3. Was described whether adverse effects had or had not occurred?

4. Was a short-term follow-up measurement (directly after the intervention) of disability performed?

5. Was a long-term follow-up measurement ( $>6$ months after the intervention) of disability performed?

\section{Statistical Items}

6. Was the sample size for each group described?

7. Were point estimates and measures of variability presented for disability?

Internal Validity Items

8. Was a method of randomization used?

9. Was treatment allocation concealed?

10. Were groups similar at baseline regarding the most important prognostic indicators?

11. Were co-interventions avoided or comparable?

12. Was the compliance acceptable in all groups?

13. Was the outcome assessor blinded to the intervention?

14. Was the withdrawal/dropout rate acceptable (maximum of $20 \%$ for short-term follow-up and $30 \%$ for long-term follow-up)?

15. Was timing of the outcome assessment in both groups comparable?

16. Did the analysis include an intention-to-treat analysis?

\section{Results of the Trial}

Disability outcome measure

Frailty components measures

Baseline and follow-up details: number of participants, time of follow-up and results of the analyses

\section{Results}

Four thousand six hundred and two titles were identified in the literature search. After screening the titles, 127 studies were considered relevant for further screen- 
ing on abstract-level. Of these, another 69 studies were excluded, because of not meeting the inclusion criteria (figure 1). In the next phase, the screening of 58 fulltext articles resulted in the exclusion of 48 studies. Thirty-eight trials were excluded for not meeting the criteria for population characteristics, 5 for not meeting the criteria for the outcome measure (disability) and 5 for not meeting both criteria. There was a 0.70 and 0.75 (Kappa value) agreement between the reviewers during respectively screening of abstracts and screening of full-text articles. Results of the methodological quality assessment of the 10 included trials are shown in table 3 . The observed total validity score ranged from 3 to 7 . One trial ${ }^{20}$ did not fulfill over half of the criteria $(\geq 5)$ and was considered to be of insufficient methodological quality. Most papers did not provide sufficient information on whether the treatment allocation was concealed and on whether co-interventions were avoided or comparable. Concerning the descriptive items, 9 out of the 10 trials did not perform a long-term follow-up measurement ( $\geq 6$ months after the intervention). Six trials did not report about the occurrence of adverse effects.

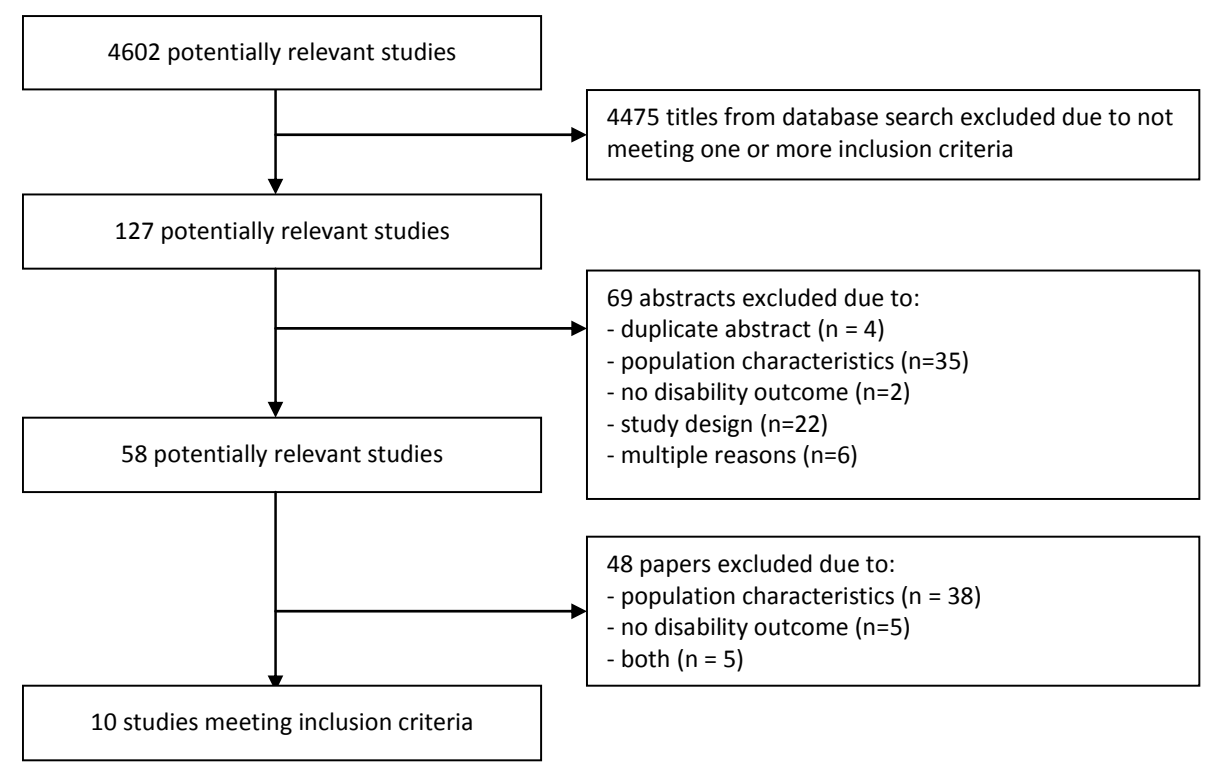

Figure 1 Progress of Search for Relevant Trials

Table 4 provides general characteristics of all 10 trials. From here the results of the 9 trials with sufficient methodological quality will be presented. The number of participants in the trials varied from 46 to 188. All studies were published between 1998 and 2005, showing the relative new trend in using frailty indicators explicitly as inclusion criteria. Disability was not the primary outcome measure for all studies. In the 9 trials the experimental interventions were nutritional interventions ${ }^{21,22}$ or physical exercise interventions ${ }^{21,23-29}$. The study by Chin A Paw ${ }^{21}$ followed a factorial 
design evaluating both a nutritional and a physical exercise program. The nutritional interventions in the selected studies were focused on macronutrient status ${ }^{22}$ or micronutrient status ${ }^{21}$. The physical exercise interventions were single-component focusing purely on lower extremity strength ${ }^{23,27}$ or multi-component addressing a variety of physical parameters as endurance, flexibility, balance and strength ${ }^{21,24-}$ $26,28,29$.

All interventions can be regarded as standard treatment (as in contrast to tailormade treatment) and focused on the physical condition of the participants, except for one ${ }^{26}$ that individualized treatment based on the outcomes of an extensive assessment and focused also on environmental conditions. The interventions lasted from 10 weeks to 18 months. The two longest programs ${ }^{25,26}$ intended to encourage participants to undertake home exercising independently and provided only monthly phone calls in the last 6 months. In this review additional support by telephone was considered a part of the intervention. The inclusion criteria used varied from rising from a chair, to descending stairs, knee extensor strength, oxygenuptake, physical inactivity, involuntary weight loss, low BMI, dietary assessment, gait test, balance test and mobility problems (see also inclusion criteria in table 4). Although all studies used measurements on ADL or IADL, not all mentioned disability as an outcome measure; some used other terms like functional decline ${ }^{26}$ or reduced functional ability ${ }^{24}$. The mean age of the populations in the included trials ranged from 76 to 83 .

The main results of the trials are presented in table 4 . All trials (except for one ${ }^{28}$ ) reported statistically significant positive changes on physical measures e.g. weight gain, strength, mobility, oxygen-uptake, physical fitness, physical activity and balance. Improvements on these physical measures did not necessarily lead to positive effects on disability outcomes, as only 3 out of nine trials reported significant differences in favor of the intervention group. ${ }^{24,26,29}$

Those three trials are multi-component physical exercise programs. In the study of Gill et al. ${ }^{26}$, the intervention group showed less functional decline at 12 months (from 2.3 to 2.7 on a scale with a range of 0 to 16) than the control group (from 2.8 to 4.2). Subgroup analysis revealed that these effects were mainly obtained for participants with moderate frailty but not for participants with severe frailty. Worm et al. ${ }^{24}$ reported for the intervention group a larger increase in functional ability (from 36.4 to 53.6 on a scale ranging from 0 to 100) compared to the control group (from 39.1 to 43.0). In the study of Binder et al. ${ }^{29}$ the intervention group reported less difficulty with ADL and IADL after the nine months program (from 26.6 to 30.4 on a scale ranging from 0 to 36) compared to the control group (from 26.6 to 27.0). Binder et al. ${ }^{29}$ specifically focused on moderate to mild frail elderly. 
Table 3 Methodological Quality of Included Trials*

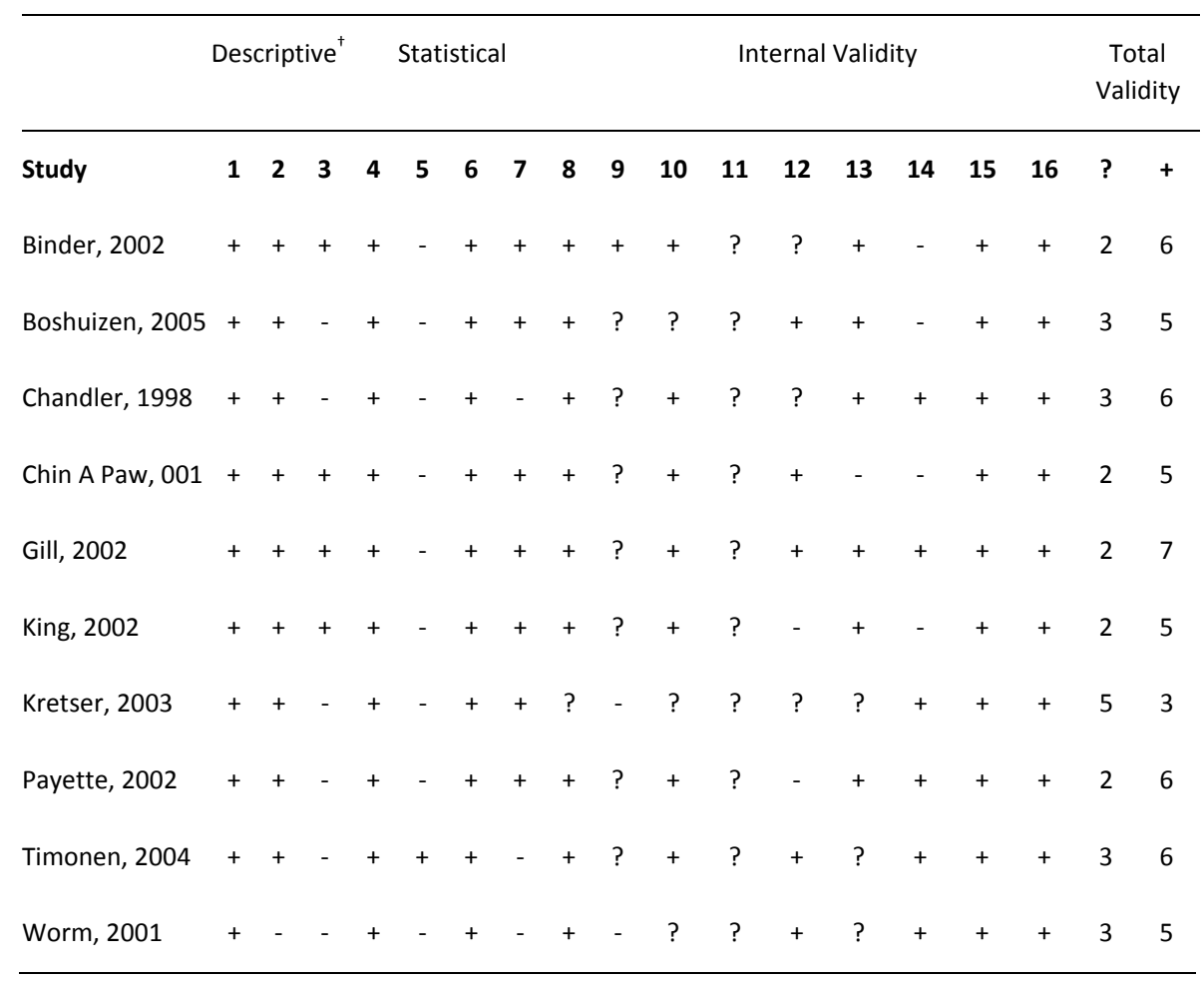

' + ' criterion fulfilled; '-' criterion not fulfilled; and '?' data not provided or unclear.

${ }^{\dagger}$ see table 1 for a detailed description of the items. 


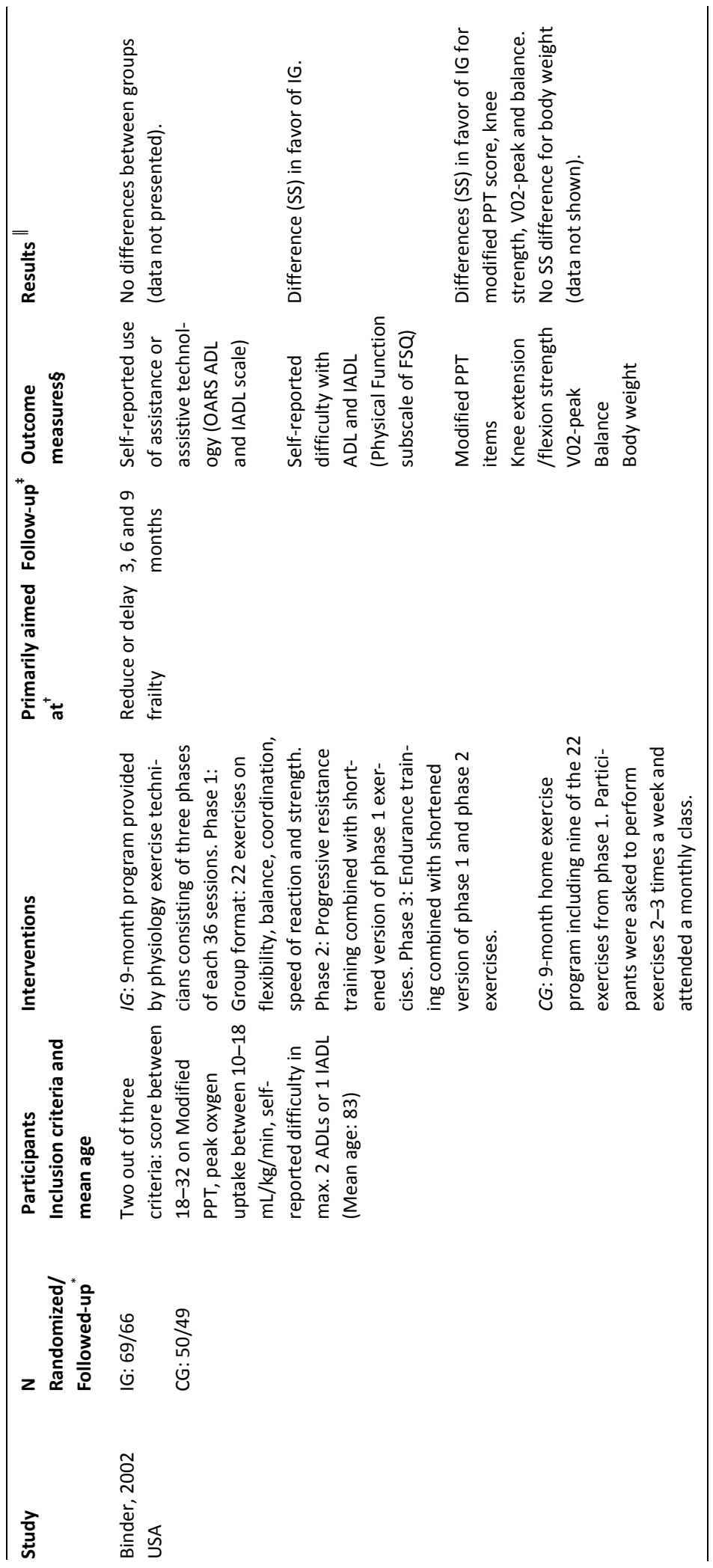




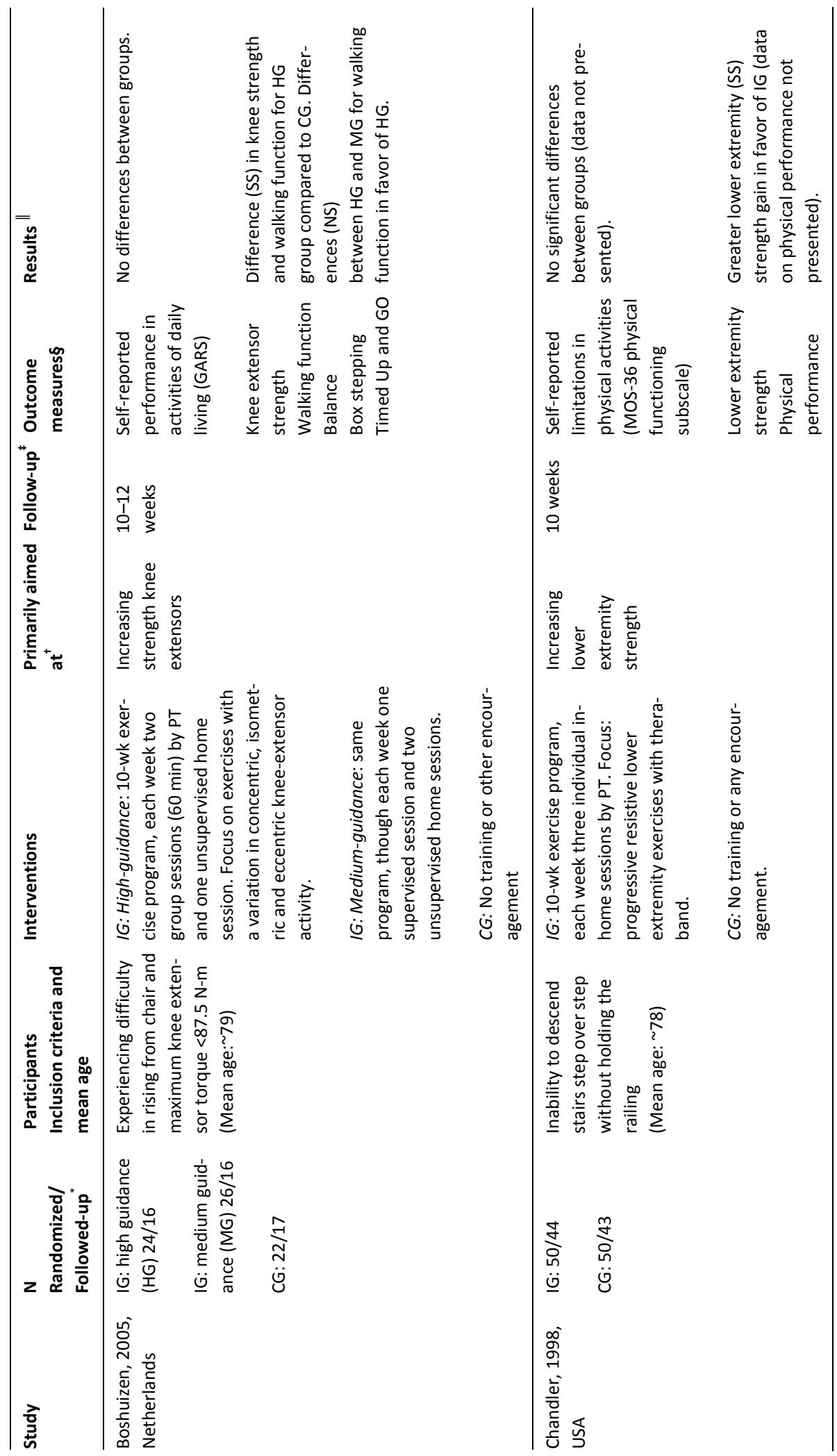




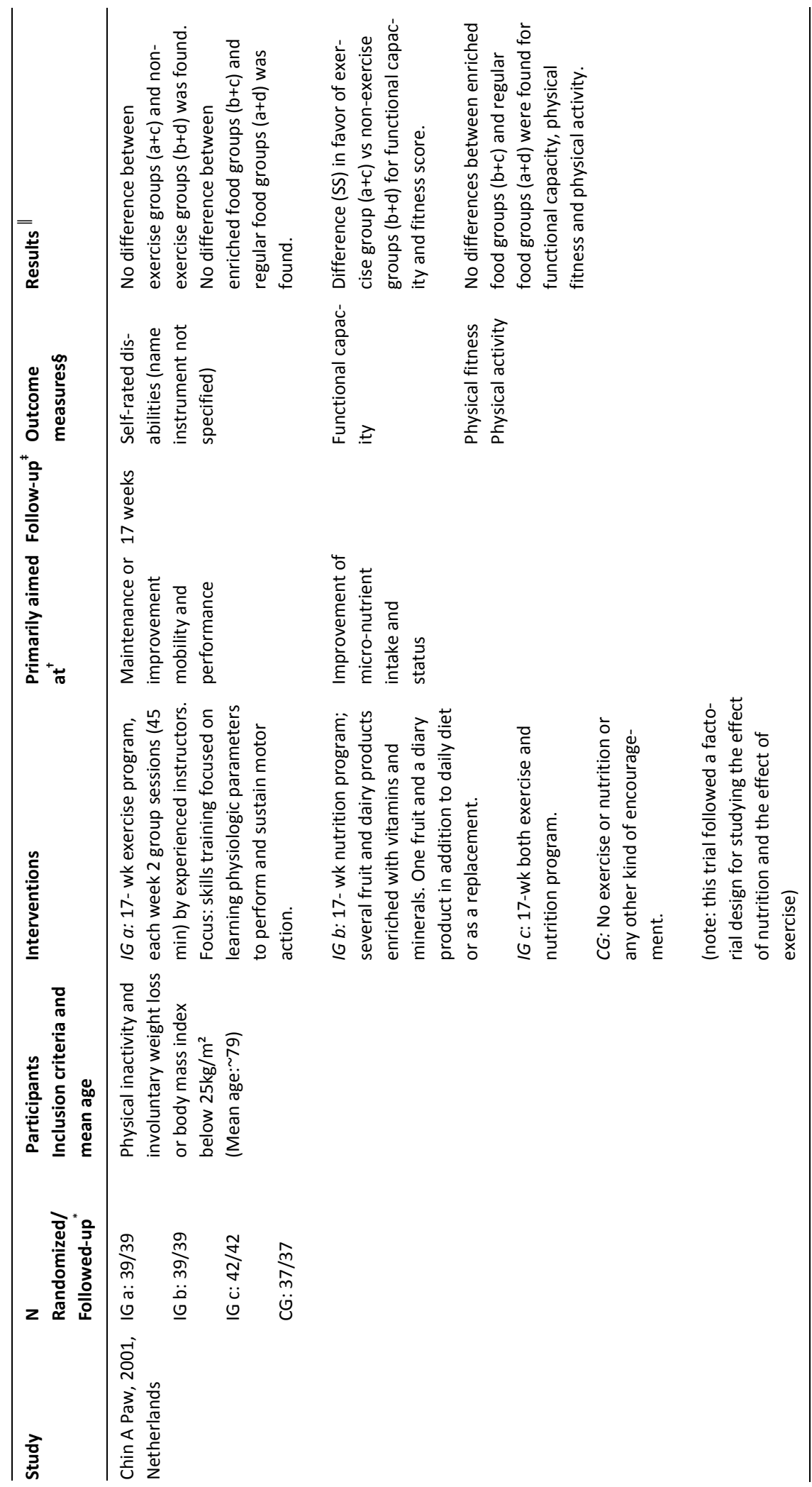


SYSTEMATIC REVIEW INTERVENTIONS PHYSICALLY FRAIL ELDERY

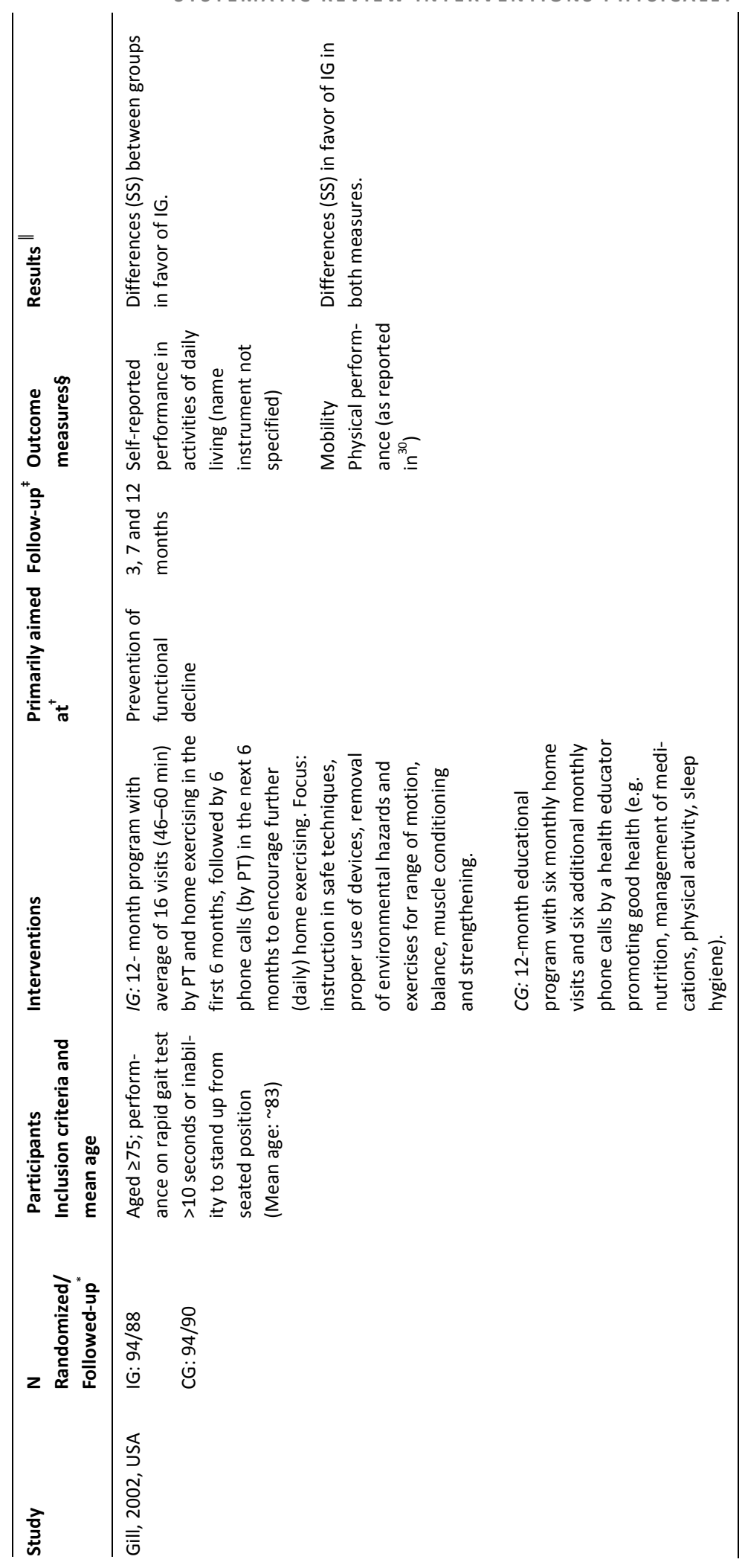




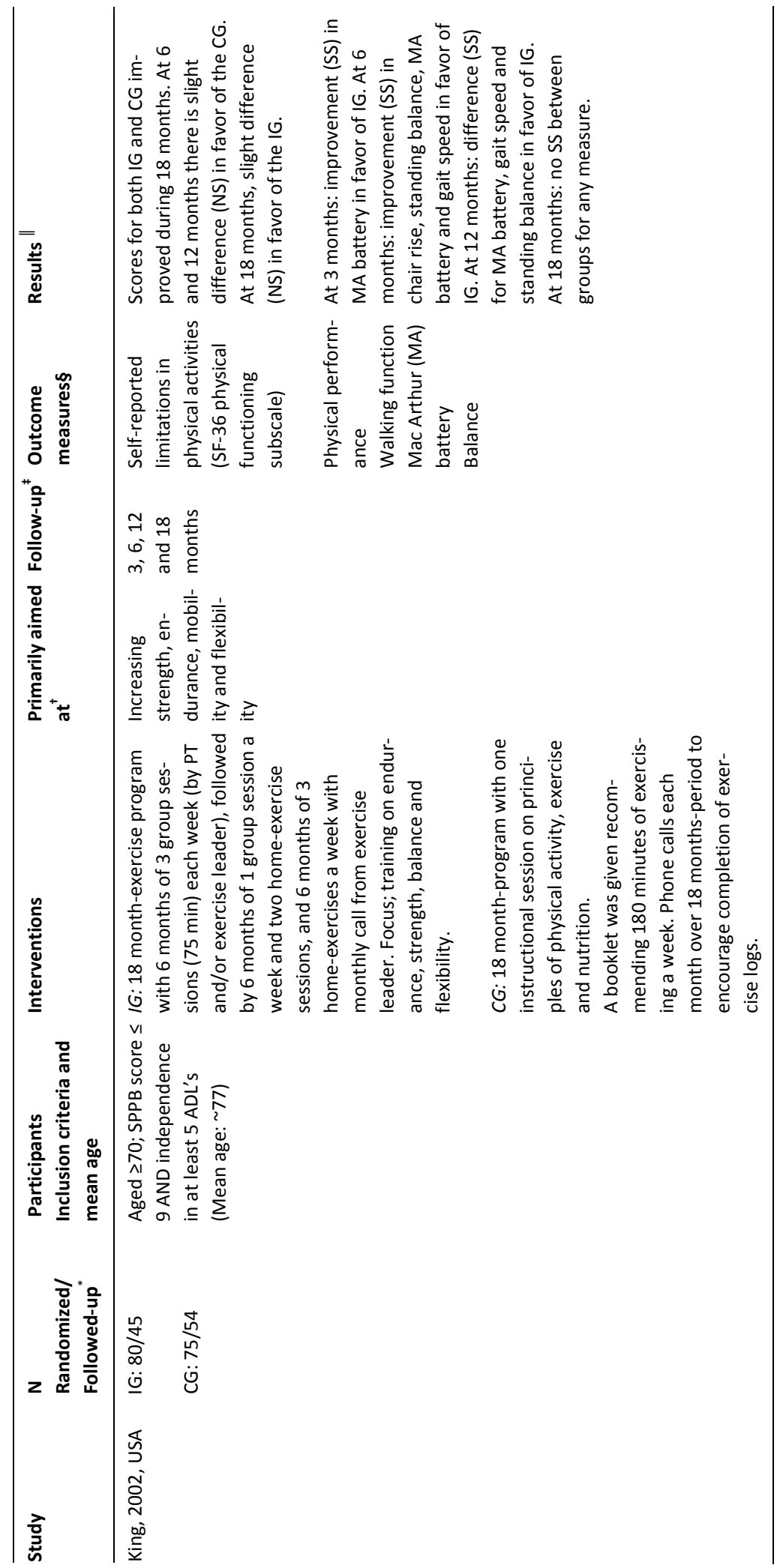




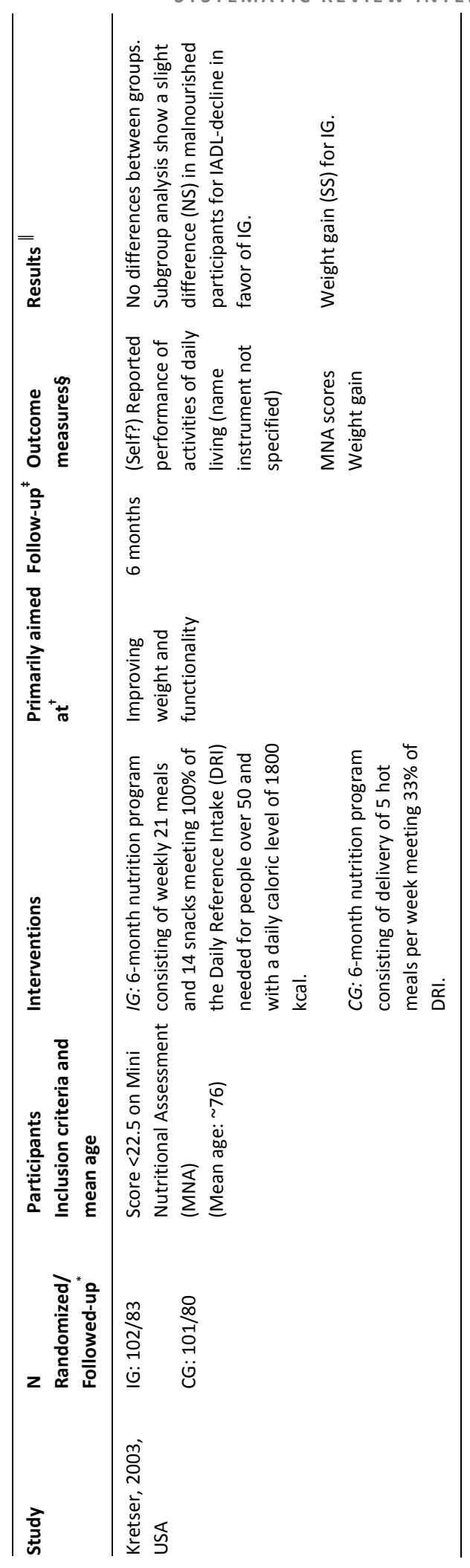




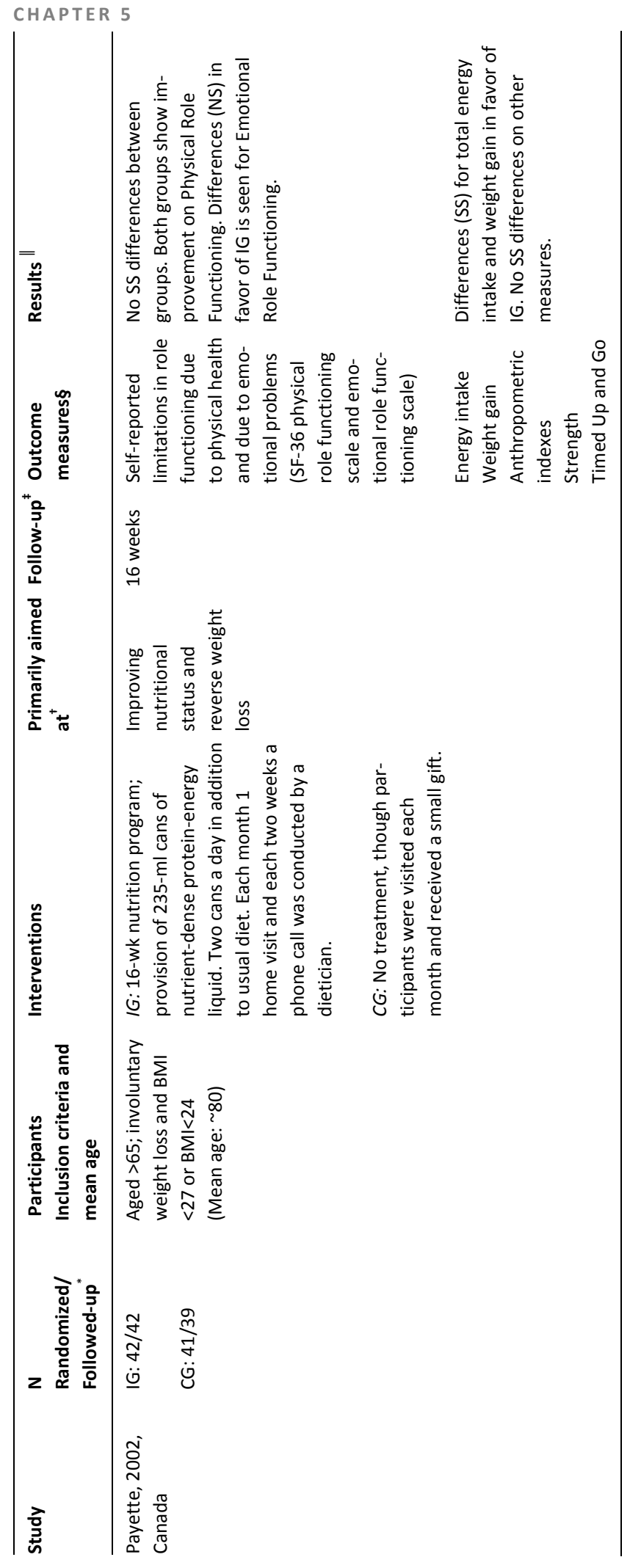




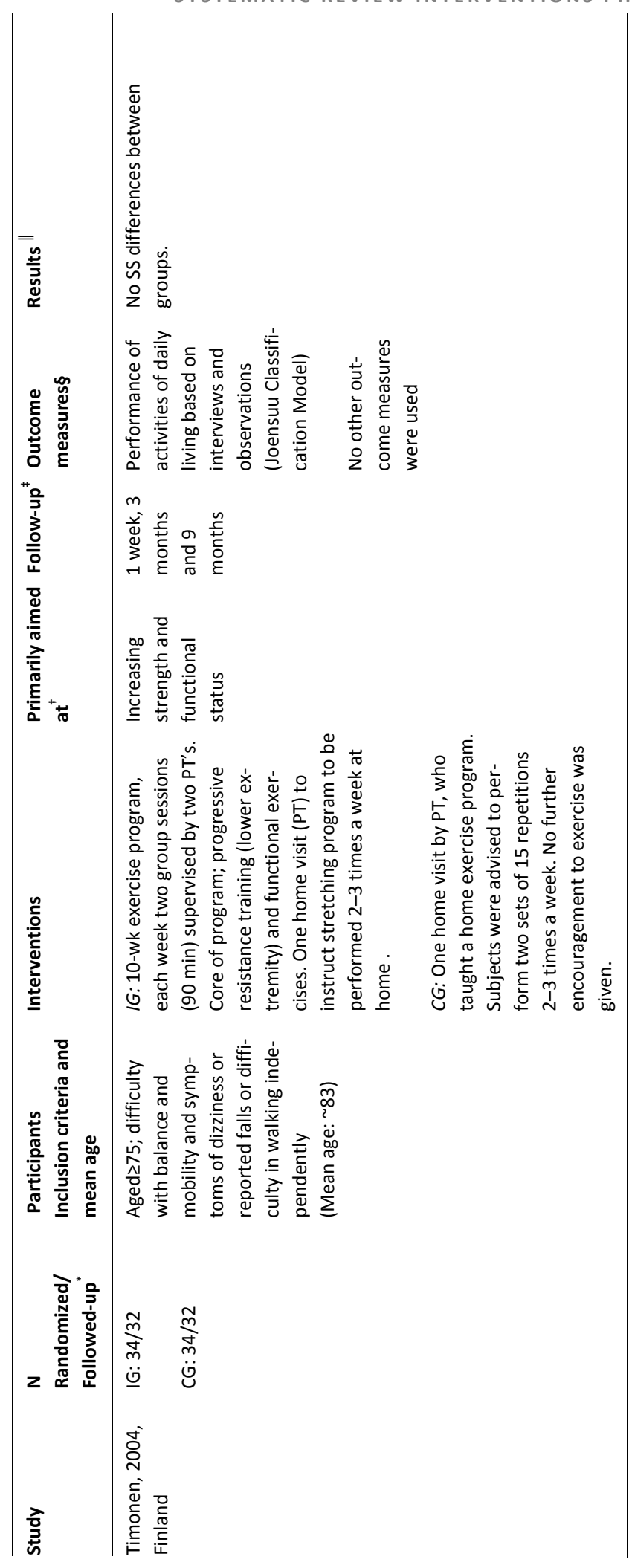




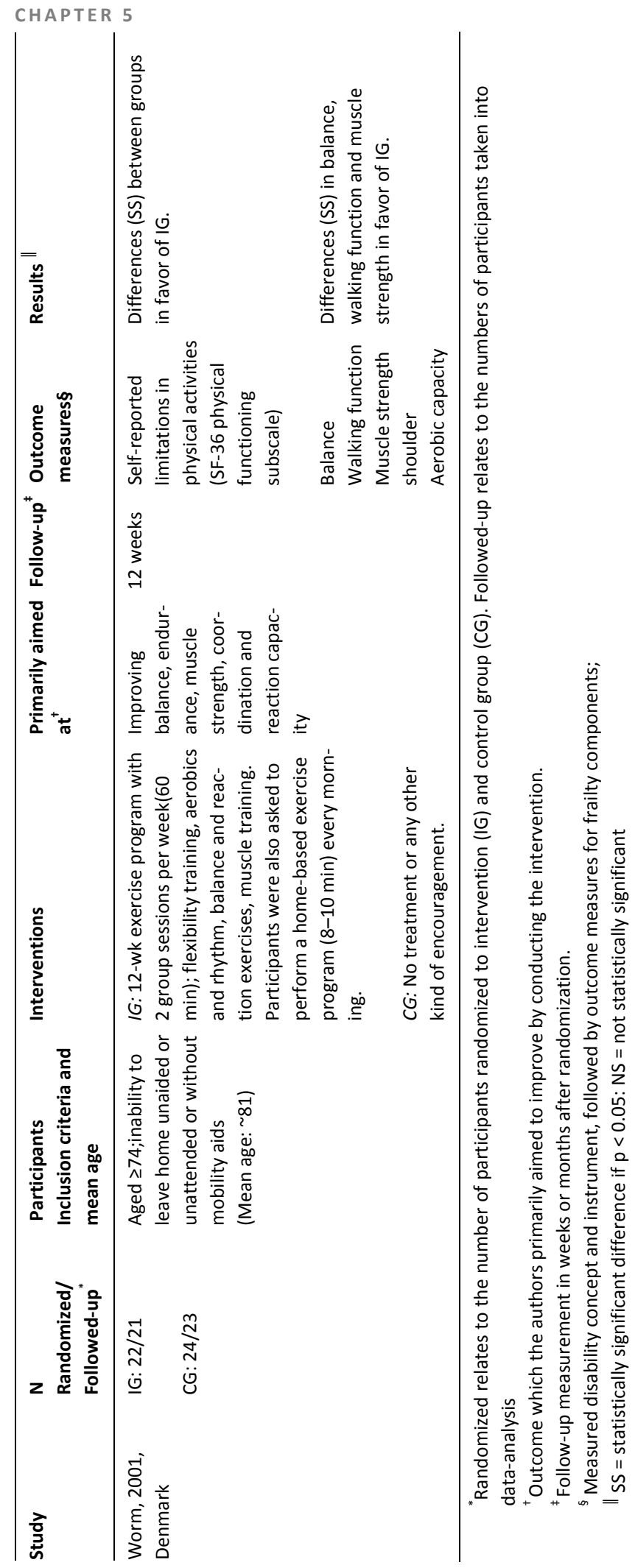




\section{Discussion}

This review aims to provide an overview of the content, methodological quality and effectiveness of intervention studies that are directed to physically frail elderly. In order to perform this review a strict use of criteria for physical frailty and disability was needed. This meant that even recent trials that contribute to frailty intervention research $\left(e . g^{31}\right)$, however not meeting the inclusion criteria, were excluded. The use of the term frailty in the literature is relatively new. ${ }^{4}$ Although reference lists were checked, a limitation of this review is that 'older studies' were probably not identified as we searched with terms for frailty and synonyms. Another risk for publication bias is the selection of $\mathbf{4 6 0 2}$ studies on title-level that may have resulted in excluding relevant articles. Due to a lack of consensus in the literature about measures for detecting frail elderly the homogeneity of the target groups in this review may have been reduced. In interpreting the results of this review it should be taken in consideration that due to small sample sizes trials may have been underpowered to detect differences on the self-reported measures for disability.

Out of the 10 randomized controlled trials that evaluated interventions for physically frail community-living elderly on disability, 9 were considered to be of sufficient methodological quality. No consistent findings were found in these 9 trials regarding their effect on disability.

There is no evidence that nutritional interventions for frail elderly, despite an observed effect on total energy intake and weight gain ${ }^{22}$, result in positive effects on disability-level. Out of 8 physical exercise interventions three reported positive outcomes for disability. No evidence appeared that single lower extremity strengthtraining, despite the effect on strength ${ }^{23,27}$ and walking function ${ }^{27}$ has an effect on disability. However, lower extremity strength training as part of a multicomponent program may contribute to the effectiveness of these programs. Out of six trials that offer a multi-component physical exercise program focusing on endurance, flexibility, balance and strength, three studies ${ }^{24,26,29}$ reported statistically significant effects for the disability outcome. The interventions in the effective studies of Gill et al. $^{26}$ and Binder et al. ${ }^{29}$ are relatively long-lasting programs (respectively 12 and 9 months) with at least three exercising moments a week. The effective program of Worm et al. ${ }^{24}$ last 12 weeks and comprises 2 supervised sessions a week and daily home exercises for 8 to 10 minutes. Gill et al.'s trial ${ }^{26}$ has some specific features compared to the other trials: it is more individualized, it focuses on both the person and the environment and it provides supervised individual home-sessions followed by six months of non-supervised exercising.

The differences between the interventions hamper the interpretation of elements that consistently produced successful outcomes. Although malnutrition and 
physical frailty markers are considered strong indicators for functional decline in the elderly $^{32,33}$, the question is whether targeting underlying mechanisms of frailty can prevent or delay disability. The effects of single nutritional interventions on functional performance is an issue under discussion ${ }^{32}$. A review on protein and energy supplementation in malnourished elderly ${ }^{34}$ reported evidence for weight gain, but found no evidence for positive effects on functional performance. Our finding that physical exercise interventions for community-dwelling frail elderly have an effect on intermediate physical measures is supported by reviews ${ }^{35-37}$ focusing on physical exercising for elderly. These identified a lack of evidence for the effect of physical exercise on disability, suggesting that prevention of disability needs to address a complex of physical, behavioral, environmental and social factors. This review on interventions for physically frail elderly shows some indication that long-lasting high-intensive exercise programs for moderate physically frail elderly can have an effect on disability outcomes. However, additional research is needed. As there are also indications that especially moderate frail elderly may benefit ${ }^{14,26}$ it is recommended to conduct subgroup analysis in effect studies for frail elderly.

\section{Conclusions}

A systematic review was conducted to assess the content, the methodological quality and the effectiveness of intervention studies for the prevention of (ADL/IADL) disability in community-dwelling physically frail older persons. There is no evidence that nutritional interventions for frail older persons, despite an observed effect on total energy intake and weight gain, result in positive effects on disability-level. No evidence appeared that single lower extremity strength-training, despite the effect on strength and walking function, has an effect on disability for physically frail older persons. There is some indication that long-lasting high-intensive exercise programs for moderate physically frail older persons can have an effect on disability outcomes.

\section{Acknowledgements}

We kindly thank dr. G.A.R. Zijlstra for her support in searching the database and mrs. A. Graat-Engelen for her support in retrieving publications. This review was funded by the Zuyd University of Applied Sciences. 


\section{References}

1. Aminzadeh F, Byszewski A, Dalziel WB, Wilson M, Deane N, Papahariss-Wright S. Effectiveness of outpatient geriatric assessment programs: exploring caregiver needs, goals, and outcomes. J Gerontol Nurs. 2005;31(12):19-25.

2. Storey E, Thomas RL. Understanding and ameliorating frailty in the elderly. Topics in Geriatric Rehabilitation. 2004;20(1):4-13.

3. Espinoza S, Walston JD. Frailty in older adults: insights and interventions. Cleve Clin J Med. 2005;72(12):1105-12.

4. Hooi WC, Bergman H. A review on models and perspectives on frailty in older persons. SGH Proceedings. 2005;14(2).

5. Markle-Reid M, Browne G. Conceptualizations of frailty in relation to older adults. J Adv Nurs. 2003;44(1):58-68.

6. Slaets JP. Vulnerability in the elderly: frailty. Med Clin North Am. 2006;90(4):593-601.

7. Hardy SE, Dubin JA, Holford TR, Gill TM. Transitions between states of disability and independence among older persons. Am J Epidemiol. 2005;161(6):575-84.

8. Boyd CM, Xue QL, Simpson CF, Guralnik JM, Fried LP. Frailty, hospitalization, and progression of disability in a cohort of disabled older women. Am J Med. 2005;118(11):1225-31.

9. Gill TM, Allore H, Holford TR, Guo Z. The development of insidious disability in activities of daily living among community-living older persons. Am J Med. 2004;117(7):484-91.

10. Cutler DM. Declining disability among the elderly. Health Affairs. 2001;20(6):11-27.

11. Fried LP, Tangen CM, Walston J, Newman AB, Hirsch C, Gottdiener J, et al. Frailty in older adults: evidence for a phenotype. J Gerontol A Biol Sci Med Sci. 2001;56(3):M146-56.

12. Fried LP, Ferrucci L, Darer J, Williamson JD, Anderson G. Untangling the concepts of disability, frailty, and comorbidity: implications for improved targeting and care. J Gerontol A Biol Sci Med Sci. 2004;59(3):255-63.

13. Rockwood K. What would make a definition of frailty successful? Age Ageing. 2005;34(5):432-4.

14. Ferrucci L, Guralnik JM, Studenski S, Fried LP, Cutler GB, Jr., Walston JD. Designing randomized, controlled trials aimed at preventing or delaying functional decline and disability in frail, older persons: a consensus report. J Am Geriatr Soc. 2004;52(4):625-34.

15. Walston J, Hadley EC, Ferrucci L, Guralnik JM, Newman AB, Studenski SA, et al. Research agenda for frailty in older adults: toward a better understanding of physiology and etiology: summary from the American Geriatrics Society/National Institute on Aging Research Conference on Frailty in Older Adults. J Am Geriatr Soc. 2006;54(6):991-1001.

16. Jette AM. Towards a common language for function, disability and health. Physical Therapy. 2006;86(5):726-34.

17. Avlund K. Disability in old age. Longitudinal population-based studies of the disablement process. Dan Med Bull. 2004;51(4):315-49.

18. Covinsky K. Aging, arthritis, and disability. Arthritis Rheum. 2006;55(2):175-6.

19. Tulder van MW, Cherkin DC, Berman B, Lao L, Koes BW. The effectiveness of acupuncture in the management of acute and chronic low back pain. A systematic review within the framework of the Cochrane Collaboration Back Review Group. Spine. 1999;24(11):1113-23.

20. Kretser AJ, Voss T, Kerr WW, Cavadini C, Friedmann J. Effects of two models of nutritional intervention on homebound older adults at nutritional risk. Journal of the American Dietetic Association. 2003;103(3):329-36.

21. Chin A Paw MJ, de Jong N, Schouten EG, Hiddink GJ, Kok FJ. Physical exercise and/or enriched foods for functional improvement in frail, independently living elderly: a randomized controlled trial. Arch Phys Med Rehabil. 2001;82(6):811-7.

22. Payette H, Boutier V, Coulombe C, Gray-Donald K. Benefits of nutritional supplementation in freeliving, frail, undernourished elderly people: a prospective randomized community trial. J Am Diet Assoc. 2002;102(8):1088-95. 
23. Chandler JM, Duncan PW, Kochersberger G, Studenski S. Is lower extremity strength gain associated with improvement in physical performance and disability in frail, community-dwelling elders? Arch Phys Med Rehabil. 1998;79(1):24-30.

24. Worm CH, Vad E, Puggaard L, Stovring H, Lauritsen J, Kragstrup J. Effects of a multicomponent exercise program on functional ability in community-dwelling frail older adults. Journal of the aging and physical activity. 2001;(9):414-24.

25. King MB, Whipple RH, Gruman CA, Judge JO, Schmidt JA, Wolfson LI. The Performance Enhancement Project: improving physical performance in older persons. Arch Phys Med Rehabil. 2002;83(8):10609.

26. Gill TM, Baker DI, Gottschalk M, Peduzzi PN, Allore H, Byers A. A program to prevent functional decline in physically frail, elderly persons who live at home. N Engl J Med. 2002;347(14):1068-74.

27. Boshuizen HC, Stemmerik L, Westhoff MH, Hopman-Rock M. The effects of physical therapists' guidance on improvement in a strength-training program for the frail elderly. J Aging Phys Act. 2005;13(1):5-22.

28. Timonen L, Rantanen T, Makinen E, Timonen TE, Tormakangas T, Sulkava R. Effects of a group-based exercise program on functional abilities in frail older women after hospital discharge. Aging Clin Exp Res. 2006;18(1):50-6.

29. Binder EF, Schechtman KB, Ehsani AA, Steger-May K, Brown M, Sinacore DR, et al. Effects of exercise training on frailty in community-dwelling older adults: results of a randomized, controlled trial. J Am Geriatr Soc. 2002;50(12):1921-8.

30. Gill TM, Baker DI, Gottschalk M, Peduzzi PN, Allore H, Van Ness PH. A prehabilitation program for the prevention of functional decline: effect on higher-level physical function. Arch Phys Med Rehabil. 2004;85(7):1043-9.

31. Pahor M, Blair SN, Espeland M, Fielding R, Gill TM, Guralnik JM, et al. Effects of a physical activity intervention on measures of physical performance: Results of the lifestyle interventions and independence for Elders Pilot (LIFE-P) study. J Gerontol A Biol Sci Med Sci. 2006;61(11):1157-65.

32. Olde Rikkert MG, Rigaud AS. Malnutrition research: high time to change the menu. Age Ageing. 2003;32(3):241-3.

33. Heuvelen van MJ, Kempen GI, Brouwer WH, de Greef MH. Physical fitness related to disability in older persons. Gerontology. 2000;46(6):333-41.

34. Milne AC, Potter J, Avenell A. Protein and energy supplementation in elderly people at risk for malnutrition. Cochrane Database Syst Rev CD003288. 2005.

35. Keysor JJ, Jette AM. Have we oversold the benefit of late-life exercise? J Gerontol A Biol Sci Med Sci. 2001;56(7):M412-23.

36. Keysor JJ. Does late-life physical activity or exercise prevent or minimize disablement? A critical review of the scientific evidence. Am J Prev Med. 2003;25(3 Suppl 2):129-36.

37. Bean JF, Vora A, Frontera WR. Benefits of exercise for community-dwelling older adults. Arch Phys Med Rehabil. 2004;85(7 Suppl 3):S31-42; quiz S3-4. 


\section{CHAPTER 6}

\section{Interventions to prevent disability in frail community-dwelling older people: an}

overview

This chapter was published as:

Daniels R, Metzelthin S, van Rossum E, de Witte L, van den Heuvel W. Interventions to prevent disability in frail community-dwelling older people: an overview. European Journal of Ageing 2010;7:37-55 


\begin{abstract}
This narrative review was conducted to provide an overview of the variety of interventions aimed at disability prevention in community-dwelling frail older persons and to summarise promising elements. The search strategy and selection process found 48 papers that met the inclusion criteria. The 49 interventions described in these 48 papers were categorised into 'comprehensive geriatric assessment', 'physical exercise', 'nutrition', 'technology' and 'other interventions'. There is a large diversity within and between the groups of interventions in terms of content, disciplines involved, duration, intensity and setting. For eighteen of the 49 interventions significant positive effects for disability were reported for the experimental group. Promising features of interventions seem to be: multidisciplinary and multifactorial, individualised assessment and intervention, case management, long-term follow-up, physical exercise component (for moderate physically frail older persons) and the use of technology. Future intervention studies could combine these elements and consider the addition of new elements.
\end{abstract}




\section{Introduction}

Frail older persons people are at much higher risk of disabilities, hospitalisation, institutionalisation, and death, compared with their age-matched non-frail counterparts. $^{1-3}$ In scenarios that predict future health service delivery in the Western world, the rapid increase in frail older persons is seen as one of the major challenges to health care. ${ }^{4-6}$ There has been an exponential rise in the use of the term 'frailty' in the literature. ${ }^{7}$ Markle-Reid and Brown ${ }^{5}$ reported substantial disagreement in the literature as to how frailty is defined and measured. The debate has focused on whether frailty should be defined purely in terms of biomedical factors or whether psychosocial factors should be included as well. ${ }^{8}$ From their literature reviews, Levers et al. ${ }^{9}$ as well as Aminzadeh et al. ${ }^{10}$ conclude that most definitions of frailty do include the idea of loss of age-related reserve capacity, though differences exist regarding other factors contributing to frailty. Despite a lack of consensus about the definition of frailty, a growing number of intervention studies for frail older persons is reported, implying that interventions can be targeted at frail older persons independent of specific diseases. Disability, defined as experienced difficulty in performing activities in any domain of life ${ }^{11}$, is generally considered as one of the major adverse outcomes of frailty. Prevention of disability in frail older persons is seen as a priority for research in geriatrics ${ }^{12}$ and can lead to the maintenance of quality of life and reduced health care costs. ${ }^{13}$ Several systematic reviews are available that focus on specific categories of interventions for frail older persons e.g. comprehensive geriatric assessment ${ }^{14}$, after-care ${ }^{15}$ or respite care. ${ }^{16}$ No overview is available, however, that provides an extensive overview of the content of the full range of existing programmes for community-living frail older persons that are aimed at the prevention of disability. The present study is a narrative review covering a wide range of programmes for community-dwelling frail elderly. The primary aim of the study is to provide an overview of the type of interventions studied in randomised or controlled clinical trials regardless of other aspects of their methodological quality. To develop future effective interventions aimed at disability prevention lessons can be learned from such studies. Therefore the secondary aim of the review is to summarise promising components for future interventions from studies that reported significant effects.

\section{Methods}

\section{Search strategy}

On 3 March 2008 the databases PubMed, the Cochrane Central Register of Controlled Trials (CENTRAL) and CINAHL were searched for randomised controlled clini- 
cal trials by use of the words 'frail*', ' vulnerable', 'at risk", ' high risk", 'low functioning', and the MESH terms 'chronic disease' and 'disabled persons' in combination with the MESH term 'aged'. Search terms for outcomes focused on disability measures and included terms like 'disabil*', 'functional decline', 'functional capabilit*', 'functional performance', 'independen*' and MESH terms 'activities of daily living', 'quality of life' and 'well being'. To restrict the search to interventions that targeted community-dwelling older persons, terms like 'home*', 'in-home*', 'communit*', ' "independent living' and MESH term 'primary care' were added. Additionally studies were identified by a manual search of reference lists from relevant papers. The search was restricted to articles in English, Dutch and German. There was no restriction on type of intervention or year of publication.

\section{Selection criteria}

Inclusion criteria were set for study population, outcome measure and design. Randomised and controlled clinical trials specifically aimed at community-dwelling frail older persons were included. No restrictions were set concerning the definition of frailty. As frailty points to an increased risk of adverse outcomes, only studies that specified the criteria used to operationalise the increased risk were included. Studies that used physical markers to include participants were included, as well as studies that used a combination of factors (multifactorial perspective on frailty) as inclusion criteria. Exclusion criteria for the population concerned the selection of participants solely based on age, age and fall incidents, and age and having one chronic disease.

Disability was used as the outcome measure (regardless of whether it was used as a primary or secondary outcome) and defined as difficulty experienced in performing activities. ${ }^{11}$ Avlund ${ }^{17}$ found that most current studies of disability among older persons focus on the ability to carry out activities of daily living. In this review, studies reporting measurements of Activities of Daily Living (ADL) or Instrumental Activities of Daily Living (IADL) were included.

\section{Data extraction and analysis}

A first selection of relevant studies was made by RD on title-level with a conservative approach, meaning that in case of doubt an article would always be screened on abstract-level. The second (abstract-level) and third selection phase (full-text level) were independently undertaken by two reviewers (RD and SM) scoring 'relevant', 'doubt' or 'irrelevant' on forms. In case of inconsistencies, the reviewers discussed their scores. Consensus on 'irrelevant' led to the exclusion of an article. On several occasions the reviewers asked for the involvement of a third party (EvR) in order to reach consensus. 
The same two reviewers performed the data extraction with respect to the aims, target population, design, care disciplines involved, and content of the interventions. Furthermore, follow-up and reported effectiveness on disability was retrieved from the articles. Assessment of the methodological quality of studies was not performed, as the primary aim was to provide an overview of the type of interventions reported for community-dwelling frail older persons. The research team (RD, SM, EvR, LdW, WvdH) discussed ways of categorising the studies based on descriptions common in geriatric literature. As this review intends to provide an overview of the content of interventions, it was decided to categorise the interventions according to their intervention characteristics. Interventions were classified into 'comprehensive geriatric assessment (CGA)', 'physical exercise', 'nutrition', 'assistive technology' and 'other interventions'. Studies that reported significant effects in favour of the experimental group on ADL or IADL measures were further explored (by RD and SM) to identify intervention elements that might explain successful outcomes.

\section{Results}

Four thousand, six hundred and forty-five articles were identified in the literature search. After screening of the titles, 170 studies were considered relevant for further screening on abstract-level. Of these, another 63 studies were excluded, because they did not meet the inclusion criteria (Figure 1). In the next phase, the screening of 107 full-text articles resulted in the exclusion of 59 studies. Of these, 21 were excluded as they did not meet the criteria for population characteristics; fourteen did not meet the criteria for the outcome measure (disability) and fourteen failed owing to study design. Forty-eight studies, describing 49 interventions, were included. Among these 26 interventions were categorised as 'comprehensive geriatric assessment (CGA)' twelve as 'physical exercise', three as 'nutrition', two as 'assistive technology' and six were classified as 'other interventions'. All studies were published between 1986 and 2008. There is a large variation in the criteria that studies used to include frail older persons (see Table 1). Physical frailty markers are more common as inclusion criteria in physical exercise programs, while more complex interventions (CGA) generally use a combination of factors, taking a multifactorial perspective on frailty. All 48 studies met de inclusion criterion to measure disability by using measurements on ADL or IADL. However, disability was not the primary outcome measure for all studies. Eleven studies did perform a long term follow-up measurement ( $\geq 6$ months after the end of the intervention). For nine studies information on follow-up was lacking. 


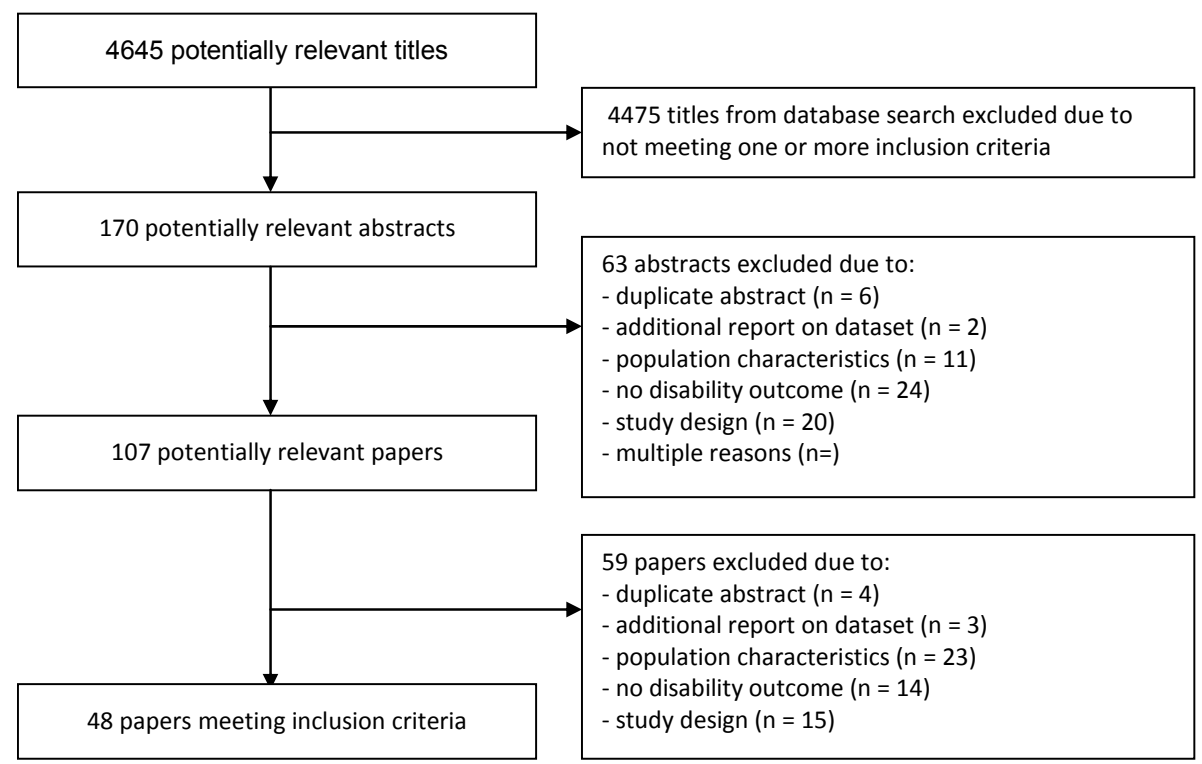

Figure 1 Progress of Search for Relevant Trials

\section{Intervention characteristics (see also Table 1)}

\section{Comprehensive Geriatric Assessment ( $N=26$ )}

Comprehensive geriatric assessment (CGA) has been defined as 'a multidimensional, often interdisciplinary, diagnostic process intended to determine a frail older persons person's medical, psychosocial, and functional capabilities and problems, with the objective of developing an overall plan for treatment and long-term followup $^{\prime}{ }^{18}$ For this review, the included CGA studies were further divided into transmural care and community-based care. In the latter, a distinction was made between studies in which assessment was followed by referrals or recommendations and studies where assessment was directly followed by treatment and care. 


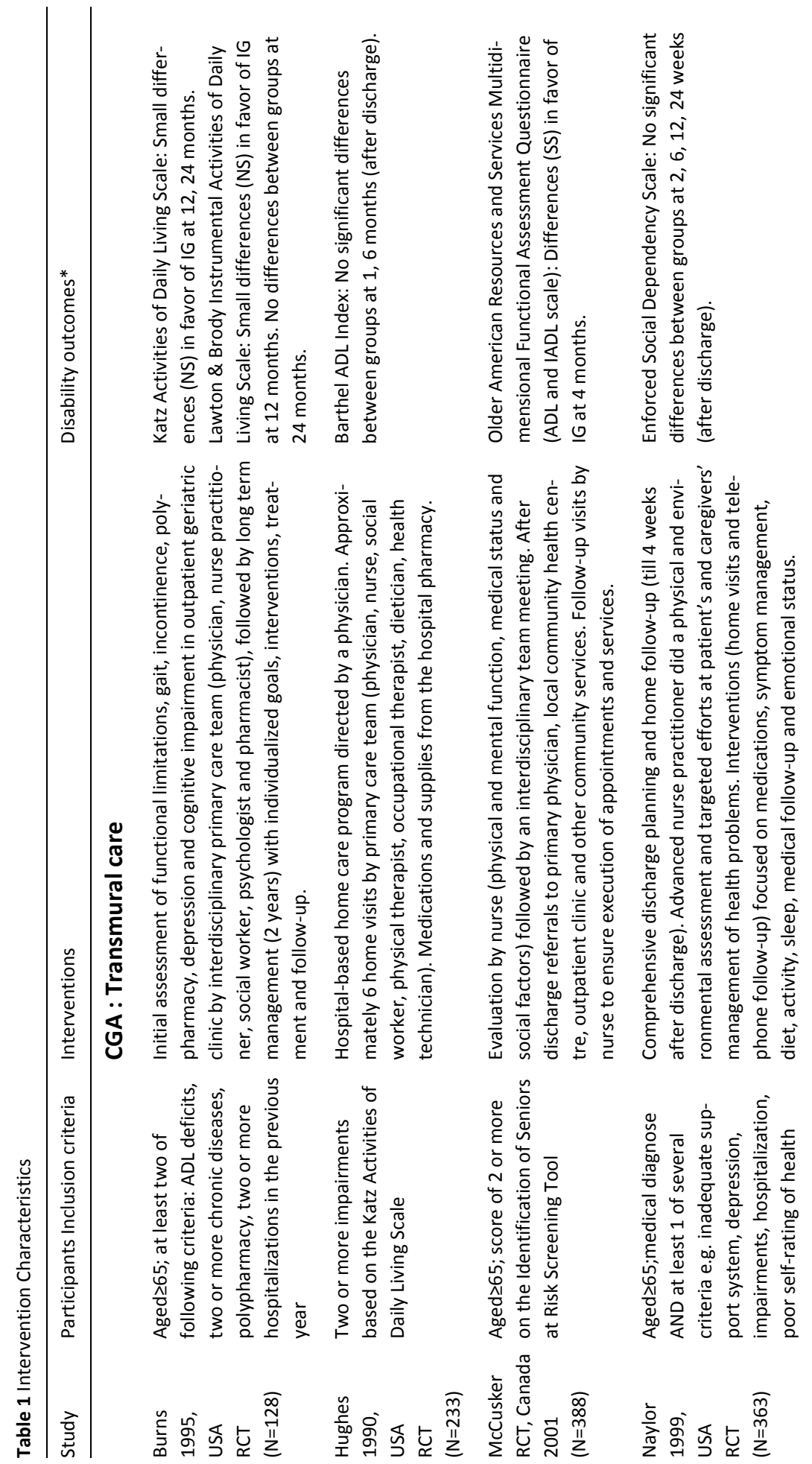




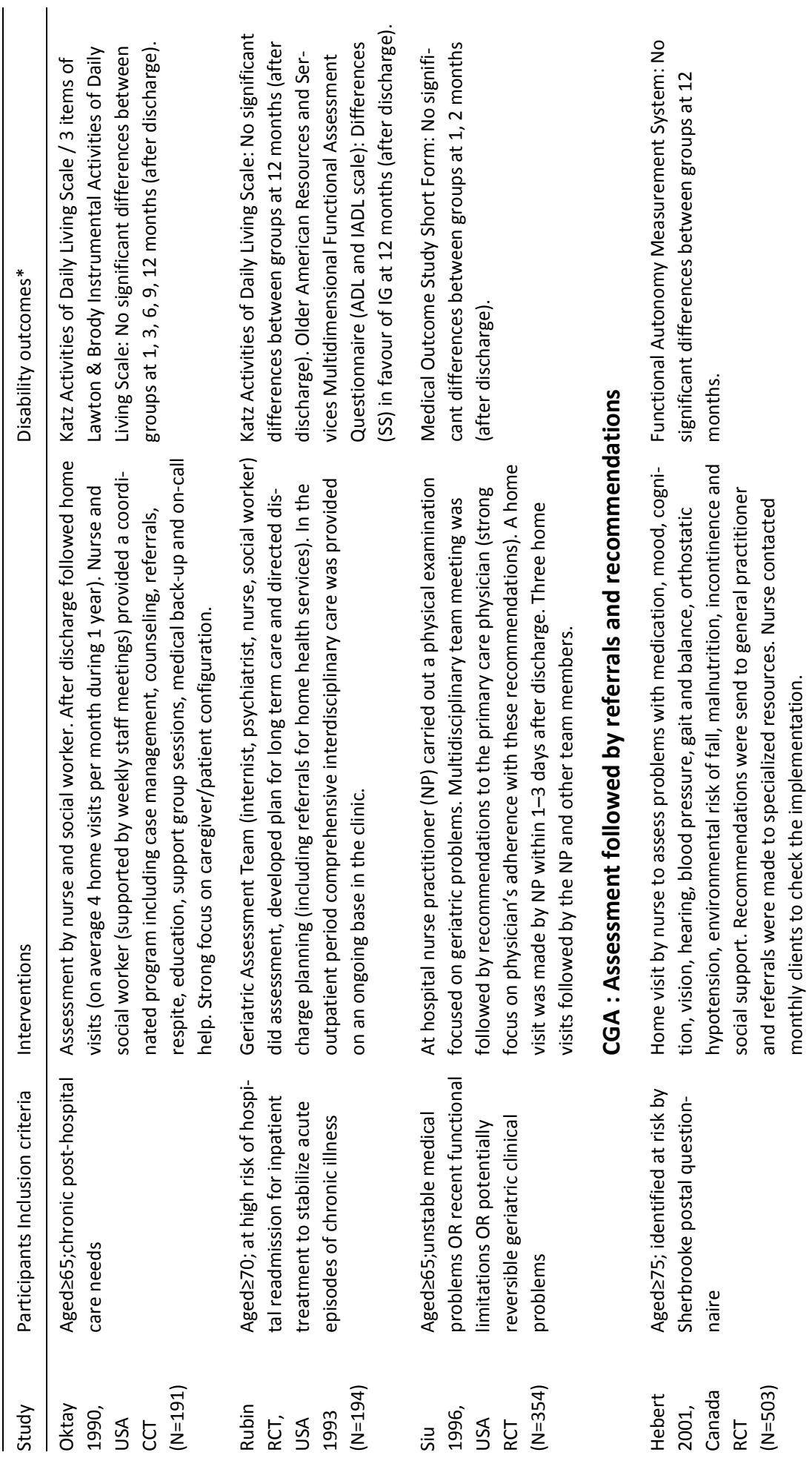




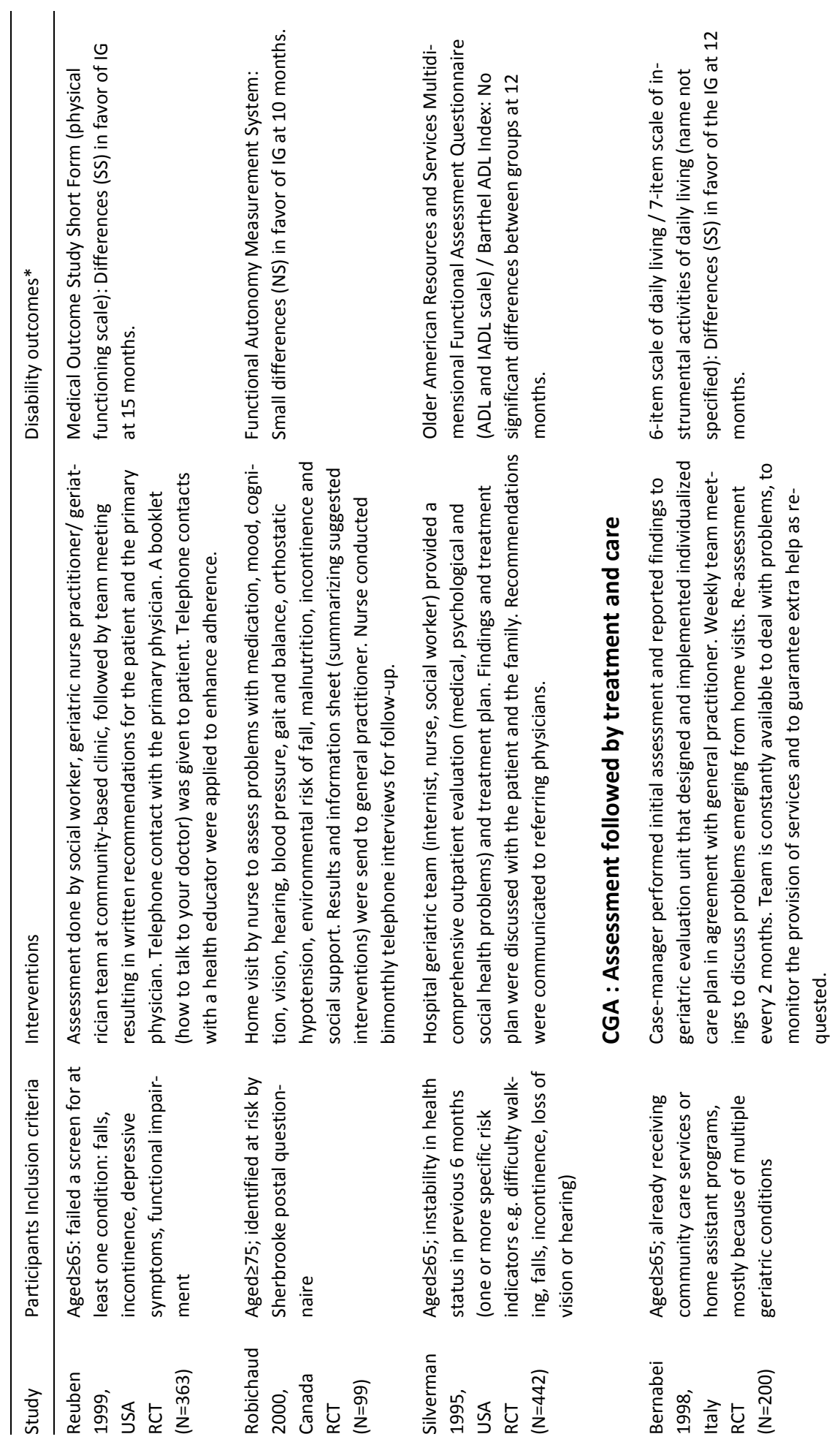




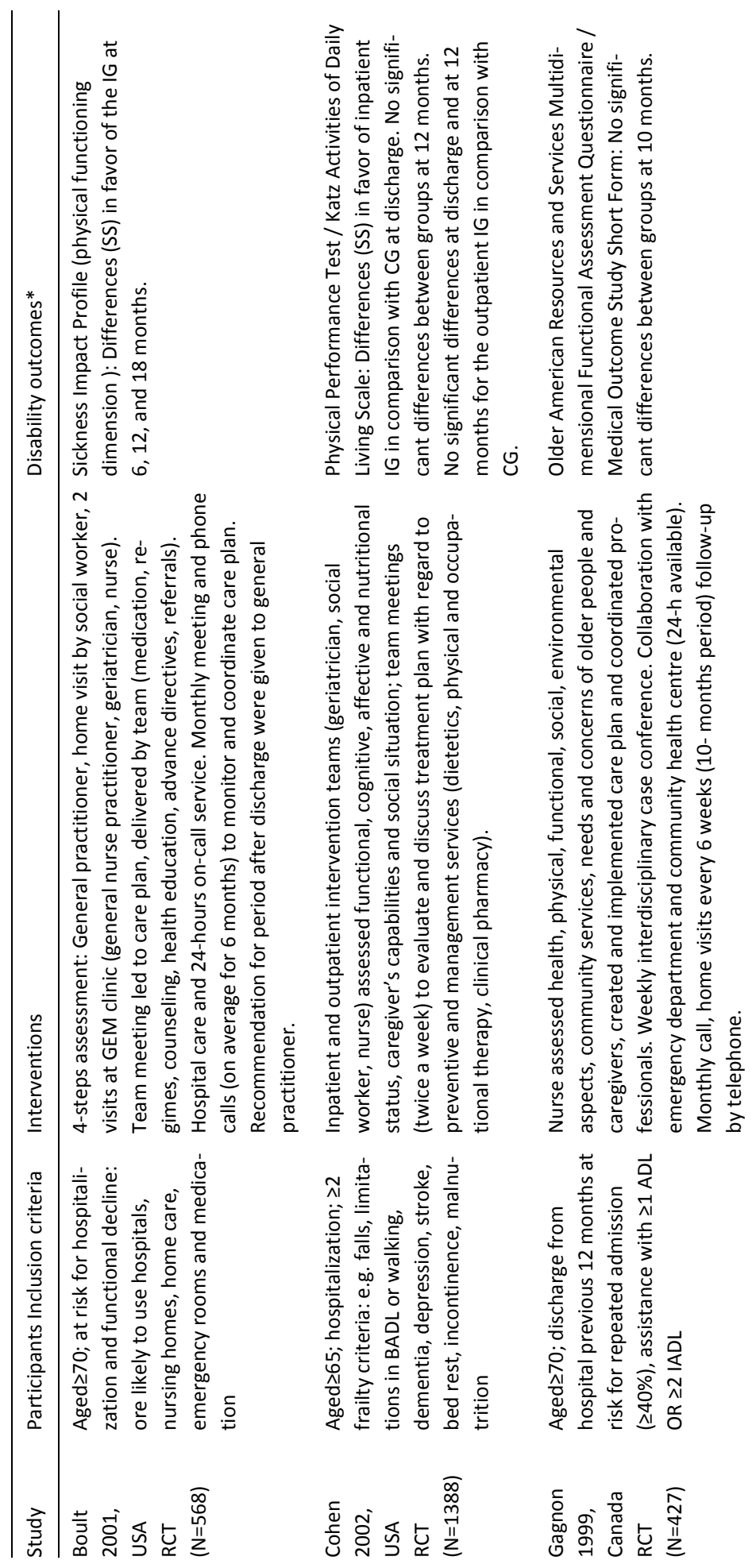




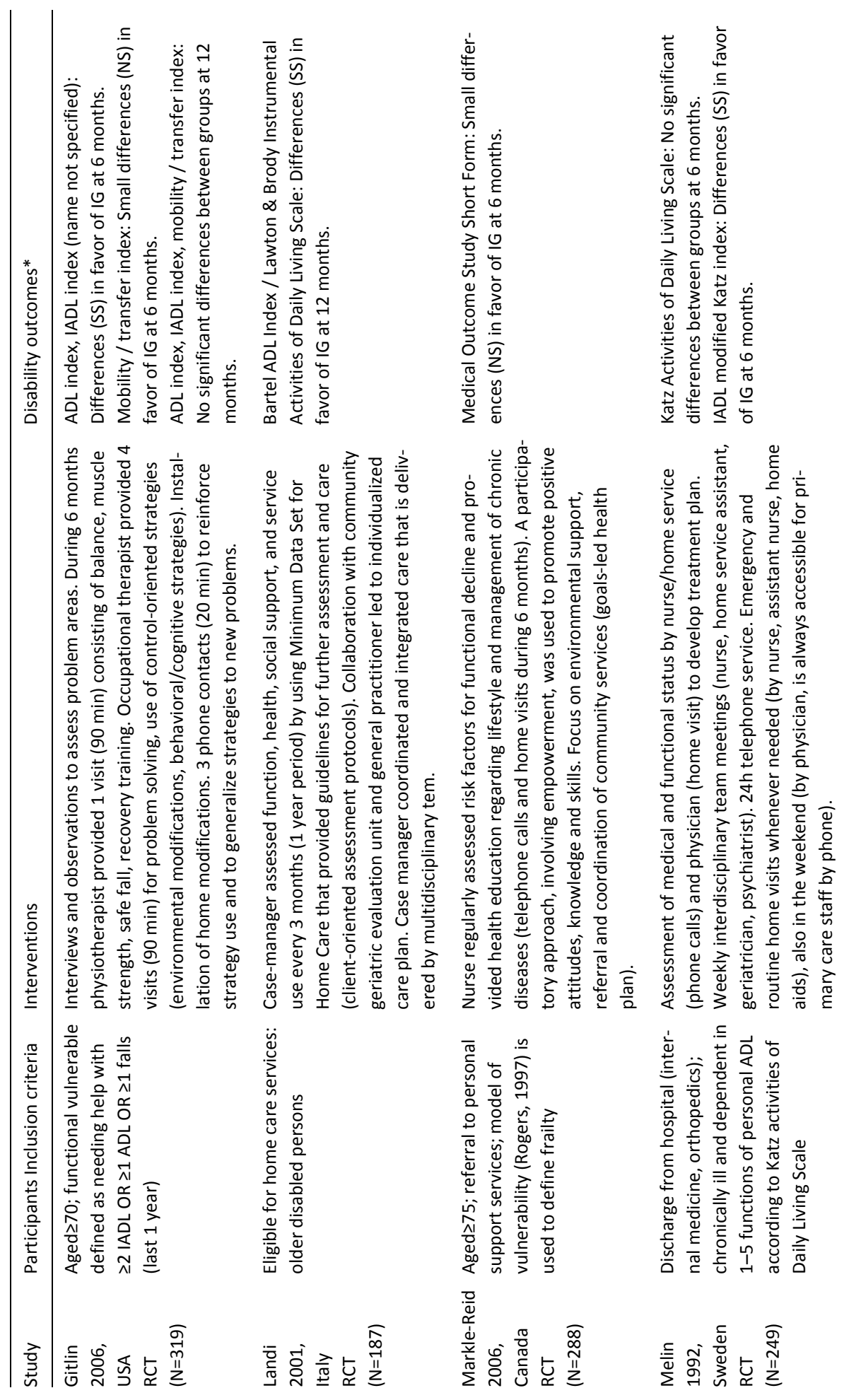




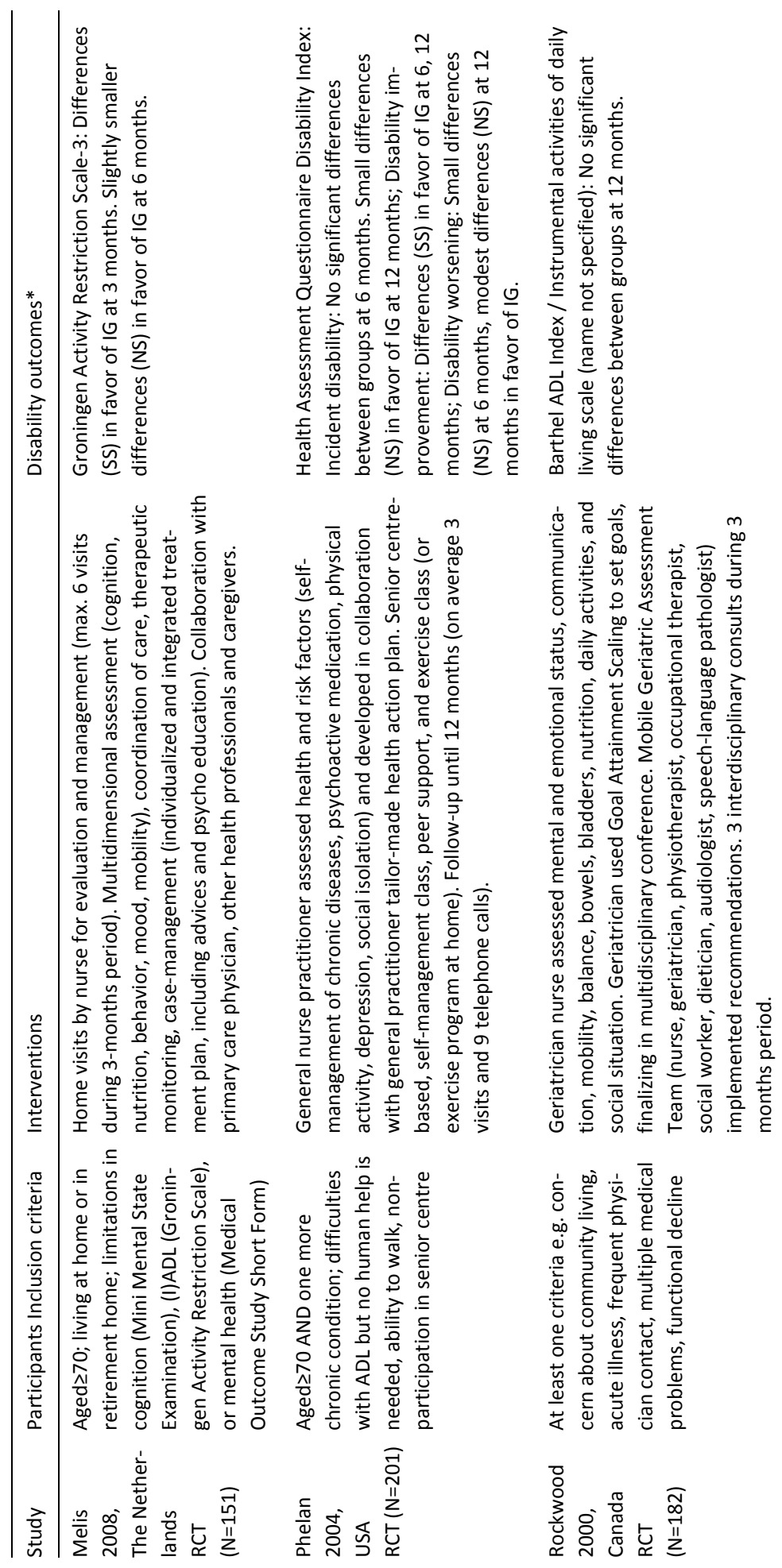




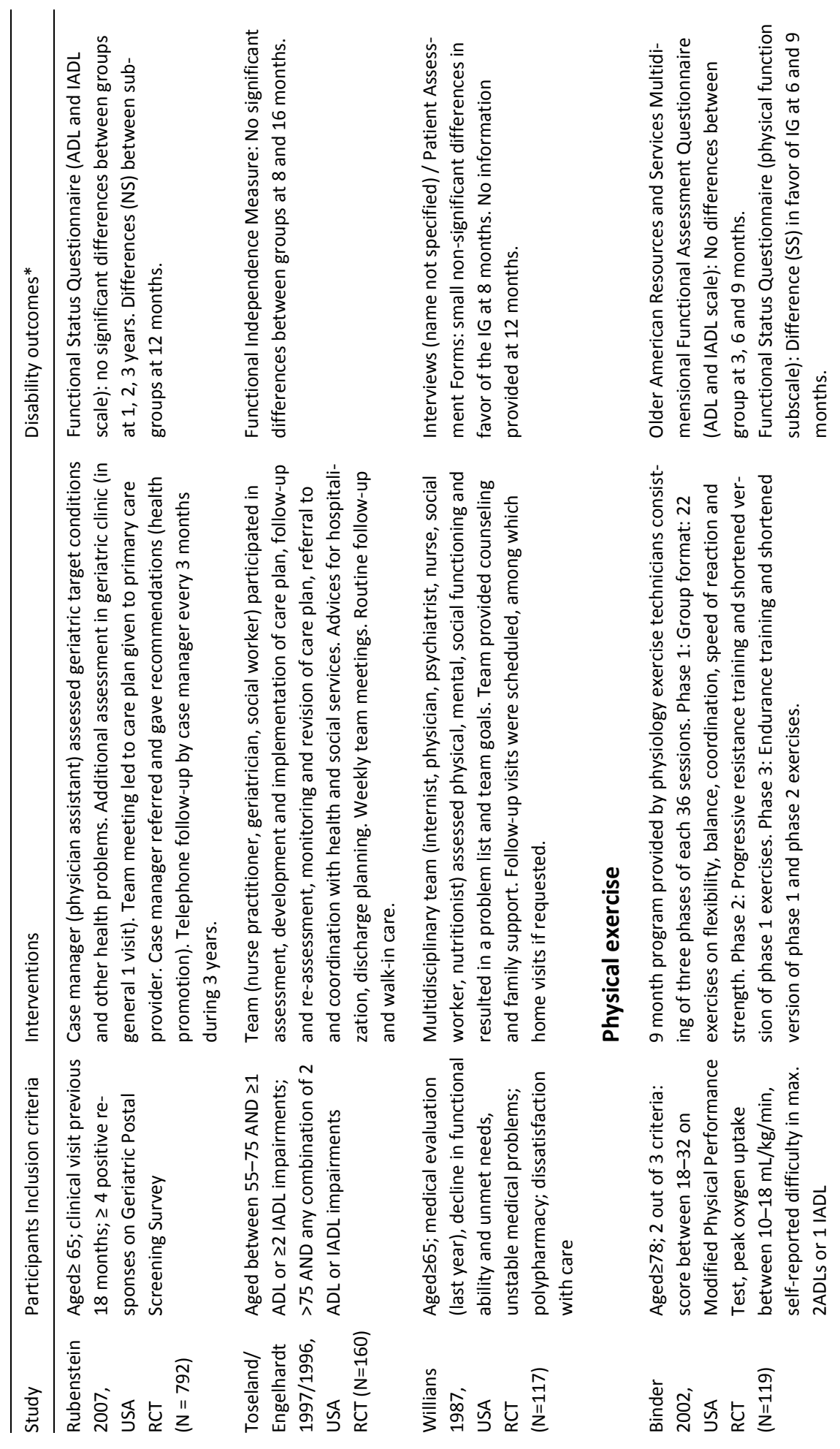




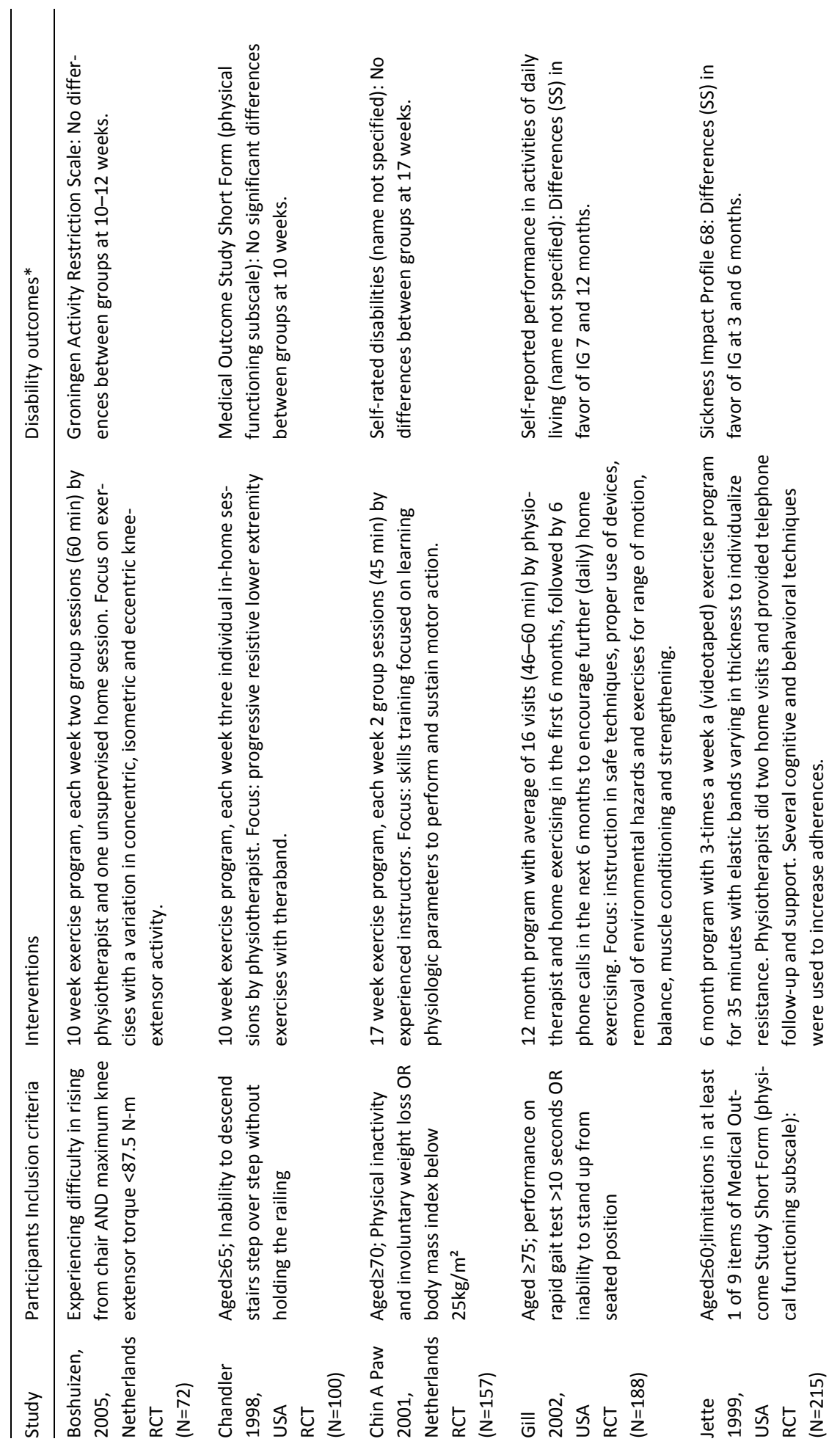




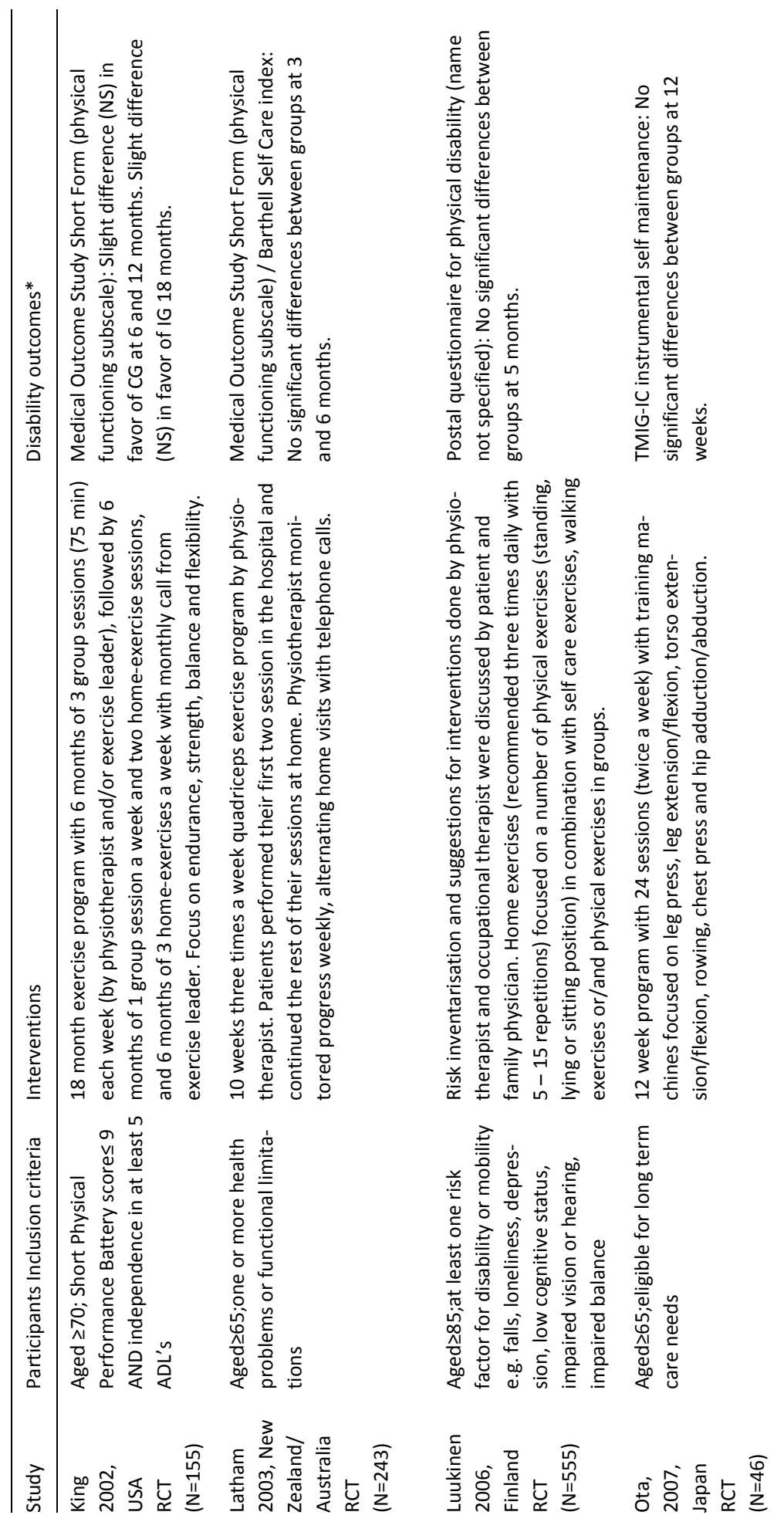




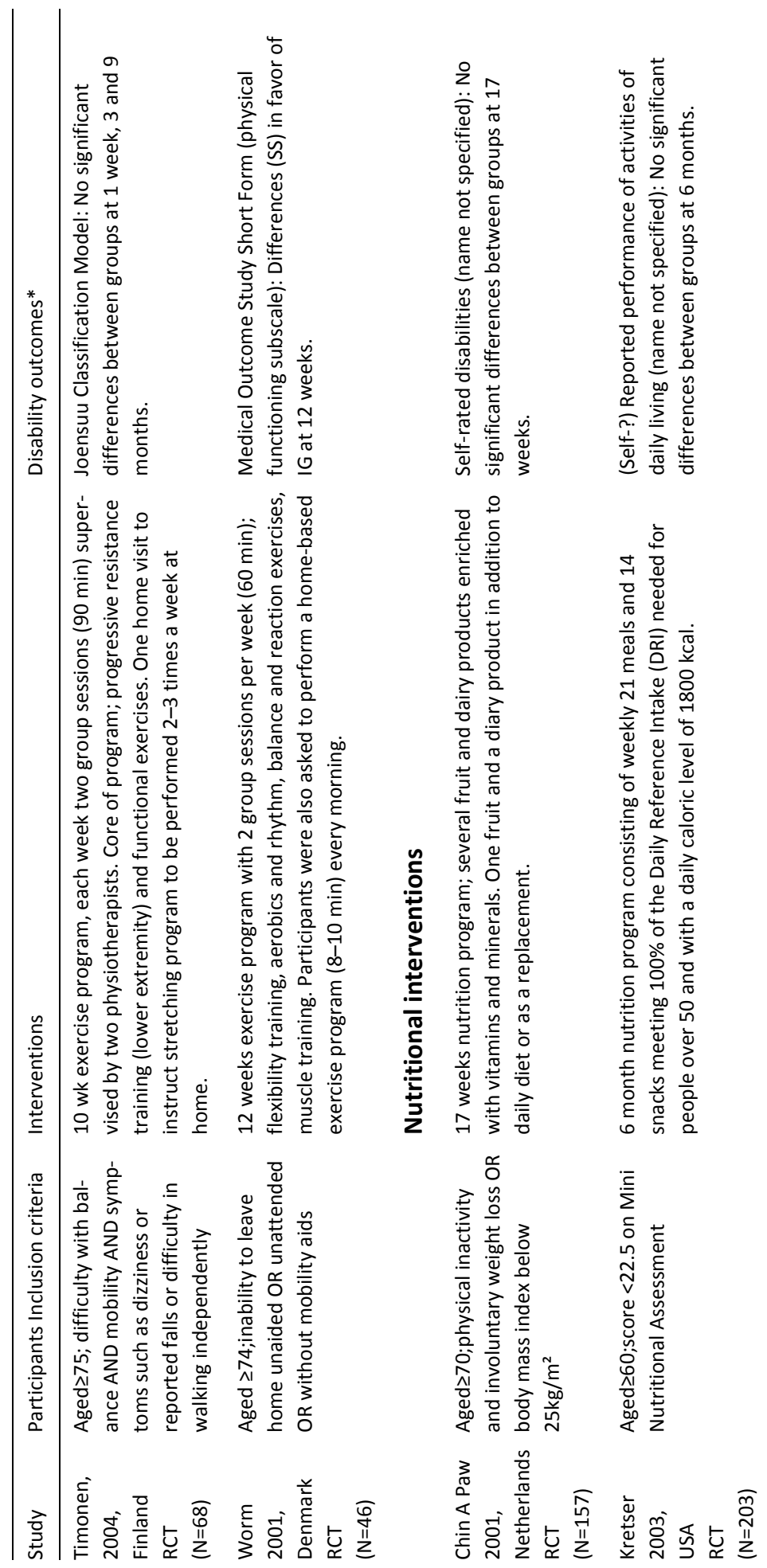




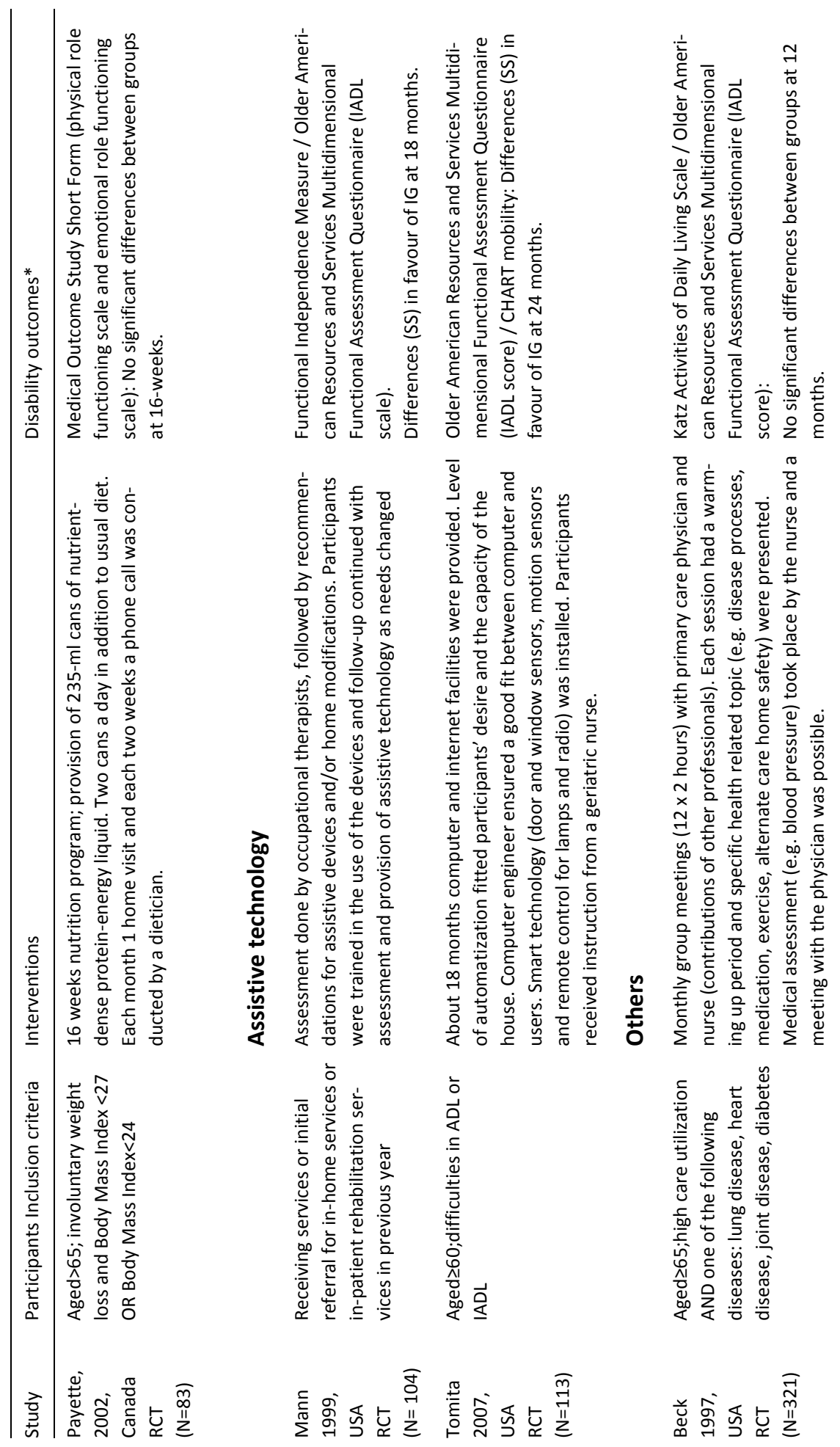




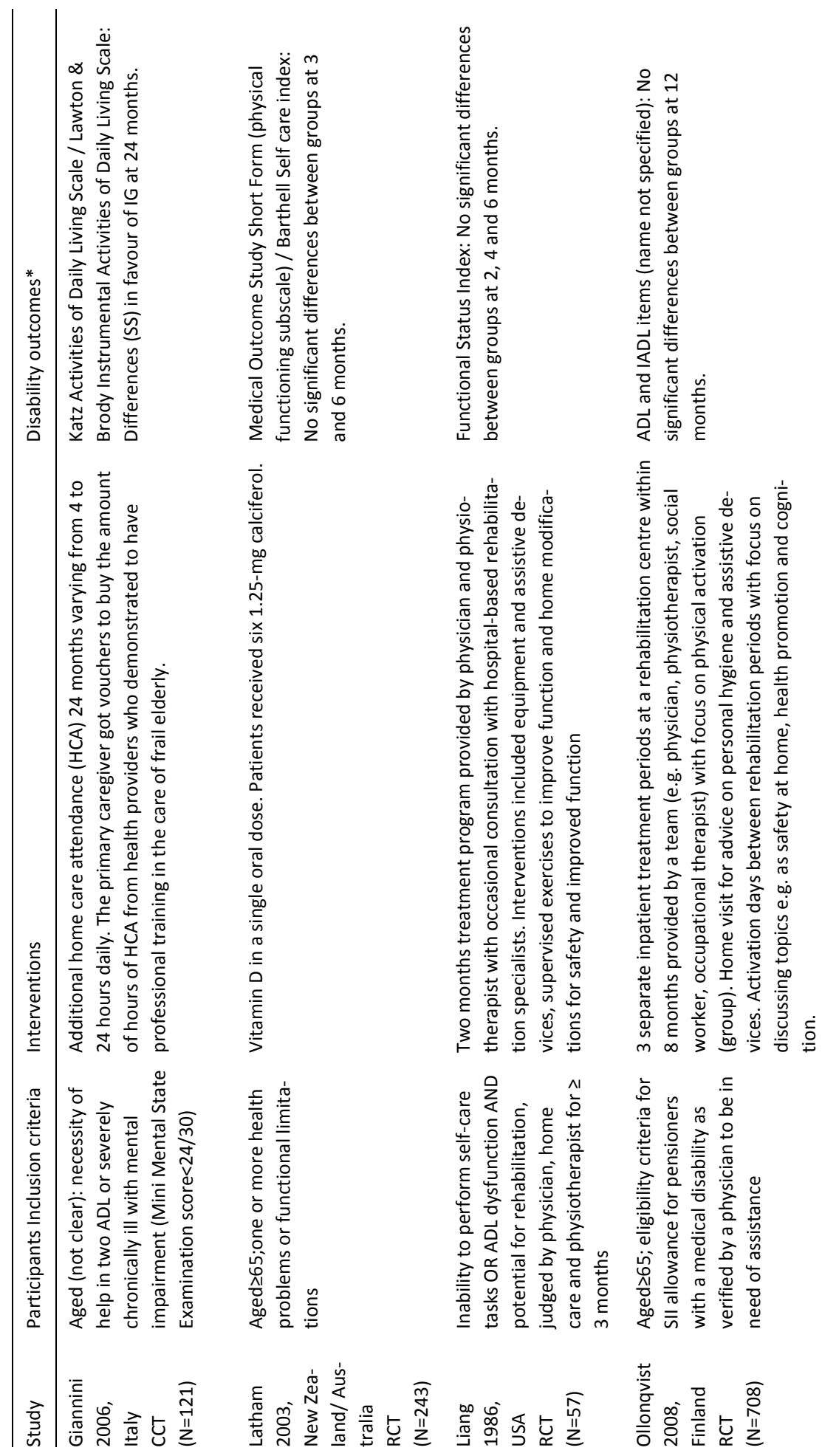




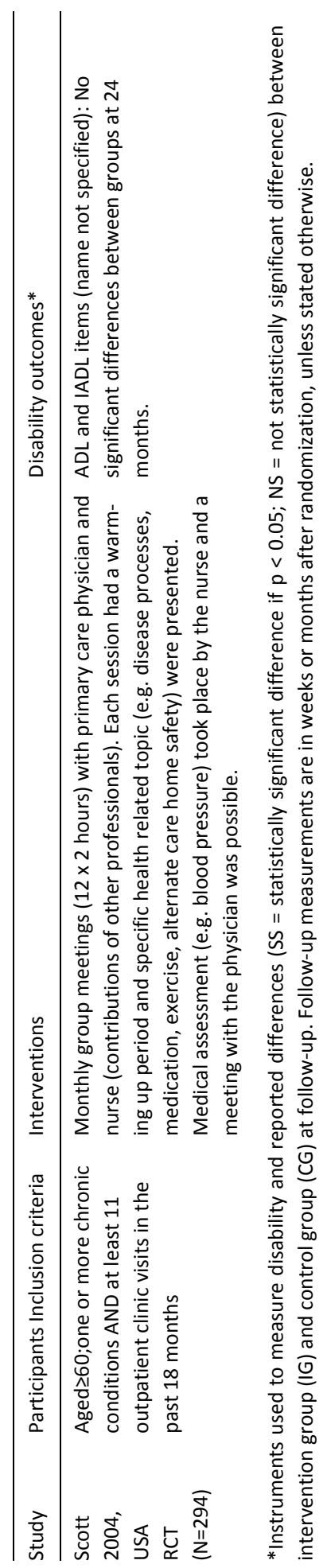


Transmural Care $(\mathrm{N}=7)$

In this review, transmural care points to interventions in which clients were identified and assessed during admission to the hospital setting. After discharge, client referrals were made and interventions were delivered in the community. In the seven studies, assessment was performed by a nurse practitioner ${ }^{19-21}$, a physician ${ }^{22}$ or an interdisciplinary team ${ }^{23-26}$ focusing on a variety of physical, mental, medical and social factors. Usually, the assessment was followed by a team meeting leading to an individualised treatment plan and a variety of actions e.g. referrals ${ }^{19}$ and recommendations to the general practitioner ${ }^{21}$, home visits by a nurse practitioner ${ }^{20}$ or several disciplines ${ }^{21,22,25}$ and long-term outpatient comprehensive care by a geriatric clinic. $^{23,24,26}$ Nursing interventions targeted, for example, medications, symptom management, diet, activity, sleep, medical follow-up and emotional status. ${ }^{20}$ The interdisciplinary team in the studies by Burns and colleagues ${ }^{23,24}$ focused on functional limitations, gait impairment, incontinence, polypharmacy, depression, cognitive impairments and caregiver needs.

Out of the seven transmural care interventions, one study reports significant effects in favour of the experimental group. ${ }^{19}$ Pointing out effective elements is difficult. In the study by McCusker and colleagues ${ }^{19}$, a two-step screening approach including the ISAR screening tool was used. This screening tool, specifically designed and evaluated for identifying seniors at high risk, might have increased the efficacy of the selection of participants.

In the study by McCusker and colleagues ${ }^{19}$, treatment was individualised and clients were seen by a variety of disciplines. Referrals were made to the physician, local community, health centre, geriatric outpatient clinic and other community services. Comparable features, however, are present in the other CGA 'transmural care' studies that did not report effectiveness on disability.

\section{Community-based care: Assessment followed by referrals and recommendations $(N=4)$}

Eligible participants for these four studies were identified by a self-administered postal questionnaire ${ }^{27,28}$, telephone interview ${ }^{29}$ or an interviewer-assisted selfadministered screening. ${ }^{30}$ Extensive assessment was performed by a nurse during home visits ${ }^{27,28}$ or by a team in community-based clinics ${ }^{29,30}$. Medication, mood, cognition, vision, hearing, blood pressure, gait and balance, orthostatic hypotension, environmental risk of fall, malnutrition, incontinence and social support were part of the assessment in the studies by Hebert and colleagues ${ }^{27}$ and Robichaud and colleagues. $^{28}$ In all studies, the assessment was followed by recommendations to the general practitioner. In the study by Hebert and colleagues ${ }^{27}$ and Robichaud and colleagues $^{28}$, recommendations to other health professionals were also made and bi-monthly or monthly phone follow-up interviews took place. ${ }^{27,28}$ In the study by 
Silverman and colleagues ${ }^{29}$, the treatment plan was discussed with the patient and family. Preparation of the patient and caregiver for the meeting with the general practitioner was part of the intervention in the study by Reuben and colleagues. ${ }^{30}$

Out of these four community-based CGA studies, only the study by Reuben and colleagues $^{30}$ found significant differences in disability in favour of the experimental group. The near-significant effect that Robichaud and colleagues ${ }^{28}$ found did not reach significance in the study by Hebert and colleagues ${ }^{27}$ in the evaluation of the same intervention among a larger group of participants. As all other three interventions have similar features to those of Reuben and colleagues ${ }^{30}$, elements that produce effectiveness in this type of CGA cannot be identified.

\section{Community-based care: Assessment followed by treatment $(N=15)$}

Two out of fifteen studies are about the same intervention. ${ }^{31,32}$ All interventions were delivered by an interdisciplinary team consisting of at least two disciplines. Members of the interdisciplinary team were, for example, general practitioners, geriatricians, nurses, nurse practitioners, social workers, allied health professionals or medical specialists. The core of the intervention was mainly delivered by primary care professionals. Professionals working in institutions, for example, a geriatric clinic, were, however, often involved in the assessment and the development of the treatment plan, including referrals and recommendations. In addition, they could be consulted about the delivery of supplementary treatment if needed. In many interventions one person from the interdisciplinary team, often a nurse (practitioner), had the role of a case-manager. ${ }^{33-40}$ The case-manager was responsible for planning, coordination, monitoring, and evaluation of assessment and treatment. The assessment in the fifteen studies focused on medical conditions and general status of the participants (functional, psychosocial, cognitive, affective and nutritional), personal resources and preferences, caregiver's capabilities and other environmental factors. The focus of the interventions varied strongly across studies. Issues covered were falls, balance, urinary incontinence, functional impairment, depression, cognitive deficits, nutrition, mobility, medication, social support, service use, communication, environmental aspects and financial needs.

Nearly all interventions were delivered in an individual format, except for Phelan and colleagues ${ }^{39}$, who combined individual sessions with group sessions (exercise and self-management classes). Home visits ${ }^{33,35,38,41,42}$, telephone calls ${ }^{40}$ or a combination of both ${ }^{36,37,39,43}$ are repeatedly used to address frail older persons. In three studies assessment and treatment were done in a (geriatric) clinic. ${ }^{31,32,44}$ Two studies combined clinic visits, home visits and telephone contacts. ${ }^{40,45}$

Out of fifteen CGA studies, nine report significant differences in disability in favour of the experimental group. ${ }^{33,35-39,43-45}$ There are some features that promising interventions have in common. Out of the nine studies, seven studies report the use of 
an individualised treatment plan that is based on a multidimensional assessment. ${ }^{33,35,37,38,44-46}$ A case-manager had a key role during the intervention process in six out of nine effective studies. ${ }^{33,35-39}$ Regular team meetings were applied in four studies. $^{33,37,38,44}$ In seven out of the nine studies, ongoing assessment, evaluation and monitoring are described as a feature of the intervention. ${ }^{33,35,36,38,45-47}$ Therefore a combination of home visits and telephone contacts is often used. ${ }^{36,37,39,40,43}$ Out of nine studies, four interventions also intervene in factors in the social and physical environment of frail older persons. ${ }^{36,38,45,46}$ Health education is applied in four interventions. ${ }^{36,38,40,43,45}$ A complication in drawing conclusions about elements that contributed to effectiveness is the presence of identical features in the six clinical trials that did not show any differences for disability. ${ }^{31,32,34,40-42}$ There is, however, some indication that case management, individualised treatment, multidimensional assessment and ongoing evaluation and monitoring are relevant features in this type of CGA interventions. A combination of home visits and telephone contacts and regular team meetings seems to be promising. Health education may also be an important component for future interventions.

\section{Physical Exercise programmes $(N=12)$}

Twelve studies describing physical exercise interventions were found. Physical exercise interventions for community-dwelling frail older persons show a large variation in content, duration, intensity, balance between supervised and non-supervised sessions and level of individualisation. Five interventions were single-component, focusing mainly on lower extremity strength. ${ }^{48-52}$ The other seven were multicomponent, addressing a variety of physical parameters such as endurance, flexibility, balance and strength. ${ }^{53-59}$ All interventions were offered by professionals in physical exercise, mostly physical therapists. In most studies, participants performed at least three exercise sessions a week. ${ }^{48-51,54-59}$ Strength training was usually comprised of a number of exercises for improvement of lower body strength using weights ${ }^{60}$, elastic bands ${ }^{48-50}$ or training machines ${ }^{52}$. Among the multicomponent exercise programmes, three addressed several parameters in all exercise sessions. ${ }^{56,57,59}$ These also belong to the shortest multicomponent exercise programmes (10-12 weeks). The longest multi-component programmes lasted nine months $^{58}$, twelve months ${ }^{54}$ and eighteen months. ${ }^{55}$ In two studies ${ }^{55,58}$, different phases (respectively three or six months) were distinguished with a focus on specific physical parameters in each phase. The content of the programme of Gill and colleagues $^{54}$ was more individualised, as the outcomes of an extensive assessment directed the programme. Among the physical exercise programmes, there is a large difference between the number of supervised and non-supervised sessions. For example, Binder and colleagues ${ }^{58}$ report a total of 108 supervised sessions, while Jette and colleagues ${ }^{50}$ report only two home visits by a physical therapist. The par- 
ticipants exercised non-supervised, supported by techniques to enhance adherence. $^{50}$

Out of twelve physical exercise programmes, four report significant positive effects for disability. Of the single-component physical exercise programmes $(n=5)$, one reports positive effects. Jette and colleagues ${ }^{50}$ found evidence for the effect of resistance training using elastic bands. For three (out of seven) multi-component programmes, significant positive effects are reported on the disability outcome. ${ }^{54,56,58}$ In these three studies, participants were included according to physical frailty indicators and programmes were relatively intensive with at least three exercise sessions per week. Binder and colleagues ${ }^{58}$ included only moderate physically frail older persons; Gill and colleagues ${ }^{54}$ found that only moderate physically frail older persons benefited from their intervention.

\section{Nutrition $(N=3)$}

Studies that investigated the effect of single nutritional interventions focused on macronutrient status (nutrient-dense protein energy liquid: ${ }^{61}$ ), micronutrient status (fruits and dairy products enriched with vitamins and minerals: ${ }^{53}$ ) or both (meals and snacks providing $100 \%$ of macro- and micronutrient requirements ${ }^{62}$ ). In the study by Kretser and colleagues ${ }^{62}$ participants received 21 meals and fourteen snacks every week for six months, accounting for most of the daily nutritional intake. In Chin A Paw and colleagues' study ${ }^{63}$, participants were asked to eat the products in addition to their daily diet or as a replacement (for seventeen weeks). In Payette and colleagues ${ }^{61}$, the liquid product was an addition to the usual daily dietary intake for sixteen weeks. Additional support included monthly home visits by dieticians and a phone call every two weeks with nutritional counselling and encouragement ${ }^{61}$ or daily additional phone calls from older adult volunteers to provide a measure of safety and socialisation. ${ }^{62}$ Despite an observed effect on total energy intake $^{61}$ and weight gain ${ }^{61,64}$, none of the three nutritional intervention studies ${ }^{53,61,64}$ report evidence for the effect of nutritional interventions on disability.

\section{Technological interventions ( $N=2)$}

Environmental adaptations are often part of the multifactorial and multidisciplinary programmes described under CGA. Two reports were found on the effect of single assistive devices and home modifications ${ }^{65}$ and smart technology on disability. ${ }^{66}$ In the study by Mann and colleagues ${ }^{65}$, an occupational therapist performed a comprehensive functional assessment of the person and the home followed by recommendations for assistive devices and/or home modifications. Participants were trained in the use of the devices and follow-up continued with assessment and provision of assistive technology as needs changed. Over about eighteen months computer and internet facilities were provided to the participants in the study by Tomita 
and colleagues. ${ }^{67}$ A computer engineer adapted the computer to ensure was a good fit with its users. Furthermore, smart technology, like door and window sensors, motion sensors and remote control for lamps and radio, was installed. The level of automatisation was determined by the participants' desire and the capacity of the house. Participants received instruction from a geriatric nurse who was a specialist in computer instruction.

Both studies ${ }^{65,66}$ report significant differences on disability in favour of the experimental group. In both interventions there is an emphasis on the adaptation of technology towards the needs of the clients and on intensive instruction in the use of devices.

\section{Other interventions $(N=6)$}

In this group, six interventions are described. Latham and colleagues ${ }^{51}$ studied the effect of Vitamin D. Participants in the experimental group received $1.25 \mathrm{mg}$ calciferol. Giannini and colleagues ${ }^{68}$ focused on the effect of home care attendance (between four and 24 hours) by professionals with training in the care of frail older persons. In two studies ${ }^{69,70}$ the intervention consisted of monthly group meetings (over twelve months) with a physician and a nurse. The meetings focused on health promotion and control. One intervention ${ }^{71}$ contains three inpatient rehab periods with individual and group sessions plus home activation days and home visits for personal hygiene and assistive devices ${ }^{72}$ used a stepped-up treatment (on top of nursing and social services) provided by a physician and physical therapist focusing on assistive devices and home modification and supervised exercises.

Out of the six interventions, Giannini and colleagues ${ }^{68}$ report significant positive effects on disability in favour of the experimental group. They describe how the home care attendance, provided by a nurse trained in the care of frail older persons, was delivered according to a programme established by a Geriatric Evaluation Unit. Specific features of this programme are lacking.

\section{Discussion}

This review offers a comprehensive overview of the content of interventions targeted at disability prevention in community-dwelling frail older persons. In total, 48 clinical trials evaluating 49 interventions aimed at disability prevention were identified. The majority of trials in 46 RCTs and two CCTs were conducted in the field of comprehensive geriatric assessment (CGA) ( $n=26)$. Studies of physical exercise programmes are the second largest group of interventions $(n=12)$. There is a small number of studies that specifically focus on the effect of technology ( $n=2)$ or nutrition ( $n=3$ ) on disability in community-living frail older persons. Both environmental adaptations and nutrition, however, are frequently mentioned as part of the CGA 
studies. The results show a large diversity within and between the groups of interventions in terms of content, disciplines involved, duration, intensity and setting.

The reported effectiveness of interventions is not consistent. Only eighteen of the 49 interventions reported significant positive effects for disability for the experimental group. As most studies did not include long term follow measurement the preventive potential of interventions remains unclear for the time period after the completion of the intervention.

The most promising findings were found for technology (but only two studies), CGA 'assessment followed by treatment' (nine out of fifteen studies) and, to a lesser extent, physical exercise programmes (four out of twelve).

Technology, adapted to the needs of the frail older persons and well-taught, may be very effective in preventing disability in community-dwelling frail older persons, though more research is needed in this area.

Most trials were conducted in CGA, but the reported effectiveness of CGA for community-dwelling frail older persons is inconsistent. Comparable findings are reported in previous reviews on $\mathrm{CGA}^{14,73,74}$. Individualised, multifactorial and multidisciplinary assessments and interventions, case management and ongoing monitoring (long-term management) seem to be essential elements for effective CGA.

The mixed results of physical exercise programmes hamper the identification of effective elements. There is, however, some indication that multi-component high intensive physical exercise programmes may be promising, especially for moderate physically frail community-dwelling older persons.

This overview has some limitations. The use of the term frailty in the literature is relatively new. Although reference lists were checked, a limitation of this overview is that older studies might not have been identified as we searched with terms for frailty and its synonyms. Another risk in terms of publication bias is the selection of 4602 studies on title-level that may have resulted in the exclusion of relevant articles. Only randomised and controlled clinical trials were selected as we considered these as a (minimal) standard for quality. Some caution in interpretation of results is warranted as the methodological quality of the studies was not assessed. Furthermore we analyzed the results in a qualitative way. As a consequence effect sizes of interventions were not calculated or taken into account. And last, owing to small sample sizes, trials may have been underpowered to detect differences in the selfreported measures for disability.

Recent literature on disability development suggest that disability is multifactorial in nature. Stuck et al. ${ }^{75}$ reported that risk factors for developing disability in community-dwelling elderly are cognitive impairment, depression, co morbidity, increased 
and decreased body mass index, lower extremity functional limitation, low frequency of social contacts and low level of physical activity. Femia et al. ${ }^{76}$ suggest that although disease conditions and physical impairments are as risk factors strongly related to an individual's ability to carry out activities of daily living, other factors like the beliefs about one's health (e.g., subjective health), motivation and self-efficacy are potentially as important as the ability to perform them. Therefore, it seems that a combination of risk factors and other factors plays a role in the development of disability implying that disability prevention in frail elderly is complex.

In view of this complexity the Dutch National Health Council recently pointed out the need for research on "function-oriented prevention". The Health Council states that knowledge of the effectiveness of preventive interventions on disability is fragmented, heterogenic and still lacking in a variety of areas. ${ }^{77}$

How might the findings of this overview influence future interventions for community-dwelling frail older persons? In the light of this overview, future interventions may be directed towards tailor-made, multidisciplinary and multifactorial interventions, with individualised assessment and interventions conducted by a (primary) care team, involving case management and long-term follow-up. These tailor-made programmes may include a physical exercise component for moderate physically frail older persons and a technology component tailored to the needs of the clients.

Several new elements might be added to future interventions. For example, technology for monitoring health conditions, and enhancing compliance and communication between health professionals and participants is available and can be incorporated in interventions for frail older persons. ${ }^{78}$ Techniques for enhancing self-management abilities in the older persons have been described in several studies $^{79,80}$ and seem applicable to, and promising for, community-dwelling frail older persons. A systematic approach towards enabling community-dwelling frail older persons to be engaged in meaningful social and productive activities might also be effective to prevent disability, as it fosters natural motivation and self-efficacy in the older persons. Recent studies show the potential of meaningful activities as a core of preventive programmes. ${ }^{81,82}$

It is likely that the development of interventions for community-living frail older persons has still some way to go. Further research focused on interventions that can prevent or delay disability in community-dwelling frail older persons is necessary.

\section{Acknowledgements}

We kindly thank mrs. A. Graat-Engelen for her support in retrieving publications. This review was funded by the Zuyd University of Applied Sciences. 


\section{References}

1. Aminzadeh F, Dalziel W, Molnar F. Targeting frail older adults for outpatient comprehensive geriatric assessment and management services: an overview of concepts and criteria. Rev Clin Gerontol. 2002;12(1):82-92.

2. Espinoza S, Walston JD. Frailty in older adults: insights and interventions. Cleve Clin J Med. 2005;72(12):1105-12.

3. Storey E, Thomas RL. Understanding and ameliorating frailty in the elderly. Topics in Geriatric Rehabilitation. 2004;20(1):4-13.

4. Hooi WC, Bergman H. A review on models and perspectives on frailty in older persons. SGH Proceedings. 2005;14(2).

5. Markle-Reid M, Browne G. Conceptualizations of frailty in relation to older adults. J Adv Nurs. 2003;44(1):58-68.

6. Slaets JP. Vulnerability in the elderly: frailty. Med Clin North Am. 2006;90(4):593-601.

7. Hogan DB, MacKnight C, Bergman H. Models, definitions, and criteria of frailty. Aging Clin Exp Res. 2003;15(3 Suppl):1-29.

8. Lally F, Crome P. Understanding frailty. Postgrad Med J. 2007;83(975):16-20.

9. Levers MJ, Estabrooks CA, Ross Kerr JC. Factors contributing to frailty: literature review. J Adv Nurs. 2006;56(3):282-91.

10. Aminzadeh F, Byszewski A, Dalziel WB, Wilson M, Deane N, Papahariss-Wright S. Effectiveness of outpatient geriatric assessment programs: exploring caregiver needs, goals, and outcomes. J Gerontol Nurs. 2005;31(12):19-25.

11. Jette AM. Toward a common language for function, disability, and health. Phys Ther. 2006;86(5):726-34.

12. Ferrucci L, Guralnik JM, Studenski S, Fried LP, Cutler GB, Jr., Walston JD. Designing randomized, controlled trials aimed at preventing or delaying functional decline and disability in frail, older persons: a consensus report. J Am Geriatr Soc. 2004;52(4):625-34.

13. Cutler DM. Declining disability among the elderly. Health Affairs. 2001;20(6):11-27.

14. Wieland D. The effectiveness and costs of comprehensive geriatric evaluation and management. Crit Rev Oncol Hematol. 2003;48(2):227-37.

15. Bours GJ, Ketelaars CA, Frederiks CM, Abu-Saad HH, Wouters EF. The effects of aftercare on chronic patients and frail elderly patients when discharged from hospital: a systematic review. J Adv Nurs. 1998;27(5):1076-86.

16. Mason A, Weatherly H, Spilsbury K, Arksey H, Golder S, Adamson J, et al. A systematic review of the effectiveness and cost-effectiveness of different models of community-based respite care for frail older people and their carers. Health Technol Assess. 2007;11(15):1-157, iii.

17. Avlund K. Disability in old age. Longitudinal population-based studies of the disablement process. Dan Med Bull. 2004;51(4):315-49.

18. Rubenstein LZ, Siu AL, Wieland D. Comprehensive geriatric assessment: toward understanding its efficacy. Aging (Milano). 1989;1(2):87-98.

19. McCusker J, Verdon J, Tousignant P, de Courval LP, Dendukuri N, Belzile E. Rapid emergency department intervention for older people reduces risk of functional decline: results of a multicenter randomized trial. J Am Geriatr Soc. 2001;49(10):1272-81.

20. Naylor MD, Brooten D, Campbell R, Jacobsen BS, Mezey MD, Pauly MV, et al. Comprehensive discharge planning and home follow-up of hospitalized elders: a randomized clinical trial. Jama. 1999;281(7):613-20.

21. Siu AL, Kravitz RL, Keeler E, Hemmerling K, Kington R, Davis JW, et al. Postdischarge geriatric assessment of hospitalized frail elderly patients. Arch Intern Med. 1996;156(1):76-81.

22. Hughes SL, Cummings J, Weaver F, Manheim LM, Conrad KJ, Nash K. A randomized trial of Veterans Administration home care for severely disabled veterans. Med Care. 1990;28(2):135-45. 
23. Burns R, Nichols LO, Graney MJ, Cloar FT. Impact of continued geriatric outpatient management on health outcomes of older veterans. Arch Intern Med. 1995;155(12):1313-8.

24. Burns R, Nichols LO, Martindale-Adams J, Graney MJ. Interdisciplinary geriatric primary care evaluation and management: two-year outcomes. J Am Geriatr Soc. 2000;48(1):8-13.

25. Oktay JS, Volland PJ. Post-hospital support program for the frail elderly and their caregivers: a quasiexperimental evaluation. Am J Public Health. 1990;80(1):39-46.

26. Rubin CD, Sizemore MT, Loftis PA, de Mola NL. A randomized, controlled trial of outpatient geriatric evaluation and management in a large public hospital. J Am Geriatr Soc. 1993;41(10):1023-8.

27. Hebert R, Robichaud L, Roy PM, Bravo G, Voyer L. Efficacy of a nurse-led multidimensional preventive programme for older people at risk of functional decline. A randomized controlled trial. Age Ageing. 2001;30(2):147-53.

28. Robichaud L, Hebert R, Roy PM, Roy C. A preventive program for community-dwelling elderly at risk of functional decline: a pilot study. Arch Gerontol Geriatr. 2000;30(1):73-84.

29. Silverman M, Musa D, Martin DC, Lave JR, Adams J, Ricci EM. Evaluation of outpatient geriatric assessment: a randomized multi-site trial. J Am Geriatr Soc. 1995;43(7):733-40.

30. Reuben DB, Frank JC, Hirsch SH, McGuigan KA, Maly RC. A randomized clinical trial of outpatient comprehensive geriatric assessment coupled with an intervention to increase adherence to recommendations. J Am Geriatr Soc. 1999;47(3):269-76.

31. Engelhardt JB, Toseland RW, O'Donnell JC, Richie JT, Jue D, Banks S. The effectiveness and efficiency of outpatient geriatric evaluation and management. J Am Geriatr Soc. 1996;44(7):847-56.

32. Toseland RW, O'Donnell JC, Engelhardt JB, Hendler SA, Richie JT, Jue D. Outpatient geriatric evaluation and management. Results of a randomized trial. Med Care. 1996;34(6):624-40.

33. Bernabei R, Landi F, Gambassi G, Sgadari A, Zuccala G, Mor V, et al. Randomised trial of impact of model of integrated care and case management for older people living in the community. Bmj. 1998;316(7141):1348-51.

34. Gagnon AJ, Schein C, McVey L, Bergman H. Randomized controlled trial of nurse case management of frail older people. J Am Geriatr Soc. 1999;47(9):1118-24.

35. Landi F, Onder G, Tua E, Carrara B, Zuccala G, Gambassi G, et al. Impact of a new assessment system, the MDS-HC, on function and hospitalization of homebound older people: a controlled clinical trial. J Am Geriatr Soc. 2001;49(10):1288-93.

36. Markle-Reid M, Weir R, Browne G, Roberts J, Gafni A, Henderson S. Health promotion for frail older home care clients. J Adv Nurs. 2006;54(3):381-95.

37. Melin AL, Bygren LO. Efficacy of the rehabilitation of elderly primary health care patients after shortstay hospital treatment. Med Care. 1992;30(11):1004-15.

38. Melis RJ, van Eijken MI, Teerenstra S, van Achterberg T, Parker SG, Borm GF, et al. A randomized study of a multidisciplinary program to intervene on geriatric syndromes in vulnerable older people who live at home (Dutch EASYcare Study). J Gerontol A Biol Sci Med Sci. 2008;63(3):283-90.

39. Phelan EA, Williams B, Penninx BW, LoGerfo JP, Leveille SG. Activities of daily living function and disability in older adults in a randomized trial of the health enhancement program. J Gerontol A Biol Sci Med Sci. 2004;59(8):838-43.

40. Rubenstein LZ, Alessi CA, Josephson KR, Trinidad Hoyl M, Harker JO, Pietruszka FM. A randomized trial of a screening, case finding, and referral system for older veterans in primary care. J Am Geriatr Soc. 2007;55(2):166-74.

41. Rockwood K, Stadnyk K, Carver D, MacPherson KM, Beanlands HE, Powell C, et al. A clinimetric evaluation of specialized geriatric care for rural dwelling, frail older people. J Am Geriatr Soc. 2000;48(9):1080-5.

42. Williams ME, Williams TF, Zimmer JG, Hall WJ, Podgorski CA. How does the team approach to outpatient geriatric evaluation compare with traditional care: a report of a randomized controlled trial. J Am Geriatr Soc. 1987;35(12):1071-8. 
43. Gitlin LN, Winter L, Dennis MP, Corcoran M, Schinfeld S, Hauck WW. A randomized trial of a multicomponent home intervention to reduce functional difficulties in older adults. J Am Geriatr Soc. 2006;54(5):809-16.

44. Cohen HJ, Feussner JR, Weinberger M, Carnes M, Hamdy RC, Hsieh F, et al. A controlled trial of inpatient and outpatient geriatric evaluation and management. N Engl J Med. 2002;346(12):905-12.

45. Boult C, Boult LB, Morishita L, Dowd B, Kane RL, Urdangarin CF. A randomized clinical trial of outpatient geriatric evaluation and management. J Am Geriatr Soc. 2001;49(4):351-9.

46. Gitlin LN, Hauck WW, Winter L, Dennis MP, Schulz R. Effect of an in-home occupational and physical therapy intervention on reducing mortality in functionally vulnerable older people: preliminary findings. J Am Geriatr Soc. 2006;54(6):950-5.

47. Phelan EA, Anderson LA, LaCroix AZ, Larson EB. Older adults' views of "successful aging"--how do they compare with researchers' definitions? J Am Geriatr Soc. 2004;52(2):211-6.

48. Chandler JM, Duncan PW, Kochersberger G, Studenski S. Is lower extremity strength gain associated with improvement in physical performance and disability in frail, community-dwelling elders? Arch Phys Med Rehabil. 1998;79(1):24-30.

49. Boshuizen HC, Stemmerik L, Westhoff MH, Hopman-Rock M. The effects of physical therapists' guidance on improvement in a strength-training program for the frail elderly. J Aging Phys Act. 2005;13(1):5-22.

50. Jette AM, Lachman M, Giorgetti MM, Assmann SF, Harris BA, Levenson C, et al. Exercise--it's never too late: the strong-for-life program. Am J Public Health. 1999;89(1):66-72.

51. Latham NK, Anderson CS, Lee A, Bennett DA, Moseley A, Cameron ID. A randomized, controlled trial of quadriceps resistance exercise and vitamin $D$ in frail older people: the Frailty Interventions Trial in Elderly Subjects (FITNESS). J Am Geriatr Soc. 2003;51(3):291-9.

52. Ota A, Yasuda N, Horikawa S, Fujimura T, Ohara H. Differential effects of power rehabilitation on physical performance and higher-level functional capacity among community-dwelling older adults with a slight degree of frailty. J Epidemiol. 2007;17(2):61-7.

53. Chin A Paw MJ, de Jong N, Schouten EG, Hiddink GJ, Kok FJ. Physical exercise and/or enriched foods for functional improvement in frail, independently living elderly: a randomized controlled trial. Arch Phys Med Rehabil. 2001;82(6):811-7.

54. Gill TM, Baker DI, Gottschalk M, Peduzzi PN, Allore H, Byers A. A program to prevent functional decline in physically frail, elderly persons who live at home. N Engl J Med. 2002;347(14):1068-74.

55. King MB, Whipple RH, Gruman CA, Judge JO, Schmidt JA, Wolfson LI. The Performance Enhancement Project: improving physical performance in older persons. Arch Phys Med Rehabil. 2002;83(8):10609.

56. Worm CH, Vad E, Puggaard L, Stovring H, Lauritsen J, Kragstrup J. Effects of a multicomponent exercise program on functional ability in community-dwelling frail older adults. Journal of the aging and physical activity. 2001;(9):414-24.

57. Timonen L, Rantanen T, Makinen E, Timonen TE, Tormakangas T, Sulkava R. Effects of a group-based exercise program on functional abilities in frail older women after hospital discharge. Aging Clin Exp Res. 2006;18(1):50-6.

58. Binder EF, Schechtman KB, Ehsani AA, Steger-May K, Brown M, Sinacore DR, et al. Effects of exercise training on frailty in community-dwelling older adults: results of a randomized, controlled trial. J Am Geriatr Soc. 2002;50(12):1921-8.

59. Luukinen H, Lehtola S, Jokelainen J, Vaananen-Sainio R, Lotvonen S, Koistinen P. Prevention of disability by exercise among the elderly: a population-based, randomized, controlled trial. Scand J Prim Health Care. 2006;24(4):199-205.

60. Latham NK, Bennett DA, Stretton CM, Anderson CS. Systematic review of progressive resistance strength training in older adults. J Gerontol A Biol Sci Med Sci. 2004;59(1):48-61.

61. Payette H, Boutier V, Coulombe C, Gray-Donald K. Benefits of nutritional supplementation in freeliving, frail, undernourished elderly people: a prospective randomized community trial. J Am Diet Assoc. 2002;102(8):1088-95. 
62. Kretser AJ, Voss T, Kerr WW, Cavadini C, Friedmann J. Effects of two models of nutritional intervention on homebound older adults at nutritional risk. J Am Diet Assoc. 2003;103(3):329-36.

63. Chin APMJ, de Jong N, Schouten EG, Hiddink GJ, Kok FJ. Physical exercise and/or enriched foods for functional improvement in frail, independently living elderly: a randomized controlled trial. Arch Phys Med Rehabil. 2001;82(6):811-7.

64. Kretser AJ, Voss T, Kerr WW, Cavadini C, Friedmann J. Effects of two models of nutritional intervention on homebound older adults at nutritional risk. Journal of the American Dietetic Association. 2003;103(3):329-36.

65. Mann WC, Ottenbacher KJ, Fraas L, Tomita M, Granger CV. Effectiveness of assistive technology and environmental interventions in maintaining independence and reducing home care costs for the frail elderly. A randomized controlled trial. Arch Fam Med. 1999;8(3):210-7.

66. Tomita MR, Mann WC, Santon K, Tomita AD, Sundar V. Use of currently available smart home technology by frail elders: process and outcomes. Topics in Geriatric Rehabilitation. 2007;23(1):24-34.

67. Tomita MR, Mann WC, Stanton K, Tomita AD, Sundar V. Use of currently available smart home technology for frail elders; process and outcomes. Topics in geriatric rehabilitation. 2007;23(1):2434.

68. Giannini R, Petazzoni E, Savorani G, Galletti L, Piscaglia F, Licastro F, et al. Outcomes from a program of home care attendance in very frail elderly subjects. Arch Gerontol Geriatr. 2007;44(2):95-103.

69. Beck A, Scott J, Williams P, Robertson B, Jackson D, Gade G, et al. A randomized trial of group outpatient visits for chronically ill older HMO members: the Cooperative Health Care Clinic. J Am Geriatr Soc. 1997;45(5):543-9.

70. Scott JC, Conner DA, Venohr I, Gade G, McKenzie M, Kramer AM, et al. Effectiveness of a group outpatient visit model for chronically ill older health maintenance organization members: a 2-year randomized trial of the cooperative health care clinic. J Am Geriatr Soc. 2004;52(9):1463-70.

71. Ollonqvist K, Aaltonen T, Karppi SL, Hinkka K, Pontinen S. Network-based rehabilitation increases formal support of frail elderly home-dwelling persons in Finland: randomised controlled trial. Health Soc Care Community. 2008;16(2):115-25.

72. Liang $\mathrm{MH}$, Partridge AJ, Gall V, Taylor J. Evaluation of a rehabilitation component of home care for homebound elderly. Am J Prev Med. 1986;2(1):30-4.

73. Beswick AD, Rees K, Dieppe P, Ayis S, Gooberman-Hill R, Horwood J, et al. Complex interventions to improve physical function and maintain independent living in elderly people: a systematic review and meta-analysis. Lancet. 2008;371(9614):725-35.

74. Wieland D, Hirth V. Comprehensive geriatric assessment. Cancer Control. 2003;10(6):454-62.

75. Stuck AE, Walthert JM, Nikolaus T, Bula CJ, Hohmann C, Beck JC. Risk factors for functional status decline in community-living elderly people: a systematic literature review. Soc Sci Med. 1999;48(4):445-69.

76. Femia $\mathrm{EE}, \mathrm{Zarit} \mathrm{SH}$, Johansson $\mathrm{B}$. The disablement process in very late life: a study of the oldest-old in Sweden. J Gerontol B Psychol Sci Soc Sci. 2001;56(1):P12-23.

77. Gezondheidsraad. Preventie bij ouderen: Focus op zelfredzaamheid. In: Gezondheidsraad, editor. Den Haag; 2009.

78. Botsis T, Hartvigsen G. Current status and future perspectives in telecare for elderly people suffering from chronic diseases. J Telemed Telecare. 2008;14(4):195-203.

79. Frieswijk N, Steverink N, Buunk BP, Slaets JP. The effectiveness of a bibliotherapy in increasing the self-management ability of slightly to moderately frail older people. Patient Educ Couns. 2006;61(2):219-27.

80. Lamers F, Jonkers CC, Bosma H, Diederiks JP, van Eijk JT. Effectiveness and cost-effectiveness of a minimal psychological intervention to reduce non-severe depression in chronically ill elderly patients: the design of a randomised controlled trial [ISRCTN92331982]. BMC Public Health. 2006;6:161. 
81. Fried LP, Carlson MC, Freedman M, Frick KD, Glass TA, Hill J, et al. A social model for health promotion for an aging population: initial evidence on the Experience Corps model. J Urban Health. 2004;81(1):64-78.

82. Graff MJ, Vernooij-Dassen MJ, Thijssen M, Dekker J, Hoefnagels WH, Rikkert MG. Community based occupational therapy for patients with dementia and their care givers: randomised controlled trial. Bmj. 2006;333(7580):1196. 



\section{CHAPTER 7}

\section{Rehabilitation in Practice: A disability prevention programme for community-}

dwelling frail older persons

In Press:

Daniels R, van Rossum E, Metzelthin S, Sipers W, Habets H, Hobma S, van den Heuvel W, de Witte L. A disability prevention programme for community-dwelling frail older persons. Clinical Rehabilitation (2011) 


\begin{abstract}
Objective: This article describes and justifies a primary care interdisciplinary programme for community-dwelling frail older people aimed to prevent disability.

Background: Disability is a negative outcome of frailty among older persons. Policy reports and research studies emphasize the need for programmes to reduce disability progression. Between 2008 and 2010, we developed such a programme.

Development: Following the Intervention Mapping protocol, a research team and a multidisciplinary professional developed the programme. Literature reviews and an expert meeting led to identification of basic elements, theory based methods, and practical tools.
\end{abstract}

The programme: The general practitioner and the practice nurse comprise the core team that can be extended by other professionals such as the occupational- and physical therapist. The programme includes six steps: (1) screening, (2) assessment, (3) analysis and preliminary action plan, (4) agreement on an action plan, (5) execution of the action plan (toolbox parts), and (6) evaluation and follow-up. The main features are:

- identifying risks for developing disability and targeting risk factors using professional standards and the 5A Behavioural Change Model to support self management, and identifying problems in performing activities and enhancing meaningful activities based on the Model of Human Occupation.

- Screening, individual assessment, tailor-made and client-centred care, selfmanagement support, case management, and interdisciplinary cooperation are important principles in delivering the programme.

Discussion: The disability-prevention programme seems promising for addressing the needs of frail older people for independent living and for targeting risk factors. Its feasibility and effects are currently being tested in a randomised controlled trial. 


\section{Introduction}

The rising number of frail older people poses various challenges for the public health care system. ${ }^{1}$ Frail older persons are recognised as being at risk of adverse outcomes such as death, falls, hospitalisation, and institutionalisation. ${ }^{2}$ Disability, defined as experiencing difficulty in performing activities in any domain of life ${ }^{3}$, is another negative outcome of frailty in older persons. Being independent and participating in society have great value for older people. ${ }^{4}$

Supporting older persons to live independently and participate in society are key policy targets for the Dutch government. ${ }^{5}$ Disability prevention for older persons, in contrast to disease prevention, has recently been addressed by the Dutch Health Council $^{6}$ as function-oriented prevention. The Council emphasises the necessity for development and evaluation of tailor-made interventions that focus on promoting independent functioning in daily life for (vulnerable) older persons with an important role for primary care, screening of vulnerable groups, and multidisciplinary cooperation.

Beswick and colleagues ${ }^{7}$ found that complex interventions like community-based care can support older people to live independently, though there is no clear evidence yet that one specific format is better than another. Various recent studies on proactive home visits for vulnerable groups of older people in the Netherlands, for example, showed conflicting results. These home visits by nurses did not turn out to be effective ${ }^{8,9}$ or only modest short term positive effects were reported. ${ }^{10}$

A more powerful intervention in primary care is needed to address the needs of frail older persons and prevent or postpone further functional decline. Although the Dutch care system is characterized by its strong emphasis on primary care ${ }^{11}$, it still seems insufficiently equipped to address the needs of frail older persons and to prevent disability. ${ }^{6}$ The overall reactive approach and lack of collaboration between disciplines ${ }^{6,12}$ poses challenges in implementing complex interventions.

Between 2008 and 2010, we developed an interdisciplinary primary care disabilityprevention programme for frail older people. Between May 2009 and January 2010, a feasibility study was conducted. ${ }^{13}$ Further, we studied the validity of the postal screening instrument used in the programme to identify frail elderly. ${ }^{14,15}$ In March 2010, sponsored by the Dutch National Care for the Elderly Programme, a full evaluation of the effectiveness of the programme started. ${ }^{16}$ This article aims to describe and justify the programme based on our development work. 


\section{Justification of the program - the theory}

The development of the programme was based on the Intervention Mapping proto$\mathrm{col}^{17}$ for developing health promotion programmes. Two teams cooperated during development: a research team conducted (literature) studies and pilots to support decision making, and a multidisciplinary task group developed practical tools. The latter group consisted of professional experts such as a general practitioner, a nursing home physician, a geriatrician, a practice nurse, a geriatric clinical nurse specialist, a physical therapist, an occupational therapist, an expert in technology, and a researcher $(\mathrm{RD})$ as the coordinator.

\section{Defining frailty}

Although there is still debate on the definition of frailty, agreement exists on the core feature of frailty: an increased vulnerability to stressors due to impairments in multiple, inter-related systems that lead to decline in homeostatic reserve and resiliency. ${ }^{18}$ Some authors describe frailty from a physiological perspective ${ }^{19-24}$ also referred to as physical frailty, while others consider frailty as multifactorial in nature, taking physical, psychological, social, and environmental factors into account. ${ }^{25}$

Literature on disability development in older people suggests that disability is multifactorial in nature. Stuck et al. ${ }^{26}$ identified in their review the following risk factors for developing disability in community-dwelling older persons: cognitive impairment, depression, comorbidity, increased and decreased body mass index, lower extremity functional limitation, falls, low frequency of social contact, and low level of physical activity. Femia et al. ${ }^{27}$ suggest that although disease conditions and physical impairments are as risk factors strongly related to an individual's functional abilities, other factors like the beliefs about one's health (e.g., subjective health), motivation, and self-efficacy are potentially as important.

With disability prevention as the main aim, a multifactorial approach to frailty was in our opinion a more promising approach in identifying and supporting communitydwelling frail older persons dealing with multiple diseases, problems, and risks. As a consequence, the programme takes a multifactorial perspective on screening, assessment, and treatment. As we consider frailty and disability as overlapping but distinct concepts, the programme needs to address the needs of frail elderly in different stages from mildly frail (without disabilities) to severely frail. 


\section{Programme outcome and goals}

The overall desired outcome of the programme is that frail older people can do those activities they need to do or enjoy doing. As the programme intends to be preventive in nature, two ways of supporting performance of activities were formulated.

- Identifying risks (as described by Stuck et al. ${ }^{26}$ ) for developing disability and targeting risk factors. Targets could be focused on cognitive impairment, depression, comorbidity, increased and decreased body mass index, lower extremity functional limitation, falls, low frequency of social contact, or low level of physical activity.

- Identifying problems in performing activities and enhancing meaningful activities. The Model of Human Occupation ${ }^{28}$ describes determinants influencing performance of activities. The model was successfully used in previous effect studies. ${ }^{29,} 30$ Goals could be focused on determinants such as personal causation (including self-efficacy), values, interests, roles, habits, performance capacity (mental and physical functions), skills (motor, cognitive, communicative), and the physical and social environment.

\section{Delivery of the programme}

Our literature review ${ }^{31}$ on interventions aimed at disability prevention in community-dwelling frail older persons concluded that a tailor-made, multidisciplinary and multifactorial approach, individualised assessment, and intervention, physical activity, assistive technology, case management, and long-term follow-up are promising elements for a disability-prevention programme. During an expert meeting (with 16 researchers in elderly care), experts recognised similar factors as self-management, a tailor-made and client-centred approach, involvement of the client system, and case management as relevant. Our programme elements are presented in table 1.

\section{Tailor-made care and self management support}

There is a strong body of evidence that self management support, using a mixture of components is effective in improving clinical outcomes. ${ }^{32}$ The $5 \mathrm{~A}$ Behavioural Change Model ${ }^{32,33}$ combines a client-centred approach, a model for behavioural change (Stages of Change model ${ }^{34}$ ), and motivational interviewing techniques ${ }^{35}$ to provide practical tools for professionals to support self-management. Rubak and colleagues ${ }^{36}$ reported that motivational interviewing outperforms traditional advice. The " 5 As" refer to assessing the older person's level of behaviour, beliefs, and motivation for change; advice adapted to the need for information; agreeing with the older person on a realistic set of goals and actions (goal setting and action planning); assisting to anticipate barriers and to increase skills; and arranging follow-up support. $^{32}$ Use of the $5 \mathrm{~A}$ Behavioural Model implies that goals and strategies to 
achieve the goals are individually determined and will depend strongly on the older persons' (self-perceived) problems, motivation, and capabilities. Self-management skills of the older person will influence whether goals are focused on the client or more on (support of) the social and physical environment.

Table 1 Elements of the disability-prevention programme

\begin{tabular}{|c|c|c|}
\hline General & Process & Intervention \\
\hline $\begin{array}{l}\text { Disease and function-oriented } \\
\text { - Tailor-made } \\
\text { - Client-centred } \\
\text { - Supporting self-management } \\
\text { - Multifactorial approach to frailty } \\
\text { - Multidisciplinary cooperation }\end{array}$ & $\begin{array}{l}\text { - Screening } \\
\text { - Assessment } \\
\text { - Case management } \\
\text { - Involvement of the client } \\
\text { system } \\
\text { - Multifactorial and flexible } \\
\text { - } \text { toolbox of interventions } \\
\text { - Long-term follow-up }\end{array}$ & $\begin{array}{l}\text { Determinants: } \\
\text { Risk factor for developing disabili- } \\
\text { ties, e.g., } \\
\text { - Falls } \\
\text { - Mood problems } \\
\text { - Lack of physical activity } \\
\text { Determinants } \\
\text { Enhancing performance of mean- } \\
\text { ingful activities } \\
\text { - Performance components (physi- } \\
\text { - Pal and mental functions) } \\
\text { - Values } \\
\text { - Interests } \\
\text { - Roles } \\
\text { - Habits } \\
\text { - Skills } \\
\text { - Social environment } \\
\text { - Physical environment } \\
\text { (e.g.,assistive technology) }\end{array}$ \\
\hline
\end{tabular}

\section{Meaningful activities}

As the programme aims to support older persons in continuing to do those activities they enjoy or need to do, meaningful activities had to be at the core of the programme. The Occupational Performance Process Model $^{37}$ was helpful in understanding that professionals need to explore concerns or problems of older persons with performance of activities, understand the older person's priorities, and use meaningful activities, where possible, as outcomes and means. The experience of doing can increase insight and beliefs in one's own capabilities (self-efficacy) ${ }^{28}$ which is central to self management. Graff et al. ${ }^{30}$ showed the effectiveness of a focus on meaningful activities in a programme for older persons with dementia.

\section{Interdisciplinary cooperation}

Besides having relevant clinical components, care programmes should also be seen as multidisciplinary protocols that encompass tasks, decision criteria, and work 
procedures for the care professionals involved. ${ }^{38}$ Studies into team collaboration in rehabilitation $^{39,40}$ show that a common care philosophy, defined roles, open and clear communication, regular structural communication, shared decision making, and goal setting enhance team cooperation.

\section{Delivering the programme - the practice}

The general practitioner and the practice nurse comprise the core team of the programme with the practice nurse in the role of case manager. This team can be extended to include the occupational therapist and physical therapist, other community care professionals, or hospital professionals. The programme consists of six steps (see also figure $1^{16}$ ).

\section{Step 1: Screening for frailty}

The Groningen Frailty Indicator (see appendix 1) accompanied by a covering letter from the general practice is sent to their population aged 70 or older. The Groningen Frailty Indicator ${ }^{41}$ is a fifteen-item screening instrument for determining the level of frailty. It focuses on the loss of functions and resources in four domains of functioning: physical (nine items), cognitive (one item), social (three items), and psychological (two items). Scores on the Groningen Frailty Indicator range from zero to fifteen. The instrument has shown high internal consistency and construct validity. $^{41,42}$ In our feasibility study ${ }^{13}$ among 41 elderly persons, nearly all participating elderly were willing to return the Groningen Frailty Indicator by post ( $90 \%$ response), and the number of missing items was low. The study into predictive values of three postal screening instruments ${ }^{15}$ showed that all three instruments, including the Groningen Frailty Indicator, do have potential to identify older persons at risk, but their predictive power is not sufficient yet. A substantial proportion of elderly identified as frail by the Groningen Frailty Indicator did not develop adverse outcomes in one year follow-up. For practice, this implies that additional information is needed to lower the number of false positives.

\section{Step 2: Assessment}

In the programme elderly with scores $\geq 5$ on the Groningen Frailty Indicator are considered as frail and are contacted by phone by the practice nurse to plan a home visit. During this visit, a multidimensional assessment is conducted by the practice nurse in the presence of the main informal caregiver (if available). This assessment focuses on:

- concerns and wishes expressed by the older person and informal caregiver

- risk factors for disability (based on Stuck et al. ${ }^{26}$ ), e.g., polypharmacy, mobility problems, falls, lack of physical activity, cognitive impairments, or mood problems 
- problems or concerns experienced in performing activities

- readiness to change

A structured assessment format is used, based on various tools derived from instruments that have proven their use in previous research, e.g., the 'Easy-Care assessment ${ }^{\prime 43}$ To optimise multidisciplinary communication, the format matches the structure of the electronic patient records of the geriatric department of the regional hospital.

Throughout the assessment, the practice nurse uses motivational interviewing techniques $^{35}$ to enhance collaborative partnership. Motivational Interviewing focuses on providing opportunities to help patients assess for themselves what might be important or possible and how change might be achieved. ${ }^{35}$ Reflective listening (accurately understanding a patient's story through open questions, reflections, gentle probing for more details, and use of summaries) is a key element for the practice nurse in conducting the multidimensional assessment. The Stages of Change model ${ }^{34}$ is used in monitoring the readiness to change.

The stages of change are:

- Precontemplation: not yet acknowledging that there is a problem that needs to be changed

- Contemplation : acknowledging that there is a problem but not yet ready or sure of wanting to make a change

- Preparation: getting ready to change

- Action: changing behavior

- Maintenance: maintaining the behavior change

- Relapse: returning to older behaviors and abandoning the new changes

\section{An example}

In answering the practice nurse's question about fall incidents, an older person tells that she recently fell three times, expresses her concern with these incidents, though doesn't seem to know what to change to improve the situation. The practice nurse concludes that, concerning fall incidents, the older person is in a contemplation phase ready to move to a preparation phase to receive information about possible actions to prevent falling.

In the end of the meeting, the practice nurse summarizes the answers of the older person (and informal caregiver) and validates outcomes by asking:

- "Could you tell me once more what are important activities for you to keep doing?" 
- "What would you say could support you in keep doing those activities as long as you wish?"

- "How motivated are you to take measures that will help you to keep doing those activities?"

Practice nurses were told to use the assessment in a flexible manner and to focus on understanding the story of the older person and not on problem-solving. The feasibility study ${ }^{13}$ showed that the assessment takes approximately one hour followed by half an hour of administration. The elderly were very positive about the opportunity to share their story and regarded the questions in the assessment relevant. Although professionals considered the assessment to contain sufficient items about activities, doubt was expressed about whether the older persons were sufficiently challenged to reflect on their patterns of activities in perspective of well-being and health. It is not common for elderly to express their problems and concerns in terms of activities, and more tools for professionals to discuss these kind of issues with elderly might be necessary.

Following assessment, the practice nurse and the general practitioner discuss whether additional assessments are needed. In case of problems or concerns about performing activities, a referral to the occupational therapist and physical therapist for an assessment is recommended to gain a better understanding of the underlying causes of experienced problems. Additional assessments by other specialised professionals follow, if needed (and if agreed on by the older person).

\section{Step 3: Analysis and preliminary action plan}

As complex care needs may arise, thorough analyses of data available from assessments is necessary before action takes place. The general practitioner and practice nurse discuss whether they can do the analysis and formulate a preliminary action plan together or whether the team has to be extended to include the occupational therapist and/or physical therapist and, other disciplines. In the case of an extended team, the professionals will hold a team meeting using a format based on the Model of Human Occupation. ${ }^{28}$ The older person and the informal caregiver can be invited to the team meeting.

The meeting takes a top-down approach towards analyses. After summarizing the older person's background and context, the team identifies the activities meaningful to this person, the problems or concerns the older person expressed in performing activities, and the risk factors for developing disabilities. This is followed by analyses in which concepts as personal causation (including self-efficacy), values, interests, roles, habits, performance capacity (mental and physical functions), skills (motor, 
cognitive, communicative), and the physical and social environment are taken into account.

After analyses, the team focuses on two questions:

- How can we support the older person to keep doing those activities that are meaningful?

- How can we support the older person to reduce the risk of developing disabilities in the near future?

The protocol offers specific information on a number of geriatric problems (e.g., dementia, falls, depression, incontinence) based on recent standards and guidelines that professionals can use in their deliberations (see also figure $1^{16}$ ). The best course of action is discussed in perspective of the older person's needs, readiness for change, and burden.

\section{An example}

A team meeting could result in several goals and actions:

- Lowering the risk of developing disability e.g.:

- Polypharmacy: Takes measures to increase safe use of medication (toolbox Stimulate health)

- Physical activity: Increases physical activity in daily life (toolbox Physical activity)

- Falls: Takes measures to reduce fall risks (toolbox Adapting the environment, skills or activities)

- Enhancing performance of (meaningful) activities e.g.:

- Older person wishes to continue working in the garden

- Physical environment: Uses aids and adaptations to facilitate performance of activities (toolbox Adapting the environment, skills or activities)

- Older person wishes to do meaningful activities in the weekend

- Interests: Is able to explore interests and to choose for and perform meaningful activities (toolbox Meaningful activities)

During development of the programme, some professionals, especially general practitioners, were reluctant towards these team meetings. The feasibility study showed some constraints in organising the meetings in one general practice. The twelve general practices in the randomised controlled trial, however, did, so far, not report problems with organization. Interdisciplinary meetings usually took one hour in which 3 or 4 older persons were discussed. Professionals experienced several advantages of this team collaboration, such as a more extensive picture of the older persons, sharing a mutual view on treatment, and a better understanding of the 
expertise of other disciplines. The feasibility study showed that education and guidance of teams may be important factors in achieving effective team collaboration.

\section{Step 4: Agreement on action plan}

The next phase relates to goal setting and action planning together with the older person (and informal caregiver). During a second home visit, the practice nurse explains that she would like to talk about measures to support the older person to keep doing meaningful activities. The practice nurse uses the motivational interviewing tool 'Agenda setting' to raise issues considered important by the older person, the informal caregiver and the team. In agenda setting, rather than impose the professionals' priority on patients, one conducts an overview by inviting patients to select an issue or behaviour that they are most ready and able to tackle, feeling free also to express one's own views. ${ }^{35}$

\section{An example}

Using the tool 'Agenda setting' the practice nurse asks:

"Is there some other topic that you would prefer to talk about? l'd like to talk at some point about your fear of falling and your wish to keep doing gardening. But, what makes sense to you right now?"

After an agenda is set, the older person's topics are discussed, followed by the topics of the practice nurse. Information and advice adapted to the needs of the older person (e.g. about possible treatment options) is given after permission was asked. Further, the practice nurse has the possibility to use motivational interviewing techniques as 'reflecting on pros and cons of change' (with the decision balance tool) and 'assessing importance and confidence in change' (with the importanceconfidence ruler; see appendix 2).

The outcome of this meeting is a list of goals, actions, and responsibilities that should meet the older person's and informal caregiver's needs. It is important for practice nurses to make time for this meeting, and do this as a face to face interaction and not over the phone. The practice nurse needs to involve the older person in decision making, and goals of treatment should be specific so that they motivate and direct a person's attention toward goal-relevant activities. It is not important that all issues seen as important by the team are negotiated. The setting of goals and actions is a part of a process in which professionals and clients establish a cooperation, in which a learning process begins leading to new insights and possibly to new goals and actions. Practice nurses in the feasibility study experienced that goal setting in this programme differs from goal setting with clients with COPD or diabe- 
tes. They reported that client goals in our programme range further in scope and are less pre-described.

\section{5: Executing the action plan}

A flexible toolbox of interventions is available to execute the action plan. For each toolbox part, a rationale and method are described to guide professionals. The toolbox exists of 5 parts:

\section{Meaningful activities (occupational therapist)}

This part of the toolbox is meant for older persons who lack confidence in capabilities or experience a lack in activities that they enjoy to do or that give meaning to life. The objective is to explore capacities, interests, and satisfaction with (performing) meaningful activities to be able to choose and perform meaningful activities. The method is based on occupational therapy literature ${ }^{28}$, motivational interviewing $^{35}$ and the Stages of Change model ${ }^{34}$ (see box 1 ).

\section{Adapting the environment, activities, or skills (occupational and physical therapist)}

This module is for older persons who experience problems in performance of activities. The objective is to adapt the environment, activities, or skills to enhance performance of activities. The method is based on standards and guidelines in occupational and physical therapy for advising assistive technology and strategy training. A functional exercise program (based on Gill et al. ${ }^{44}$ ) focusing on physical parameters as strength, balance, endurance, and flexibility is also part of this module.

\section{Social network and social activities (practice nurse)}

This part of the toolbox is for older persons with a small or instable network, without sufficient support from their network, experiencing tension in their social network, or loneliness. The objective is to strengthen the social network and/or increasing social activities. The method is based on the Dutch "Strengthening your network" programme, as described by Hofman et al. ${ }^{45}$ and the "Friendship course" by Stevens et al. ${ }^{46}$ Increased insight in one's network and wishes in relation to the social network is translated into goals and an action plan. 


\section{Daily physical activity (physical therapist)}

This part is meant for older persons aiming to increase daily physical activity. The method is based on motivational interviewing, stages of change model, and the seven-step approach as described by Resnick et al. ${ }^{47}$ (see box 2 )

\section{Stimulate health (general practitioner and practice nurse)}

This module is for older persons with chronic diseases or other risk factors for developing disabilities related to lifestyle. The objective is to take measures that stimulate health and a healthy lifestyle based on standards of the Dutch College of General Practitioners. In case of behavioral change, the general practitioner and practice nurse use motivational interviewing and the stages of change model to guide the older person.

During execution of the toolbox parts, the case manager (practice nurse) keeps in touch with the older person and the informal caregiver to monitor progress and satisfaction.

The feasibility study ${ }^{13}$ showed that professionals considered this toolbox appropriate for targeting frail elderly. The methods in the toolbox did request adaptation by professionals. They had to change their focus from problem-solving to support the client's self management and increase their own coaching skills.

\section{Step 6: Evaluation and follow-up}

After finishing parts of the toolbox, the practice nurse as case manager evaluates, with the older person, the achievement of goals, the implementation of strategies in daily life, and the need for support in the following period. This support could be arranged for example through regular visits, telephone, email, or community-based facilities. The professionals involved will be informed about the agreements. It is e.g. possible that an older person who was involved in the toolbox part "Physical Activity" still has a monthly telephone contact with the physical therapist to support the maintenance of new habits.

From the perspective of behavioural change, follow-up is a relevant phase. As a result of events and transitions, frail elderly can have more difficulty in maintaining self management strategies. In the feasibility study, arranging the follow-up was among the elements that were often not applied. Arranging a follow-up is a rather new element in primary care; it is not in the process of usual care and usually there is no reimbursement for it. A good system to organize who is responsible, for what, in which period, is necessary. This needs more attention in the programme. 


\section{Discussion}

This paper describes the content of a disability-prevention programme for community-dwelling frail older persons. The programme was designed to promote selfmanagement in a tailor-made way using motivational interviewing and meaningful activities as a vehicle to guide behavioural change. Literature studies provided relevant elements in delivering the programme. To shape the programme, the research team and the multidisciplinary task group used theories, models, and tools that have proven their usefulness in other contexts. How this combination works out in the context of community care targeted at frail older persons is still unclear. Bodenheimer $^{32}$, for instance, emphasizes that, although there is a strong body of evidence that self management support is effective in achieving clinical outcomes, much work is still needed on precising which activities are the most effective for which patients. Jonker et al. ${ }^{48}$ found in their review that a group based Chronic Disease Self Management Programme for vulnerable older persons (containing goal setting and action planning) was consistently beneficial for health behavior, especially with regard to the variables of exercise and self-care. How effective self management support is in an individual format in community-dwelling frail older persons is a question for further studies.

The combination of elements, theories, models, and tools has led to a complex intervention. New elements such as screening, motivational interviewing, meaningful activities in the core, team meetings, and a toolbox of interventions might be too ambitious for the average general practitioner's surgery. The feasibility study showed that the screening procedure needs reconsideration and that additional tools for professionals to support self management seem necessary.

\section{Acknowledgements}

For their contribution to developing the disability-prevention programme many thanks go to Inge Systermans, Margot van Melick, Frederique Prompers, Frank Vlaskamp, Henk Jochems, Rob de Ruijter and Michel Oostdam.

\section{Funding support}

This research was funded by Stichting Innovatie Alliantie and the Zuyd University of Applied Sciences. 


\section{References}

1. Hirdes JP. Addressing the health needs of frail elderly people: Ontario's experience with an integrated health information system. Age Ageing 2006; 35: 329-31.

2. Ferrucci L, Guralnik JM, Studenski S, et al. Designing randomized, controlled trials aimed at preventing or delaying functional decline and disability in frail, older persons: a consensus report. J Am Geriatr Soc 2004; 52: 625-34.

3. Jette AM. Toward a common language for function, disability, and health. Phys Ther 2006; 86: 72634.

4. Swedish National Institute of Public Health. Healthy Ageing: A challenge for Europe: Swedish National Institute of Public Health; 2007.

5. Ministerie van Volksgezondheid, Welzijn en Sport. Nota 'Ouderenbeleid in het perspectief van de vergrijzing'. Den Haag: Ministerie VWS; 2005.

6. Gezondheidsraad. Preventie bij ouderen: Focus op zelfredzaamheid. Den Haag: Gezondheidsraad; 2009.

7. Beswick $A D$, Rees $K$, Dieppe $P$, et al. Complex interventions to improve physical function and maintain independent living in elderly people: a systematic review and meta-analysis. Lancet 2008; 371: 725-35.

8. Nicolaides-Bouman A, van Rossum E, Kempen Gl, et al. Effects of home visits by home nurses to elderly people with health problems: design of a randomised clinical trial in the Netherlands [ISRCTN92017183]. BMC Health Serv Res 2004; 4: 35.

9. van Hout HP, Jansen AP, van Marwijk HW, et al. Prevention of adverse health trajectories in a vulnerable elderly population through nurse home visits: a randomized controlled trial [ISRCTN05358495]. J Gerontol A Biol Sci Med Sci 2010; 65: 734-42.

10. Melis RJ, van Eijken $\mathrm{MI}$, Teerenstra $\mathrm{S}$, et al. A randomized study of a multidisciplinary program to intervene on geriatric syndromes in vulnerable older people who live at home (Dutch EASYcare Study). J Gerontol A Biol Sci Med Sci 2008; 63: 283-90.

11. Knottnerus JA, ten Velden GH. Dutch doctors and their patients--effects of health care reform in the Netherlands. N Engl J Med 2007; 357: 2424-6.

12. Oosterbos van $\mathrm{H}$. The future of integrated primary care; community health centres at the heart of the neighbourhood. International Journal of Integrated Care 2006; 6: 1-2.

13. Daniels R, van Rossum E, van den Heuvel W, et al. Feasibility of an interdisciplinary programme to prevent disability in frail older persons. Submitted 2011.

14. Metzelthin SF, Daniels R, van Rossum E, et al. The psychometric properties of three self-report screening instruments for identifying frail older people living in the community BMC Public Health 2010; 10: 176

15. Daniels R, van Rossum E, Beurskens A, et al. The predictive validity of three self-report screening instruments for identifying frail older people in the community. Submitted 2011.

16. Metzelthin SF, van Rossum E, de Witte LP, et al. The reduction of disability in community-dwelling frail older people: design of a two-arm cluster randomized controlled trial. BMC Public Health 2010; 10: 511 .

17. Bartholomew LK, Parcel GS, Kok G, et al. Planning Health Promotion Programs: Intervention Mapping. San Francisco: Jossey-Bass; 2006.

18. Rockwood K. What would make a definition of frailty successful? Age Ageing 2005; 34: 432-4.

19. Fried LP, Ferrucci L, Darer J, et al. Untangling the concepts of disability, frailty, and comorbidity: implications for improved targeting and care. J Gerontol A Biol Sci Med Sci 2004; 59: 255-63.

20. Fried LP, Tangen CM, Walston J, et al. Frailty in older adults: evidence for a phenotype. J Gerontol $A$ Biol Sci Med Sci 2001; 56: M146-56.

21. Rockwood K, Fox RA, Stolee P, et al. Frailty in elderly people: an evolving concept. Cmaj 1994; 150: 489-95.

22. Walston J, Fried LP. Frailty and the older man. Med Clin North Am 1999; 83: 1173-94. 
23. Buchner DM, Wagner EH. Preventing Frail Health. Clinics in Geriatric Medicine 1992; 8: 1-17.

24. Fried LP. Frailty. In: Hazzard W.R. B, R.L. Blass, J.P., Ettinger, W.H., Halter, J.B., editor. Principles of Geriatric Medicine and Gerontology. 4th edn ed. New York, NY, USA: McGraw Hill; 1994. p. 1149-56.

25. Bergman H, Ferrucci L, Guralnik J, et al. Frailty: an emerging research and clinical paradigm--issues and controversies. J Gerontol A Biol Sci Med Sci 2007; 62: 731-7.

26. Stuck AE, Walthert JM, Nikolaus $T$, et al. Risk factors for functional status decline in community-living elderly people: a systematic literature review. Soc Sci Med 1999; 48: 445-69.

27. Femia $\mathrm{EE}, \mathrm{Zarit} \mathrm{SH}$, Johansson $\mathrm{B}$. The disablement process in very late life: a study of the oldest-old in Sweden. J Gerontol B Psychol Sci Soc Sci 2001; 56: P12-23.

28. Kielhofner $\mathrm{G}$, editor. Model of human occupation. Theory and application Baltimore: Wolters Kluwer/Lippincott Williams\&Wilkins; 2008.

29. Corr S, Bayer A. Occupational therapy for stroke patient after hospital discharge-a randomized controlled trial. Clinical rehabilitation 1995; 9: 291-6.

30. Graff MJ, Vernooij-Dassen MJ, Thijssen M, et al. Effects of community occupational therapy on quality of life, mood, and health status in dementia patients and their caregivers: a randomized controlled trial. J Gerontol A Biol Sci Med Sci 2007; 62: 1002-9.

31. Daniels R, Metzelthin SF, van Rossum E, et al. Interventions to prevent disability in frail communitydwelling older persons: an overview. European Journal of Ageing 2010; 7: 37-55.

32. Bodenheimer T, Grumbach K. Improving primary care. Strategies and tools for better practice. New York: Lange Medical Books/McGraw-Hill; 2007.

33. Glasgow RE, Emont S, Miller DC. Assessing delivery of the five 'As' for patient-centered counseling. Health Promot Int 2006; 21: 245-55.

34. Prochaska J, DiClemente C. Stages and processes of self-change in smoking: toward an integrative model of change. Journal of Consulting and Clinical Psychology 1983; 5: 390-5.

35. Miller WR, Rollnick S. Motivational Interviewing: preparing people for change. New York: Guilford Publications; 2002.

36. Rubak S, Sandbaek A, Lauritzen $T$, et al. Motivational interviewing: a systematic review and metaanalysis. Br J Gen Pract 2005; 55: 305-12.

37. Townsend E, Stanton S, editors. Enabling Occupation: an occupational therapy perspective. Ottawa: Canadian Association of Occupational Therapists; 2002.

38. Jansen $\mathrm{YJ}$, de Bont $A$, Foets $M$, et al. Tailoring intervention procedures to routine primary health care practice; an ethnographic process evaluation. BMC Health Serv Res 2007; 7: 125.

39. Gibbon B. An investigation of interprofessional collaboration in stroke rehabilitation team conferences. J Clin Nurs 1999; 8: 246-52.

40. Nijhuis BJ, Reinders-Messelink HA, de Blecourt AC, et al. A review of salient elements defining team collaboration in paediatric rehabilitation. Clin Rehabil 2007; 21: 195-211.

41. Steverink N, Slaets JPJ, Schuurmans H, et al. Measuring Frailty. Development and testing of the Groningen Frailty Indicator (GFI). Gerontologist 2001; 41: 236-7.

42. Metzelthin SF, Daniels R, van Rossum E, et al. The psychometric properties of three self-report screening instruments for identifying frail older people in the community. BMC Public Health 2010; 10: 176.

43. Richardson J. The Easy-Care assessment system and its appropriateness for older people. Nurs Older People 2001; 13: 17-9.

44. Gill TM, Baker DI, Gottschalk M, et al. A program to prevent functional decline in physically frail, elderly persons who live at home. N Engl J Med 2002; 347: 1068-74.

45. Hofman E, Scherpenzeel R. Netwerk rond cliënt versterken. Bunnik: Mezzo; 2008.

46. Stevens N, Albrecht H. De vriendschapscursus voor oudere vrouwen. Nijmegen: Radboud University/Centre of Psychogerontology; 1995.

47. Resnick B. A seven step approach to starting an exercise program for older adults. Patient Educ Couns 2000; 39: 243-52. 
48. Jonker AA, Comijs HC, Knipscheer KC, et al. Promotion of self-management in vulnerable older people: a narrative literature review of outcomes of the Chronic Disease Self-Management Program (CDSMP). Eur J Ageing 2009; 6: 303-14. 


\section{Annexus 1: The Groningen Frailty Indicator (GFI)}

\section{Physical components}

Are you able to carry out these tasks single-handedly and without any help? (The use of help resources such as a walking stick, walking frame or wheelchair is considered to be independent.)

1. Shopping

2. Walking around outside (around the house or to the neighbours)

3. Dressing and undressing

4. Going to the toilet

5. What mark do you give yourself for physical fitness? (scale 0 to 10)

6. Do you experience problems in daily life due to poor vision?

7. Do you experience problems in daily life due to being hard of hearing?

8. During the last 6 months have you lost a lot of weight unwillingly?

( $3 \mathrm{~kg}$ in 1 month or $6 \mathrm{~kg}$ in 2 months)

9. Do you take 4 or more different types of medicine?

Cognitive component

10. Do you have any complaints about your memory?

Social component

11. Do you sometimes experience emptiness around yourself?

12. Do you sometimes miss people around yourself?

13. Do you sometimes feel abandoned?

Psychological component

14. Have you recently felt downhearted or sad?

15. Have you recently felt nervous or anxious?

Scoring:

Questions 1-4: Yes = 0; No = 1

Question 5: 0-6 = 1; 7-10 = 0

Questions 6-9: No = 0; Yes = 1

Question 10: No = 0; Sometimes = 0; Yes $=1$

Questions 11-15: No = 0; Sometimes $=1$; Yes $=1$ 
Annexus 2: Importance-Confidence Ruler

\section{Importance-Confidence Ruler}

ON A SCALE OF 1 TO 10 OF IMPORTANCE, HOW IMPORTANT IS IT FOR YOU TO CHANGE THIS BEHAVIOUR

NOT

EXTREMELY

IMPORTANT

IMPORTANT

$\begin{array}{lllllllllll}0 & 1 & 2 & 3 & 4 & 5 & 6 & 7 & 8 & 9 & 10\end{array}$

ON A SCALE OF 1 TO 10 OF CONFIDENCE, HOW CONFIDENT ARE YOU ABOUT CHANGING THIS BEHAVIOUR?

NOT

EXTREMELY

CONFIDENT

CONFIDENT

1

2

3

4

5

6

7

8

9

10 


\section{Box 1 Toolbox part: Meaningful Activities}

\section{TOOLBOX PART: MEANINGFUL ACTIVITIES}

Objective: exploring capacities, interests and satisfaction in/with (performing) meaningful activities to be able to choose for and perform meaningful activities

Based on: occupational therapy literature (e.g.Graff et al, 2007), motivational interviewing, stages of change model

Target group: older persons who lack confidence in capacities or experience a lack in activities that they enjoy to do or that give meaning to life

\section{Orientation phase}

- Analyze with the client, by use of the OPHI II interview as a narrative method, the life story to get insight in the believes, values, habits, roles, meaning of activities, problems, goals and selfefficacy.

- Clarify with the interview the satisfaction with the current lifestyle, personal goals of the older person and self-efficacy regarding change.

\section{Goalsetting}

- Based on the outcomes of the OPHI II interview work together with the older person on goal setting starting with the question "how can I achieve that ......." (e.g. how can I achieve that I am doing activities during the weekend that give pleasure).

- Support the older person in formulating goals.

\section{Action planning}

- Explore with the older person possible activities or solutions. See it as a creative process in which feasibility of solutions are not (yet) important.

- Summarize the possibilities and discuss them in terms of feasibility (capacities of the older person, environmental resources, finances).

- Support the older person in making choices for exploration of activities or actions (e.g. an older person interested in flower arranging could find out where courses are given and what it takes to do flower arranging). Take the older persons self management skills in consideration in decision-making about who is responsible for actions.

\section{Experiencing and reflecting on activities}

- Consider a phase of experiencing activities and reflection on the experience as an important step in the process of finding out what is meaningful.

- Reflect with the older person on experiences in performing (new) activities, discuss possible barriers in performance. If necessary adjust the plan.

- If the experience was according to expectation discuss what is necessary to maintain doing the activity and make together an action plan.

\section{Maintenance}

- Make arrangements for follow-up.

- Discuss coping with relapse.

Relapse

- Reassess motivation and barriers. 


\section{Box 2 Toolbox part: Physical Activity}

\section{TOOLBOX PART: PHYSICAL ACTIVITY}

Objective: increasing daily physical activity

Based on: motivational interviewing, stages of change model and the seven-step approach. ${ }^{44}$

Target group: older persons aiming to increase daily physical activity

\section{Orientation phase}

- Explore current physical activities (LAPAQ instrument), readiness for change, wishes regarding physical activity, self-efficacy and social support.

- Provide information about physical activity in accordance with the needs of the older person. Suggest use of the Personal Activity Measure to increase insight in daily physical activity.

- Suggest conducting a screening into the physical capabilities of the older person.

- Encourage evaluation of pros and cons of behaviour change (contemplation phase).

- Explore the kind of activities the older person is interested in and how they would fit in their daily life (preparation phase). Suggest the possibility to try out physical activities to explore preferences.

\section{Action phase}

- Support the older person in developing an action plan.

- Support in identifying and solving barriers.

- Encourage to take small steps in executing the action plan

- Support self-efficacy by giving positive feedback

- Suggest use of the Personal Activity Measure (PAM) to monitor progress.

\section{Maintenance}

- Plan for follow-up support

- Discuss coping with relapse

\section{Relapse}

- Reassess motivation and barriers 



\section{CHAPTER 8}

\section{Feasibility of an interdisciplinary programme to prevent disability in frail older persons}

This article has been submitted as:

Daniels R, van Rossum E, van den Heuvel W, de Witte L.

Feasibility of an interdisciplinary programme to prevent disability in frail older persons. (2011) 


\begin{abstract}

\section{Backgound}

Disability, defined as experienced difficulty in performing activities, is one of the adverse outcomes of frailty and preventing disability in frail older persons is a priority in geriatric care. An interdisciplinary disability prevention programme was developed to address the needs of community-dwelling frail older persons for independent living and performing meaningful activities. In this study the feasibility of the programme was tested.
\end{abstract}

\title{
Methods
}

Older persons who had scored 5 or higher on a screening instrument followed the programme including assessments and a flexible toolbox of interventions provided by a team comprised of a general practitioner, a practice nurse, an occupational therapist and a physical therapist.

The actual use of the programme was monitored by log books. Interviews were conducted to reveal the satisfaction of participants and the opinions of professionals concerning the deliverability and acceptability of the programme.

\section{Results}

Forty-one older persons and ten professionals took part in the study. The response on the screening questionnaire was high (90\%). On average professionals regarded the population as mildly frail. Most parts of the protocol have been followed. Deviations and non-use of protocol parts were related to time aspects and difficulties with application. Participants were very positive about the support they got. Professionals regard the program as acceptable and deliverable for frail older persons experiencing problems in ADL and/or IADL.

\section{Conclusions}

The interdisciplinary disability-prevention program is considered feasible by professionals for Dutch primary care, especially for frail older persons experiencing problems in ADL and/or IADL. Reimbursment of assessments and team meetings is an important condition for professionals. This study reveals that aspects of the programme such as screening and self management support need further consideration. Giving its complexity, education, personal feedback and coaching of teams are important factors for the implementation of the program. 


\section{Background}

Being independent and having the possibility to participate in society have great value for older people in Western society. ${ }^{1,2}$ Disability, defined as experienced difficulty in performing activities ${ }^{3}$, is one of the adverse outcomes of frailty and preventing disability in frail older persons is a priority in geriatric care. ${ }^{4}$ Recently, the Dutch Health Council ${ }^{5}$ emphasised the necessity for development and evaluation of multidisciplinary tailor-made interventions that focus on promoting independent functioning in daily life for (vulnerable) older persons with an emphasis on primary care. Although the GP is appointed a leading role in integrated elderly care ${ }^{6}$, the question is whether the GP is well enough equipped to fulfill this role. ${ }^{5}$ Currently, primary care seems insufficiently prepared to address the needs of the frail older persons and to prevent disability. ${ }^{5}$ The overall reactive approach and lack of collaboration between disciplines ${ }^{5,7}$ poses challenges in implementing complex interventions.

Beswick and colleagues ${ }^{8}$ found that complex interventions such as communitybased care can support older people to live independently, though there is no clear evidence yet that one specific format is better than another. Our review ${ }^{9}$ into disability-prevention programs showed that promising features for programs are: multidisciplinary and multifactorial, individualised assessment and intervention, case management, long-term follow-up, a physical exercise component and the use of technology. Based on these features, an interdisciplinary primary care programme was developed to prevent disability in community-dwelling frail older persons. Following the Intervention Mapping protocol, a research team and a multidisciplinary task group of primary care professionals collaborated to design the programme to be compatible with current practice, competencies of professionals and the financial structure of primary care services.

Frailty is by some authors ${ }^{4}$ described from a physiological perspective also referred to as physical frailty, whereas others consider frailty to be multifactorial in nature. ${ }^{10}$ With an eye on disability prevention, a multifactorial approach to frailty was seen as a more promising approach in identifying and supporting the community-dwelling frail older persons dealing with multiple diseases, problems and risks. As a consequence, the disability prevention programme takes a multifactorial perspective on screening, assessment and treatment. It provides tailor-made and client centered care, self management support, case management and interdisciplinary cooperation. The programme uses the needs of the frail older persons for independent living and meaningful activities as a vehicle to guide behavioural, occupational and environmental change. The GP and the practice nurse (PN) comprise the core team that can be extended with occupational and physical therapists and other professionals. 
We studied the feasibility of this programme. By feasibility we mean the degree in which the programme is deliverable and acceptable in practice and how satisfied users are. This was tested by analysing the actual use in daily practice, the experiences of frail older persons and the acceptability to professionals. Concerning the latter specific attention was paid to opinions of professionals regarding key elements of the programme: meaningful activities in the core, interdisciplinary cooperation and self management support.

\section{Methods}

\section{Study design and population}

Two GP offices in the South of the Netherlands took part in the study. From each office one GP and two PNs were involved, joined in each of the settings by an occupational therapist and physical therapist. All 10 professionals received the program protocol, 6 hours training in the program and a 4 hour workshop about motivational interviewing techniques. The principal investigator (R.D.) was present in the team meetings.

After approval of the Medical Ethics Committee of Maastricht University and Maastricht University Hospital, the screening instrument GFI (Groningen Frailty Indicator ${ }^{11}$ ) was sent to elderly aged 70 or older accompanied by a letter from the GP. Respondents scoring 5 or higher on the GFI were contacted by phone by the PN or GP. Following, the older persons received patient information and an informed consent form by post. Recruitment continued until 42 older persons agreed to participate in the study. Of these 41 could be included in the analyses. One person was excluded due to a misinterpretation of the GFI-score.

\section{Intervention}

The programme has two main features:

- identifying risks (based on the review by Stuck et al. ${ }^{12}$ ) for developing disability and targeting risk factors using professional standards and the $5 \mathrm{~A}$ Behavioral Change Model ${ }^{13}$ to support self management

- identifying problems in performing activities and enhancing meaningful activities based on the Model of Human Occupation ${ }^{14}$ 
The programme consists of six steps (see also Appendix: Steps of the programme).

\section{Step 1: Screening for frailty}

The Groningen Frailty Indicator (GFI) accompanied by a covering letter from the GP is sent to elderly aged 70 or older. The $\mathrm{GFI}^{11}$ is a 15 -item screening instrument for determining the level of frailty with nine physical items, one cognitive item, three social and two psychological items. Those scoring 5 or higher are considered frail.

\section{Step 2: Assessment}

A multidimensional assessment is conducted by the PN (if present, in presence of the informal caregiver). The PN uses throughout the assessment motivational interviewing techniques to enhance collaborative partnership. The assessment focuses on concerns and needs expressed by the older person and informal care giver, risk factors for disability (e.g. falls, mobility problems, cognitive impairments or mood problems), experienced problems or concerns in performing activities and readiness to change. Following, the PN and GP discuss whether additional assessments are needed. In case of problems in performing activities it is recommended to involve the occupational therapist (OT) and physical therapist (PT) in additional assessments.

\section{Step 3: Analyses and preliminary action plan}

After the assessment phase the GP and PN fill in a problem list and discuss whether they can formulate a preliminary action plan or whether a team meeting is necessary with the OT and/or PT and, if necessary, other disciplines. During team meetings, the team identifies which problems or concerns the older person expressed in performance of activities and what risk factors exist for developing disabilities. Next, the best course of action is discussed taking the older person's needs, concerns and readiness for change, into account. Determinants for change are personal causation (including self-efficacy), values, interests, roles, habits, performance capacity (mental and physical functions), skills (motor, cognitive, communicative) and the physical and social environment. The protocol offers information on geriatric problems (e.g., depression) (see also Appendix: Steps of the programme ${ }^{15}$ ) that professionals can use in their deliberations.

\section{Step 4: Agreement on action plan}

With the motivational interviewing tool "agenda setting" the PN raises issues considered important by the older person, the informal caregiver and the team. In (face to face) dialogue with the older person and informal caregiver a list of goals, actions and responsibilities is compiled. Goals could e.g. be related to the use of mobility aids to be able to have a coffee in the theater, to medication to control diabetes, to 
increasing daily physical activity, to environmental adaptations to prevent falls or the organization of social support in keep doing meaningful activities.

Step 5: Executing the action plan

A flexible toolbox of interventions is available to execute the action plan (see also Appendix: Steps of the programme). Each toolbox part follows the general principles of the 5 A Behavioural Model, has a focus on meaningful activities, and uses (evidence-based) standards of practice. During this phase of the plan the case manager (PN) monitors and discusses with the older person and informal caregiver the progress and adjustments needed.

Step 6: Evaluation and follow-up

After finishing parts of the toolbox, the PN evaluates the progress with the older person and the caregiver and discusses the need for support in the following period. This support could be arranged for example through regular visits, telephone, email or community-based facilities. It is e.g. possible that an older person who was involved in the toolbox part Physical Activity still has a monthly telephone contact with the physical therapist to support the maintenance of new habits.

\section{Data collection and data-analysis}

The assessment of the PN provided data about characteristics of the participants. Data on the response on the screening instrument, reasons to refuse to participate or to withdraw from the programme, and the actual use of the programme were gathered by the PN in a log book. The Clinical Frailty Scale ${ }^{16}$ was used by the PN and the GP to get an impression of the clinical judgement of frailty. Information on performance according to protocol and opinions about the programme were obtained by semi-structured interviews. Eight face to face interviews were held with participants about the first four steps of the programme. Eight other participants were interviewed about the overall programme after they had finished toolbox parts (random selection). Fourteen interviews in different combinations (team, couples or individual) were conducted with professionals. Quantitative data were analysed by means of descriptive statistics. Qualitative data were categorised until themes and patterns in the answers emerged. ${ }^{17}$ An overview of data collection procedures is presented in table 1. 
Table 1 Overview of data collection procedures

\begin{tabular}{|c|c|c|c|}
\hline & $\begin{array}{c}\text { Log book } \\
\text { PN }\end{array}$ & $\begin{array}{l}\text { Interviews } \\
\text { elderly }\end{array}$ & $\begin{array}{c}\text { Interviews } \\
\text { professionals }\end{array}$ \\
\hline Patient characteristics & $x$ & & \\
\hline \multicolumn{4}{|l|}{ Actual use in practice } \\
\hline Screening & $x$ & & \\
\hline Assessment & $x$ & & \\
\hline Preliminary action plan & $x$ & & \\
\hline Agreement on action plan & $x$ & & \\
\hline Execution of action plan & $x$ & & \\
\hline Evaluation and follow-up & $x$ & & \\
\hline \multicolumn{4}{|l|}{ Experiences of frail elderly } \\
\hline First four steps & & $x$ & \\
\hline Total program & & $x$ & \\
\hline \multicolumn{4}{|l|}{ Acceptability by professionals } \\
\hline Reasons for protocol deviations & & & $x$ \\
\hline \multicolumn{4}{|l|}{ Feasibility: } \\
\hline - General & & & $x$ \\
\hline - Self management & & & $x$ \\
\hline - Interdisciplinary cooperation & & & $x$ \\
\hline - Meaningful activities & & & $x$ \\
\hline Added value & & & $x$ \\
\hline
\end{tabular}

\section{Results}

Both practices are characterized by professionals highly motivated to deliver elderly care in a systematic way; the first practice had experiences in delivering pro-active elderly care. In both practices GP and PN have been working together for several years; there had been no structured cooperation in elderly care with the involved OTs and PTs. Table 2 presents characteristics of the 41 patients. The average age is 76. Thirty participants (73\%) were females. The mean score on the GFI and the clinical judgment of the GPs and PNs indicate that on average the population under study is mildly frail. 
Table 2 Patient characteristics

\begin{tabular}{lccc}
\hline & $\begin{array}{c}\text { First practice } \\
(\mathrm{n}=24)\end{array}$ & $\begin{array}{c}\text { Second practice } \\
(\mathrm{n}=17)\end{array}$ & $\begin{array}{c}\text { Total } \\
(\mathrm{n}=41)\end{array}$ \\
\hline Age & 77,6 & 81,5 & 79,2 \\
Male/female (\%) & $30 / 70$ & $24 / 76$ & $27 / 73$ \\
Withdrawn from program & 0 & 3 & 3 \\
GFI score (1-15, mean) & 6,5 & 6,7 & 6,6 \\
ADL-score & 18,9 & 17,5 & 18,3 \\
Bartel-index (0-20, mean) & & 20,4 & 21,8 \\
IADL-score & 22,7 & & 4,4 \\
Lawton\&Brody (9-27, mean) & 4,1 & 4,7 & \\
Clinical Frailty Scale & & & \\
Rockwood (1-7, mean) & & & \\
\hline
\end{tabular}

Note: the underlined score refers to the most favourable score

\section{1) Actual use in daily practice}

Table 3 presents information on the actual use of the programme and reasons for protocol non-use and deviations. Most parts of the protocol have been followed. Deviations and non-use of protocol parts concern the use of the problem list in step 3 , face to face meetings to discuss the action plan in step 5, the arrangement of follow-up in step 6 and the use of motivational interviewing techniques through the entire programme. Reasons were related to time aspects and difficulties with application. 
Table 3 Actual use of the program and reasons for non-use and deviations

Actual use

(log book and interviews)
Reasons for non-use and deviations (as expressed in interviews)

\section{Screening}

- Out of 100 questionnaires 90 were returned

- 41 older persons $(45,5 \%)$ scored $\geq 5$ and were considered frail

\section{Assessment}

PNs conducted assessments with all frail elderly (average time of one hour) and results were discussed with the GPs. The problem list was not used. Number of referrals differ between practices

- Referral to OT: total $10(2 / 24-8 / 17)$

- Referral to PT: total 20 (9/24-11/17)

- Referral to outpatient clinic: 3

- Referral to other disciplines: 6

\section{Preliminary action plan}

- Preliminary action plan was discussed by PN and GP, or in case of referrals in an interdisciplinary meeting with GP, PN, OT and PT.

- On average interdisciplinary team meetings lasted one hour (four cases average on agenda) and were scheduled within two weeks after the referrals.

- Number of team meetings differs between the two GP practices ( 2 and 4 ).

\section{Agreement on the action plan}

- Following the preliminary action plan, the PNs did contact all older persons to discuss the action plan.

- Frequently this was done by telephone without use of the tool "agenda-setting".

\section{Execution of the action plan}

All older persons involved in 1, 2 or 3 toolbox parts.

Use of toolbox parts:

- Meaningful activities: 2

- Adaptation of environment/ skills/activities: 8

- Physical activity: 7

- Social network and activity: 1

- Stimulate health: 38

\section{Evaluation and follow-up}

- Follow-up was not always clearly arranged
Non-use of problem list:

According to PNs;

- Takes too much time

- Preference for own style

Lesser referrals to OT and PT in first practice:

Reason according to all professionals

( $1^{\text {st }}$ practice);

-Many pre-frail (score 4 on Clinical

Frailty Scale ) older persons without

ADL/IADL problems

Lesser team meetings in first practice

Reason according to GP and PNs $1^{\text {st }}$

practice:

-Many pre-frail older persons with

controllable disease symptoms and

without ADL/IADL problems

Lesser face to face contacts:

PNs said face to face meetings;

- Are time consuming

-Are not necessary with uncomplicated issues or when wishes older person are in line with team perspective

Little use of "Meaningful activities" and Social network and activities"

Professionals stated that;

-The selection of pre-frail persons has lowered the usability of these toolbox parts

-Most elderly have strong social support in these small communities with family close by

Arrangement of follow-up

-Professionals stated they had too little awareness of the necessity of structured follow-up support. 


\section{2) Experiences of frail elderly}

Eight older persons, who were interviewed about the first four steps of the programme, expressed their satisfaction with the screening and assessments. They could share their concerns and felt acknowledged. The pro-active approach is appreciated and has been experienced as a "welcome check-up". Half of the interviewees find it difficult to name what had been agreed on with the PN concerning the action plan.

Eight other persons were interviewed about their experiences with the entire programme. Most of them did not recall details of the assessment and goal-setting phase and for some it was difficult to differentiate between the programme and other forms of care they received (currently or in the past). All eight respondents, however, were able to describe the goals and actions they have recently worked on in the programme and the benefits they experienced from it. These are:

- 'getting personal attention', referring to the strong involvement of the professionals, the time spent on them, the attention to their story and feelings of being understood.

- 'being supported by reaching goals' referring to the importance of working on meaningful aims, the relevance of being informed and receiving good instructions and the adaptation of the approach to a day to day situation.

- 'experiencing a safety net' expressing the experiences of the elderly in feeling secure with a team behind them, knowing that in times of decline there are people who understand their situation and can be contacted when necessary.

From the interviews it became apparent how the physical and social environment impacts on maintenance of (new) habits. E.g. long winter conditions obstructed many older persons in doing meaningful activities and being physically active.

\section{3) Acceptability by professionals}

Feasibility

\section{General}

Professionals fully support the features of the programme and regard the steps in the programme and each tool as useful to guide practice. Despite the low use of the toolbox parts "Meaningful activities" and "Social network and activity", all ten professionals considered the content of the toolbox adequate for targeting frail older people. The programme has time-consuming parts, however, such as the assessment by the PN and the team meeting with all professionals. In both GP practices doubts have been expressed about the stability of the GFI instrument. In the first practice professionals described many of the selected elderly as pre-frail (despite 
scores $\geq 5$ on the GFI). For those older persons the programme is considered as 'too heavy'. The feasibility of the programme is regarded optimal when dealing with frail older persons experiencing complex problems and disabilities and on the condition that extensive assessments and team meetings are reimbursed.

\section{Interdisciplinary cooperation}

All professionals pointed out the added value of interdisciplinary meetings. Cooperation between the PN and GP has been experienced as intense in both GP practices. Clarity about tasks and responsibilities is seen as an important condition for their cooperation. PNs stressed the relevance of having the GPs trusting their capabilities. Logistically interdisciplinary meetings, even between PN and GP (working in the same building), were considered as not easy to arrange. The limited interdisciplinary cooperation with the OT and PT in the first GP practice was not caused by logistic problems, but was seen as a consequence of the selection of many pre-frail elderly. In the other GP practice the amount of referrals to the OT and PT was higher. There was a need for interdisciplinary analyses and tuning as care was considered complex. In the interviews these professionals emphasized the learning process they had to go through as a team and the necessity of getting support in this process.

\section{Self management support}

The application of the $5 A^{\prime}$ 's allows for reflection on the support of self management. Assessments by the PN, with eye for the older person's own concerns, needs and readiness for change, were done with all 41 frail older persons. Professionals stated a conscious use of the stages of change model, in contrary to a more implicit use of motivational interviewing techniques. Difficulties were reported with these techniques with older persons in the pre-contemplation stage. The PNs and GPs stated that is not always easy to support older persons in expressing their preferences. Frequently older persons mentioned that "the GP knows best". Occasionally, the GP and PN choose for a more paternalistic approach, convincing the older person of taking certain measures. Considering advice and assistance in reaching goals, professionals explained how they provide tailor-made information and support. The frail older persons expressed their satisfaction with the assistance they got for reaching goals. Professionals are aware that lesser attention for face to face meetings with the older person to agree on the action plan and the arrangement of follow-up can lower the effectiveness of self management support.

\section{Meaningful activities}

In general, the professionals expressed satisfaction with the degree in which the protocol supports them to focus on meaningful activities. E.g. they stated that the procedure of the team meeting puts emphasis on activities in analyses and delibera- 
tions for intervention. In the first practice, the professionals explained that their focus on meaningful activities was lower than expected due to a substantial proportion of the older persons not experiencing problems in ADL, IADL or other activities. Although the assessment of the PN contains sufficient items about activities, doubt was expressed about whether the older persons are sufficiently challenged during the assessment to reflect on their patterns of activities in the light of well-being and health. PNs said that for the older persons it is not common to express their problems and concerns in terms of activities.

\section{Added value}

Professionals stated that the programme leads to a more complete picture of the older person and the role of the informal caretaker as a consequence of the multifactorial perspective on frailty and the focus on (meaningful) activities. With its emphasis on risk factors and performance of (meaningful) activities the protocol helps them to focus on prevention. Throughout the programme they experienced a focus on issues that are (truly) relevant to the older person (and the informal caretaker) and felt able to work collaboratively with them. Furthermore, professionals expressed that the protocol, guiding both process and content, clarifies each person's role (e.g., the PN as case manager) and is a necessity in complex elderly care. Despite positive changes seen in their clients, it is difficult for the professionals to say whether the intervention prevents disability in the long term.

\section{Discussion}

This study shows that the professionals are positive about the feasibility of our disability-prevention program for Dutch primary care. The programme provides professionals a useful structure for delivering prevention of disability and enhances interdisciplinary cooperation, a focus on meaningful activities and self management support. Professionals considered the programme as acceptable and deliverable for frail older persons dealing with complex problems and disabilities. There is willingness among the older persons to participate. Ninety percent of those who received the postal screening questionnaire returned it. All selected frail older persons were prepared to join the programme, only a few persons dropped out and the experiences of the frail older persons with the approach were positive.

The selection of a substantial proportion of mildly frail older persons and the setting of the study in two smaller towns may have had influence on the application of the programme. How the programme would have been experienced in large rural areas is not clear. A limitation of this study is the lack of objective observations of the 
actual use of the programme in practice. We depended on the information provided by the professionals and the older persons. The in-depth information from the interviews, however, did provide insight in several issues that need consideration.

Although reactions from professionals and frail older persons on the programme were generally positive, issues such as the experienced logistic and time problems and the screening procedure could be obstacles for implementing the programme. Frequently the score on the screening instrument GFI did not match the clinical judgment of frailty and the prevalence of frailty $(45,5 \%)$ seems high compared to results of other studies. ${ }^{18}$

The programme takes differences in level of self management capabilities between persons into consideration. Still, it was not expected that so many older persons could not recall details of the action plan. Agreeing on the action plan is an important stage in the self management approach fostering motivation and commitment. The high non-use, despite awareness of the importance, of face to face meetings for goal setting and action planning (as well as for arranging follow-up) provokes questions whether the experienced time problems were the only reasons. Educating the PNs as to the importance of face to face meetings to reach agreement might be crucial. Furthermore, it might be necessary to provide professionals with a list of critical actions that should be taken for the intervention to be regarded as valid.

Although professionals generally agreed that meaningful activities should form a key component of the intervention how this might be achieved was less clear. Education and coaching could support the GP and PN and help them recognize the value of meaningful activities in cases where no ADL or IADL problems are experienced by the older person. However, the study raises questions about the methods and skills needed to support older people in sharing concerns and problems. For instance, did the combining of questions relating to biomedical issues and activities of daily living confuse older persons and encourage them to focus on medical and physical difficulties? From the findings it can be concluded that a four hour workshop about motivational interviewing is not sufficient. Literature ${ }^{19}$ suggests that for the implementation of motivational interviewing techniques education and personalized feedback is requested. The same seems to count for implementing interdisciplinary cooperation, experienced as a learning process needing coaching.

In conclusion, the interdisciplinary disability-prevention program is considered feasible by professionals in Dutch primary care for frail older persons experiencing problems in ADL and/or IADL, on the condition that assessments and team meetings are reimbursed. This study also reveals that aspects of the programme as screening and self management support needs further consideration. Giving its complexity, 
education, personal feedback and coaching of teams are among important conditions for the implementation of the program.

In March 2010 a randomised controlled trial (ISRCTN31954692) began to evaluate the effectiveness of the programme. ${ }^{15}$

\section{Acknowledgement}

We wish to thank both interdisciplinary teams in Buchten and Elsloo for their contribution in this study. Further, many thanks go to Walther Sipers, Herbert Habets, Sjoerd Hobma and Silke Metzelthin for their support. This study was supported by Stichting Innovatie Alliantie and the Zuyd University of Applied Sciences. 


\section{References}

1. Puts MT, Shekary N, Widdershoven G, Heldens J, Lips P, Deeg DJ. What does quality of life mean to older frail and non-frail community-dwelling adults in the Netherlands? Qual Life Res. 2007;16(2):263-77.

2. Health SNIoP. Healthy Ageing: A challenge for Europe: Swedisch National Institute of Public Health; 2007. Contract.

3. Jette AM. Toward a common language for function, disability, and health. Phys Ther. 2006;86(5):726-34.

4. Ferrucci L, Guralnik JM, Studenski S, Fried LP, Cutler GB, Jr., Walston JD. Designing randomized, controlled trials aimed at preventing or delaying functional decline and disability in frail, older persons: a consensus report. J Am Geriatr Soc. 2004;52(4):625-34.

5. Gezondheidsraad. Preventie bij ouderen: Focus op zelfredzaamheid. In: Gezondheidsraad, editor. Den Haag; 2009.

6. Genootschap NH. Huisartsgeneeskunde voor ouderen. Utrecht: NHG; 2009. Contract.

7. Oosterbos van $\mathrm{H}$. The future of integrated primary care; community health centres at the heart of the neighbourhood. International Journal of Integrated Care. 2006;6:1-2.

8. Beswick AD, Rees K, Dieppe $P$, Ayis $S$, Gooberman-Hill R, Horwood J, et al. Complex interventions to improve physical function and maintain independent living in elderly people: a systematic review and meta-analysis. Lancet. 2008;371(9614):725-35.

9. Daniels R, Metzelthin SF, van Rossum E, de Witte L, Kempen GI, van den Heuvel W. Interventions to prevent disability in frail community-dwelling older persons: an overview. European Journal of Ageing. 2010;7(1):37-55.

10. Bergman H, Ferrucci L, Guralnik J, Hogan DB, Hummel S, Karunananthan S, et al. Frailty: an emerging research and clinical paradigm--issues and controversies. J Gerontol A Biol Sci Med Sci. 2007;62(7):731-7.

11. Steverink N, Slaets JPJ, Schuurmans H, Lis van M. Measuring Frailty. Development and testing of the Groningen Frailty Indicator (GFI). Gerontologist. 2001;41:236-7.

12. Stuck AE, Walthert JM, Nikolaus T, Bula CJ, Hohmann C, Beck JC. Risk factors for functional status decline in community-living elderly people: a systematic literature review. Soc Sci Med. 1999;48(4):445-69.

13. Bodenheimer T, Grumbach K. Improving primary care. Strategies and tools for better practice. New York: Lange Medical Books/McGraw-Hill; 2007.

14. Kielhofner G, editor. Model of human occupation. Theory and application Baltimore: Wolters Kluwer/Lippincott Williams\&Wilkins; 2008.

15. Metzelthin SF, van Rossum E, de Witte LP, Hendriks MR, Kempen GI. The reduction of disability in community-dwelling frail older people: design of a two-arm cluster randomized controlled trial. BMC Public Health. 2010;10(1):511.

16. Rockwood K, Song X, MacKnight C, Bergman H, Hogan DB, McDowell I, et al. A global clinical measure of fitness and frailty in elderly people. CMAJ. 2005;173(5):489-95.

17. Kvale $S$. An introduction to qualitative research interviewing. California: Thousand Oaks; 1996.

18. Schuurmans H, Steverink N, Lindenberg S, Frieswijk N, Slaets JP. Old or frail: what tells us more? J Gerontol A Biol Sci Med Sci. 2004;59(9):M962-5.

19. Lozano P, McPhillips HA, Hartzler B, Robertson AS, Runkle C, Scholz KA, et al. Randomized trial of teaching brief motivational interviewing to pediatric trainees to promote healthy behaviors in families. Arch Pediatr Adolesc Med164(6):561-6. 

CHAPTER 9

General Discussion 


\subsection{Introduction}

The main objective of this thesis was to develop an evidence-based and feasible disability-prevention programme for community-dwelling frail elderly. The research questions were:

- What is the state of art in the frailty literature concerning the concept of frailty and instruments for identifying frail elderly?

- What is the validity of postal screening questionnaires to identify communitydwelling frail elderly?

- What is known in the literature about effective elements of disability-prevention programmes and how can these be integrated into a comprehensive and coherent programme?

- To what extent is a new interdisciplinary disability-prevention programme for community-dwelling frail elderly feasible in Dutch primary care?

Between 2007 and 2011 several studies were conducted:

- Literature study into the state of art in the frailty literature concerning the concept of frailty and instruments for identifying frail elderly (question 1)

- Cross-sectional study into the construct validity and internal consistency of three postal screening questionnaires. (question 2)

- Longitudinal study into the predictive validity of these screening questionnaires. (question 2)

- Systematic review into the content, methodological quality and effectiveness of intervention studies for the prevention of disability in community-dwelling physically frail elderly. (question 3)

- Narrative review into the content of interventions targeted at disability prevention in community-dwelling frail older persons. (question 3)

- Development of an evidence based interdisciplinary disability-prevention programme for community-dwelling frail elderly. (question 3)

- Feasibility study into the interdisciplinary disability-prevention programme for community-dwelling frail elderly. (question 4)

This chapter summarizes the main findings of these studies, followed by some methodological and theoretical considerations. Finally, overall conclusions and implications for practice and future research are given. 


\subsection{Main findings}

\section{Literature on frailty concept}

Our literature study into the frailty concept clarified that no consensus yet exists on the definition of frailty. The debate has focused on whether frailty should be defined purely in terms of biomedical factors (addressed to as physical frailty) or other factors should be included as well. Related to it, the debate continues on appropriate indicators for frail older people and how to assess these. As a consequence, no golden standard is present for identifying frail elderly. Community-based intervention research in the field of frail elderly is relatively new. Overviews of these interventions are scarce and face difficulties due to a lack of consensus for an explicit conceptual frailty definition and validated practical screening criteria.

\section{Validity of the screening tools}

We first evaluated and compared the internal consistency and construct validity of three instruments to detect frail elderly, using a postal screening procedure: the Groningen Frailty Indicator (GFI), the Tilburg Frailty Indicator (TFI) and the Sherbrooke Postal Questionnaire (SPQ). A questionnaire was sent to nearly 700 community-dwelling older people ( $\geq 70$ years). The response rate was $77 \%$. Prevalence estimates of frailty ranged from $40 \%$ to $59 \%$. The GFI and TFI showed high internal consistency and construct validity in contrast to the SPQ.

Then the predictive values of the three screening tools were studied in this sample. We focused on the development of disabilities, mortality and hospital admissions over one year follow-up ( $n=430)$. Adjusted odds ratios showed that those identified as frail by any of the three instruments have more than twice the risk for developing disabilities compared to the non-frail group; those identified as frail by the TFI and SPQ have more than twice the risk of being admitted to a hospital. Diagnostic values of the three instruments are, however, insufficient. Sensitivity and specificity for development of disabilities, e.g., are between 60 and $80 \%$ and between 50 and $70 \%$ respectively. In a two-step screening approach, these instruments would select a large number of false positives, implying that many older persons would have to go through an unnecessary extensive additional assessment.

\section{Promising elements and development of the preventive programme}

Our systematic review on the content and effectiveness of disability-prevention programmes targeted at physically frail community-dwelling elderly showed no evidence for the effect of nutritional interventions on disability outcomes, nor for 
the effect of single lower extremity strength training. However, we found indications that relatively long-lasting and high-intensive multicomponent exercise programs have a positive effect on ADL and IADL disability for community-living moderate physically frail older persons.

A second review on interventions targeted at community-dwelling frail older persons (not only physically frail) provided insight in promising elements of the 49 interventions that had been tested so far. This narrative review showed that future interventions may be directed towards tailor-made, multidisciplinary and multifactorial interventions, with individualised assessment and interventions conducted by a (primary) care team, involving case management and long-term follow-up. These tailor-made programmes may include a physical exercise component for moderate physically frail older persons and a technology component as well.

These reviews and further literature studies led to identification of basic elements, theory based methods and practical tools. A research team and a multidisciplinary professional task group then developed a disability prevention programme. The programme aims to support frail older persons in performance of activities meaningful to them by targeting risk factors and problems in performing these activities. The general practitioner (GP) and practice nurse (PN) comprise the core team that can be extended by other professionals such as the occupational- and physical therapist. The programme includes six steps: (1) screening, (2) assessment, (3) analysis and preliminary action plan, (4) agreement on action plan, (5) execution of the action plan (toolbox parts), and (6) evaluation and follow-up. Screening and individual assessment, tailor-made and client-centred care, self-management support, case management and interdisciplinary cooperation are important principles of the programme. The programme includes a toolbox of five interventions that can be utilized in a flexible manner.

\section{Feasibility study}

Forty-one older persons and ten professionals took part in the feasibility study that monitored the actual use of the programme and experiences of frail elderly and professionals. Most parts of the protocol were followed. Parts of self management support (face to face meetings to discuss the action plan, the arrangement of follow-up and the use of motivational interviewing techniques) were often not applied according to protocol. Deviations and non-use of protocol parts were related to time constraints and difficulties in executing specific elements of the protocol in practice. The participants were very positive about the support they received; they appreciated the personal attention and the support for obtaining personal goals. 
Professionals regarded the programme as acceptable and deliverable for those experiencing problems in ADL and/or IADL. They stated that the programme led to a more comprehensive view on the older person and the role of the informal caregiver as a consequence of the multifactorial perspective on frailty and the focus on (meaningful) activities. Furthermore, professionals expressed that the protocol, guiding both process and content, clarified each person's role (e.g., the PN as case manager) and enhanced interdisciplinary cooperation. A substantial proportion of the selected elderly were pre-frail, according to the GP and PN. We concluded that professionals involved regarded the interdisciplinary disability-prevention programme as feasible for Dutch primary care. Several of its elements (e.g. screening and self management), though, need further attention.

\subsection{Methodological considerations}

\section{Validity of the screening tools}

The strengths of our 'validity studies' were the comparisons of the psychometric properties of three frailty instruments, the proven feasibility of the postal procedure, the high response rates and the completeness of data sets (e.g. the low number of missing data). How to operationalise development of disability and the timeframe for follow-up have been key issues in designing the prospective study. We chose to dichotomize development of disabilities as a change from independent performance of an activity into a dependent one, being a rather clear-cut difference. With this choice we did not analyze more subtle changes in performance of activities. Fried et al. ${ }^{1}$ described the concept of preclinical disability, arguing that self perception of early changes in performance of activities may represent a selfreported state of frailty. From that perspective, we might have conducted additional analyses on the association between frailty and subtle changes in performance of activities. In view of prevention, these early signs of disability (within a limited follow-up period) seem relevant.

Regarding follow-up, the timeframe of one year is, compared to other longitudinal frailty studies ${ }^{2,3}$, rather short. From a scientific perspective a longer follow-up would have been valuable to gain more insight in frailty and disability transitions over time. However, from a clinical (e.g. GP) perspective, one year might seem a reasonable timeframe for pro-active elderly care. Within this limited timeframe, still $24 \%$ of older persons did develop disabilities in our study. 


\section{Literature reviews}

Methodological challenges in conducting the reviews were the variety of definitions and criteria for frailty and defining disability as an outcome measure. At that time no guidelines for frailty reviews were available yet. Search terms regarding frail elderly were chosen based on frailty literature. We used search terms as frail*", "vulnerable", "at risk", "high risk", "low functioning", and MESH terms like "chronic disease" and "disabled persons" in combination with the MESH term "aged". This is much in line with the search terms the Canadian Initiative on Frailty and Ageing ${ }^{4}$ presented in October 2009 as a part of their methodology for conducting systematic reviews. With these search terms, we did not miss relevant articles from the last decade. There is a possibility, however, that 'older studies' were not identified.

How to use disability as an outcome measure was another issue. In our definition ${ }^{5}$, disability refers to experienced difficulty in performance of activities in any domain of life. As this is a rather broad definition, it was decided to limit disability to ADL and IADL measures, in line with many studies on aging ${ }^{6}$ and frailty. ${ }^{4}$ Although possible, we think that we did not miss 'key studies' that used other disability measures.

Despite the strict focus of the first review on studies that considered frailty as a physiological syndrome, the homogeneity of the study populations in 'frailty reviews' is doubtful. Various criteria for frailty render different selections of elderly people. ${ }^{7}$ Also, the reviews showed that disability was frequently not the primary outcome measure of the included studies. Due to small sample sizes trials may have been underpowered to detect differences on disability. Finally, studies in our narrative review were not assessed for their methodological quality; randomised and controlled clinical trials were considered as a (minimal) standard for quality. Although these limitations warrant for cautious interpretation of results, our primary goal of the reviews was to prone for promising intervention elements for our disability-prevention programme.

\section{Feasibility study}

The main aim of the feasibility study was to explore the strong and weak points of the programme in view of its feasibility. Our mixed methods approach - in-depth interviews and registration of use of the programme in log books - has been successful in revealing features and elements that need further consideration. These relate, e.g., to the screening procedure, the self management approach and environmental barriers. Still, a limitation of this study is the lack of objective observations of the actual use of the programme. We depended on the information provided by the professionals and older persons. As these measurements were not blinded, the programme may have been valued too positive by the respondents. 


\subsection{Theoretical considerations}

Two major pillars of the programme (and this thesis) need further reflection: our choices regarding (1) screening for frailty and (2) the development and content of the intervention part. Regarding the latter, we start with a reflection on our overall approach to the programme development. Then we reflect on some issues related to our approach towards self management support.

\section{Screening}

In developing the programme we chose for a postal screening procedure with the Groningen Frailty Indicator (GFI) as multifactorial screening tool. By then the GFI was the most familiar and frequently used instrument in the Dutch context and a pilot study showed its feasibility as postal questionnaire. ${ }^{8}$ The study into the predictive abilities of the GFI and other frailty instruments showed that these self-report instruments are not yet optimal. There are several issues that need consideration before implementing such tools.

First of all, with an ongoing debate on frailty domains, it is still unclear how to measure frailty validly. Some 'frailty authors ${ }^{\prime 9,10}$ perceive a consensus on a multifactorial approach to frailty including the domains of nutrition, physical activity, mobility, energy, strength, cognition and mood. In contrast, Abdellan et al. ${ }^{11}$ conclude in their review that recent research did not solve previous controversies on frailty; no clear consensus regarding frailty domains or screening instruments emerged.

Secondly, the effectiveness of postal screening in perspective of dynamics of frailty needs reconsideration. Frailty is characterized by frequent transitions between frailty states over time and often emerges or decreases after events such as a fall, disease, hospital admission or decease of a spouse. ${ }^{12,13}$ Performing an annual or biannual (postal) screening might miss those elderly with sudden increased risk or with other changes which may be clinically meaningful. Therefore, additional strategies to identify frail elderly are needed. Case finding, using clinical judgment during consultation, is an option in this respect. Rockwood et al. ${ }^{14}$ showed e.g. that clinical judgment with the Clinical Frailty Scale can yield relevant prognostic information.

Further it needs reconsideration how the advantages of self report measures relate to their disadvantages. Research into the appropriate domains for identifying frailty will continue and affect measurement methods. Some domains can be accurately assessed by means of self reports. For other domains performance based measures seem more appropriate. Hand grip strength and gait speed, e.g., have repeatedly been reported as relevant frailty criteria. ${ }^{15,}{ }^{16}$ Advantages of performance-based 
measurement of these physical functions, compared to self reports, include better reproducibility, greater sensitivity to change, and less vulnerability to external influences such as cognition, culture, language, and education. ${ }^{16}$ Technological developments create new possibilities for these measurements. Preliminary experiences are gained currently with monitoring frailty indicators such as physical activity, balance and grip strength at home, with electronic data transmission to relevant professionals involved (e.g. GP and physical therapist). ${ }^{17}$ These developments will enhance inclusion of performance based measures in screening and monitoring procedures.

\section{Disability-prevention programme}

\section{Approach to developing the programme}

Developing an evidence based and feasible disability-prevention programme directed to frail elderly in primary care was the aim of this thesis. Innovative projects face the challenge of keeping balance between initiation and implementation of these innovative improvements. ${ }^{18}$ Our project was no exception. To achieve a good combination of evidence, theoretical frameworks and a fit with current practice, we collaborated with care professionals and based the development process on the Intervention Mapping protocol. ${ }^{19}$

Joining an initiative of a regional organization for GPs and the geriatric department of the regional hospital led to collaboration between the research team and the multidisciplinary task group of care professionals responsible for developing practical tools. The research team performed literature studies and held an expert meeting in order to render an evidence based reference frame. Based on this frame, the multidisciplinary group was responsible for developing a protocol compatible with practice, respecting current roles and (financial) structures in primary care. In the multidisciplinary team, subgroups developed practical tools (e.g. assessment tools and toolbox parts). Concepts were discussed with the multidisciplinary task group and the research team.

There are several factors that determine whether adoption of an innovation will occur: compatibility, observability, relative advantage, trialability and complexity. ${ }^{20}$ Collaboration with care professionals has been beneficial for developing a programme compatible with the values, norms, and clinical practice in primary care (compatibility). Features of the programme such as pro-active screening, working interdisciplinary and self management are in line with recent Dutch policy reports of professional and governmental bodies. They are recognized by professionals as valuable innovations (observability) and confirm the important role of primary care in care for frail elderly (relative advantages). Further, the evidence based approach 
to development of the programme, the pilot and the feasibility study promotes faith that the evidence is correct. To a certain extent the programme is modifiable for professionals to specific situations and can be tried out on a limited base (trialability). However, complexity seems an issue in need of attention before implementation of the programme.

The feasibility study showed that some key elements were not applied as expected. It also showed some organizational and financial constraints. We may have underestimated environmental factors that needed innovative solutions and "out of the box" thinking. During the development process, additional actions as mentioned below would have given us a better view on barriers in delivering the programme and whether our approach matches the competencies of care professionals and the preferences of frail elderly.

\section{Exploring environmental constraints}

More emphasis in step 1 of Intervention Mapping on exploring environmental constraints in primary care would have led to better insights in barriers and the involvement of additional stakeholders. If working load and reimbursement are major factors in implementing the programme, health insurance companies should have been involved in the project.

\section{Testing materials more extensively}

Desk research has been a major pillar in our programme development. More action in the developmental phase by trying out ideas in practice and constant reflection might have led to solutions that would have improved changes in traditional ways of working. For instance, ICT could have enabled distance interdisciplinary meetings, saving travel time of professionals. The pretesting of materials could have been more extensive to fine-tune the approach.

\section{Organising user-group involvement}

Prior to the feasibility study, we might have involved frail elderly or representatives more actively in the development of the programme. Their participation could have helped in fine-tuning the self management support to the needs of the user group. Recent projects funded by the Dutch National Care for the Elderly Programme are obliged to involve the user group from the initiation phase. These projects might lead to more knowledge on how to involve the frail elderly themselves in programme development and whether non-frail elderly can represent the user group adequately. 


\section{Self management support}

Self management refers to daily activities that people do or not do to maintain an optimal health or well-being. ${ }^{21}$ Supporting self management of frail elderly to maintain performance of activities meaningful to them is a key element in our disabilityprevention programme. We assume that these activities enhance both health and wellbeing, and are protective for developing disabilities. Self-management skills of the older person will influence whether goals are focused on the client or more on (support of) the social and physical environment.

The support of self management by professionals includes provision of information, assistance in goal setting, action planning and reaching goals, and arrangement of follow-up support. ${ }^{22}$ This approach seems to appeal to frail elderly. In the feasibility study, all persons identified as frail were willing to join the programme, we had very low dropout rates and the participants were positive about the support they received for obtaining personal goals. Recruiting and engaging elders to participate in any type of health program and to complete such programs is considered challenging. ${ }^{23}$ Van Elzen et al. ${ }^{24}$ experienced that many Dutch frail elderly (also identified with the GFI) refused to participate in a self management group programme, and related this to barriers regarding physical mobility, travel distance, and social support. Our programme lacks these barriers as assessment and interventions take place in the older person's context (being a part of the self management approach). Furthermore, we think that participation is enhanced by the trust of people in their GP. In contrast to other self management programmes, our programma was delivered by GP practices.

Professionals did not always apply elements of self management support according to protocol. In interviews many elderly participants had difficulties in naming goals they were working on, and in making a distinction between the programme and other care they received. These findings do urge for some reconsideration of the approach, that heavily relies on collaboration in goal setting and action planning, emphasizing shared decision-making. Elderly might not be always receptive to this approach, or they may have cognitive problems. Further, we assume that as a result of events and transitions it is for frail elderly more difficult to maintain self management strategies. Frail elderly are more susceptible for disease and their decreased reserves makes them vulnerable for (changes in) the physical and social environment. Interviews in the feasibility study showed e.g. the impact of hospital admission of a spouse, waiting for home adaptations, and the burning down of the main shopping mall on routines and performance of activities of frail elderly. More knowledge is needed to optimize goal setting and action planning and arrangement of follow-up. 
The conclusions of the Dutch College of Health Care Professions and Education ${ }^{25}$, that a focus on self management requires more coaching competencies of primary care professionals, emphasizes the importance of providing professionals with adequate tools for self management support. In our programme, guiding clients in reflecting on their daily activities and defining problems and needs towards (meaningful) activities challenged the communicative skills of professionals. Graff et al. ${ }^{26}$ showed that by using an occupational therapy tool, it is possible to set (in collaboration) goals for meaningful activities with clients with mild to moderate dementia. More insight in which tools are appropriate for which frail elderly might support professionals.

Bearing the impact of the social and physical environment in mind, external conditions for self management of frail elderly are not always optimal. Dutch local governments are responsible for community prevention and arrangement of home adaptations. Primary care professionals might benefit from close collaboration with local governments as it increases their possibilities to influence decision-making with regard to self management of their clients.

\subsection{Conclusions and implications}

Disability-prevention in frail elderly is a priority for elderly care. Findings from our reviews point to the development of tailor-made, multifactorial, and multidisciplinary disability prevention programmes in primary care, including elements as screening, self management and case management. We developed an innovative protocol for such a programme. This thesis shows that this programme has potential to address the needs of community-dwelling frail elderly. More specifically, our feasibility study showed that care professionals assessed the programme as acceptable and deliverable, providing them with a more comprehensive view on the older person. Further, the programme supported them to focus on prevention and promoted interdisciplinary cooperation. The older clients were positive about the support they received and a focus on the needs of frail elderly for being engaged in meaningful activities seems a fruitful principle. At the same time it is clear that various elements of our programme need further attention and its effectiveness still has to be demonstrated.

\subsubsection{Implications for practice}

At this point, no golden standard for identification of frail community-dwelling elderly is available yet. As a consequence, instruments such as the Groningen Frailty Indicator should be used with caution in primary care. General practitioners who 
wish to start pro-active elderly care could consider use of a short screening tool, though are recommended to find strategies to reduce the number of false positives. The additional use of clinical judgment after the preliminary screening might be an option. Clinical judgment could be based on a recent contact (Dutch elderly 75 or older see their GP on average 9 times a year ${ }^{27}$ ) or a new appointment in which the GP focuses on recent transitions in functioning.

Screening should be followed by a tailor made, multifactorial intervention strategy for those identified as frail. At this point no definitive recommendations can be made for effective disability-prevention in community-dwelling frail elderly. However, the studies in this thesis do provide useful elements for constructing a programme such as assessments, client centered care, interdisciplinary cooperation and (a toolbox of) interventions.

An intervention involving so many elements is complex. Implementation requires a project-based approach, with attention to mutual understanding of professionals of the programme's philosophy, its models and methods. Professionals in primary care have to discover and to learn together what it means to work interdisciplinary. Understanding that goals of the frail older person are the points of departure for collaboration and not those of the disciplines involved, and understanding how to use meaningful activities as means and ends of treatment are important conditions. Education and guidance is necessary to enhance the learning process and reach successful implementation.

\subsubsection{Implications for research}

Additional research is needed regarding both screening and interventions in community-dwelling frail elderly.

\section{Screening}

Short screening tools such as the Groningen Frailty Indicator and Tilburg Frailty Indicator have potential. Additional research is needed on the efficiency of these tools (can smaller subsets of items render similar results?) and on optimizing prediction by exploring results when items from different tools are combined.

Postal screening with self report measures seems insufficient for effective identification of frail elderly. Future studies should focus on supplementary strategies for identification of frail elderly, such as clinical judgment or the addition of performance based measures to self-report tools. For the latter, research on new technological developments is recommended. 


\section{Intervention programme}

Further research is needed on several parts of the programme:

- It is important to focus future research on fine-tuning of the self management approach (including reflection on performance of activities, goal setting, action planning and follow-up) for community-dwelling frail elderly with complex problems.

- Future research is needed to optimize the efficacy of interdisciplinary collaboration, including the supporting role of technology.

- Technology seems an effective way of supporting frail elderly in performance of activities. More research is necessary to develop and test high and low tech assistive devices for frail older persons with the aim to improve quality of life of frail elderly. 


\section{References}

1. Fried LP, Young Y, Rubin G, Bandeen-Roche K. Self-reported preclinical disability identifies older women with early declines in performance and early disease. J Clin Epidemiol. 2001;54(9):889-901.

2. Ensrud KE, Ewing SK, Taylor BC, Fink HA, Cawthon PM, Stone KL, et al. Comparison of 2 frailty indexes for prediction of falls, disability, fractures, and death in older women. Arch Intern Med. 2008;168(4):382-9.

3. Sarkisian CA, Gruenewald TL, John Boscardin W, Seeman TE. Preliminary evidence for subdimensions of geriatric frailty: the MacArthur study of successful aging. J Am Geriatr Soc. 2008;56(12):2292-7.

4. Karunananthan S, Wolfson C, Bergman H, Beland F, Hogan DB. A multidisciplinary systematic literature review on frailty: overview of the methodology used by the Canadian Initiative on Frailty and Aging. BMC Med Res Methodol. 2009;9:68.

5. Jette AM. Toward a common language for function, disability, and health. Phys Ther. 2006;86(5):726-34.

6. Avlund K. Disability in old age. Longitudinal population-based studies of the disablement process. Dan Med Bull. 2004;51(4):315-49.

7. Van lersel MB, Olde Rikkert MGM. Frailty criteria give heterogeneous results when applied in clinical practice. Letter to the editor. J Am Geriatr Soc 2006;54(4):728-9.

8. Steinbusch C. Screening of frail elderly in the community. The feasibility and psychometric properties of three instruments Maastricht: Maastricht University; 2008.

9. Sourial N, Wolfson C, Bergman H, Zhu B, Karunananthan S, Quail J, et al. A correspondence analysis revealed frailty deficits aggregate and are multidimensional. J Clin Epidemiol. 2009;63(6):647-54.

10. Ravaglia G, Forti P, Lucicesare A, Pisacane N, Rietti E, Patterson C. Development of an easy prognostic score for frailty outcomes in the aged. Age Ageing. 2008;37(2):161-6.

11. Abellan van Kan G, Rolland Y, Houles M, Gillette-Guyonnet S, Soto M, Vellas B. The Assessment of Frailty in Older Adults. Clin Geriatr Med 2010;26:275-86.

12. Gill TM, Allore HG, Gahbauer EA, Murphy TE. Change in disability after hospitalization or restricted activity in older persons. JAMA. 2010;304(17):1919-28.

13. Gill TM, Gahbauer EA, Allore HG, Han L. Transitions between frailty states among community-living older persons. Arch Intern Med. 2006;166(4):418-23.

14. Rockwood K, Song X, MacKnight C, Bergman H, Hogan DB, McDowell I, et al. A global clinical measure of fitness and frailty in elderly people. CMAJ. 2005;173(5):489-95.

15. Syddall H, Cooper C, Martin F, Briggs R, Aihie Sayer A. Is grip strength a useful single marker of frailty? Age Ageing. 2003;32(6):650-6.

16. VanSwearingen JM, Brach JS. Making geriatric assessment work: selecting useful measures. Phys Ther. 2001;81(6):1233-52.

17. Witte de LP, Widdershove G, Kempen GIJM, Neyens JCL, Rossum van E, Schols JM. Monitoring van frailty-indicatoren bij kwetsbare ouderen; ontwikkeling en evaluatie van een ICT-gebaseerd monitoringsysteem ter ondersteuning van zelfmanagement. Maastricht: ZONMW subsidieaanvraag; 2008.

18. Zorginnovatieplatform. Probleemanalyse Innovatie in de zorg. Den Haag: Zorginnovatieplatform; 2008.

19. Bartholomew LK, Parcel GS, Kok G, Gottlieb NH. Planning Health Promotion Programs: Intervention Mapping. San Francisco: Jossey-Bass; 2006.

20. Rogers E. Diffusion in preventive innovations. Addicitive behaviors. 2002;27(6):989-93.

21. Kempen GIJM. Preventie en ouder worden: mogelijkheden voor zelfmanagement. Epidemiologisch bulletin. 2006;41(2):27-32.

22. Bodenheimer T, Grumbach K. Improving primary care. Strategies and tools for better practice. New York: Lange Medical Books/McGraw-Hill; 2007.

23. Dossa A, Capitman JA. Community-based disability prevention programs for elders: predictors of program completion. J Gerontol Soc Work. 2010;53(3):235-50. 
24. Elzen $H$, Slaets JP, Snijders TA, Steverink N. Do older patients who refuse to participate in a selfmanagement intervention in the Netherlands differ from older patients who agree to participate? Aging Clin Exp Res. 2008;20(3):266-71.

25. CBOG. Nieuwe uitdagingen voor zorgverleners in de eerstelijn: Eerstelijnszorg in CBOG -perspectief Utrecht: College voor de Beroepen en Opleidingen in de Gezondheidszorg; 2010.

26. Graff MJ, Vernooij-Dassen MJ, Thijssen M, Dekker J, Hoefnagels WH, Rikkert MG. Community based occupational therapy for patients with dementia and their care givers: randomised controlled trial. BMJ. 2006;333(7580):1196.

27. CBS.Door de huisarts geregistreerde contacten [database on the Internet]. Centraal Bureau voor de statistiek. 2010 . Available from: http://www.cbs.nl.

28. Metzelthin SF, van Rossum E, de Witte LP, Hendriks MR, Kempen GI. The reduction of disability in community-dwelling frail older people: design of a two-arm cluster randomized controlled trial. BMC Public Health. 2010;10(1):511. 

Summary 
The population is ageing in Western countries. Balancing the costs and benefits of healthcare will be a key aim for these countries and older people need to be supported to remain healthy and independent for as long as possible. Therefore, a strategic shift in elderly care towards prevention and early intervention is necessary. A group at high risk for developing adverse outcomes as disability, mortality, and institutionalisation are frail older people. Frailty refers to an increased vulnerability to stressors due to impairments in multiple, inter-related systems that lead to a decline in homeostatic reserve and resiliency. Despite a lack of consensus about the definition of frailty, there seems to be agreement on the clinical usefulness of the concept.

Disability is defined as experienced difficulty in performing activities in any domain of life. For (frail) older people, difficulties in performing activities feature among the most important causes of a reduced quality of life. Recent Dutch policy reports put a major focus on development of interventions that focus on promoting independent functioning in daily life for (vulnerable) older persons with an important role for primary care, screening of vulnerable groups, case management and multidisciplinary cooperation.

Community-based intervention research in the field of frail elderly is relatively new. Studies so far show conflicting findings and gaps in knowledge. Key issues are still unclear: How to define frailty, how to identify community-dwelling frail elderly and what interventions are effective in preventing disability?

The main objective of this thesis was to develop a disability-prevention programme for community-dwelling frail elderly. To achieve this, several issues related to the concept of frailty, identification of frail elderly and the approach and organization of disability prevention were addressed. The research questions for this thesis were:

What is the state of art in the frailty literature concerning the concept of frailty and instruments for identifying frail elderly?

What is the validity of postal screening questionnaires to identify communitydwelling frail elderly?

What is known in the literature about effective elements of disability-prevention programmes and how can they be integrated into a comprehensive and coherent programme?

To what extent is a new interdisciplinary disability-prevention programme for community-dwelling frail elderly feasible in Dutch primary care?

Chapter 1 provides an introduction into the topic under research. We describe the consequences of ageing, the prevalence estimates of frailty and the necessity for 
effective interventions to prevent disability. Further, we present the main objective for this thesis and the research questions.

Chapter 2 presents an overview of the literature related to the concept of frailty, screening instruments and interventions for community-dwelling frail elderly. No consensus yet exists on the definition of frailty. The debate has focused on whether frailty should be defined purely in terms of biomedical factors (addressed to as physical frailty) or whether other factors should be included as well. Related to it, the debate continues on appropriate indicators for frail older people and how to assess these. As a consequence, no golden standard is present for identifying frail elderly. Overviews of community-based interventions are scarce and face difficulties due to a lack of consensus for an explicit conceptual frailty definition and validated practical screening criteria.

In Chapter $\mathbf{3}$ we describe the evaluation and comparison of the internal consistency and construct validity of three instruments to detect frail elderly, using a postal screening procedure: the Groningen Frailty Indicator (GFI), the Tilburg Frailty Indicator (TFI) and the Sherbrooke Postal Questionnaire (SPQ). A questionnaire was sent to nearly 700 community-dwelling older people ( $\geq 70$ years). The response rate was $77 \%$. Prevalence estimates of frailty ranged from $40 \%$ to $59 \%$. The GFI and TFI showed high internal consistency and construct validity in contrast to the SPQ.

Chapter 4 focuses on the predictive validity of the same instruments for development of disabilities, mortality and hospital admissions over one year follow-up $(n=430)$. Adjusted odds ratios showed that those identified as frail by any of the three instruments have more than twice the risk for developing disabilities compared to the non-frail group; those identified as frail by the TFI and SPQ have more than twice the risk of being admitted to a hospital. Diagnostic values of the three instruments are, however, insufficient. Sensitivity and specificity for development of disabilities e.g., are between 60 and $80 \%$ and between 50 and $70 \%$ respectively. In a two-step screening approach, these instruments would select a large number of false positives, implying that many older persons would have to go through an unnecessary additional extensive assessment.

In chapter $\mathbf{5}$ we present a systematic review into the content, methodological quality and effectiveness of intervention studies for the prevention of disability in community-dwelling physically frail elderly. We found no evidence for the effect of nutritional interventions on disability outcomes, nor for the effect of single lower extremity strength training. However, we found indications that relatively long-lasting and high-intensive multicomponent exercise programs have a positive effect on ADL and IADL disability for community-living moderate physically frail older persons. 
Chapter 6 presents a second review on interventions targeted at communitydwelling frail older persons (not only physical frail) providing insight in promising elements of 49 interventions. This narrative review showed that future interventions may be directed towards tailor-made, multidisciplinary and multifactorial interventions, with individualised assessment and interventions conducted by a (primary) care team, involving case management and long-term follow-up. These tailor-made programmes may include a physical exercise component for moderate physically frail older persons and a technology component as well.

In Chapter 7 we describe the content of the interdisciplinary disability prevention programme as developed by our research team and a multidisciplinary professional task group. The programme aims to support frail older persons in performance of activities meaningful to them by targeting risk factors and problems in performing these activities. The general practitioner (GP) and practice nurse (PN) comprise the core team that can be extended by other professionals such as the occupationaland physical therapist. The programme includes six steps: (1) screening, (2) assessment, (3) analysis and preliminary action plan, (4) agreement on action plan, (5) execution of the action plan (toolbox parts), and (6) evaluation and follow-up. Screening and individual assessment, tailor-made and client-centred care, selfmanagement support, case management and interdisciplinary cooperation are important principles of the programme. The programme includes a toolbox of five interventions that can be utilized in a flexible manner.

Chapter 8 presents the results of the feasibility study of our programme. Forty-one older persons and ten professionals took part in this study that monitored the actual use of the disability prevention programme and experiences of frail elderly and professionals. Most parts of the protocol were followed. Parts of self management support (face to face meetings to discuss the action plan, the arrangement of follow-up and the use of motivational interviewing techniques) were often not applied according to protocol. Deviations and non-use of protocol parts were related to time constraints and difficulties in executing specific elements of the protocol in practice. The participants were very positive about the support they received; they appreciated the personal attention and the support for obtaining personal goals.

Professionals regarded the programme as acceptable and deliverable for those experiencing problems in ADL and/or IADL. They stated that the programme led to a more comprehensive view on the older person as a consequence of the multifactorial perspective on frailty and the focus on (meaningful) activities. Furthermore, professionals expressed that the protocol, guiding both process and content, clarified each person's role (e.g., the PN as case manager) and enhanced interdisciplinary cooperation. A substantial proportion of the selected elderly were pre-frail, 
according to the GP and PN. We concluded that professionals involved regarded the interdisciplinary disability-prevention programme as feasible for Dutch primary care. Several of its elements (e.g. screening and self management), though, need further attention.

Finally, chapter 9 provides an overview of the main findings of our thesis, followed by methodological and theoretical considerations. This chapter 9 ends with conclusions and implications for practice and research with respect to screening and intervention.

Disability prevention in frail elderly is a priority for elderly care. We developed an innovative programme. This thesis shows that this programme has potential to address the needs of community-dwelling frail elderly. More specifically, our feasibility study showed that care professionals assessed the programme as acceptable and deliverable, providing them with a more comprehensive view on the older person. Further, the programme supported them to focus on prevention and promoted interdisciplinary cooperation. The older clients were positive about the support they received and a focus on the needs of frail elderly for being engaged in meaningful activities seems a fruitful principle. However, an intervention involving so many elements is complex. Implementation requires a project-based approach, in which professionals learn to work interdisciplinary, to support self management and to use meaningful activities as means and ends of treatment.

The feasibility study also clarified that the self management approach and the postal screening procedure need reconsideration. More pragmatic tools for professionals to support self management are necessary. Postal screening questionnaires based on a multifactorial approach to frailty do have potential, but their diagnostic values are low. More research is required to optimize the predictive validity of frailty screening tools. 

Samenvatting 
De vergrijzing van de bevolking stelt westerse landen voor uitdagingen. Het balanceren tussen de kosten en opbrengsten van de ouderenzorg krijgt een hoge prioriteit en een belangrijke vraag is hoe ouderen ondersteund kunnen worden om zolang mogelijk gezond en onafhankelijk te blijven. Een strategische verschuiving in de ouderenzorg naar preventie en vroegtijdige interventies is noodzakelijk. Speciale aandacht is daarbij nodig voor kwetsbare zelfstandig wonende ouderen, die de komende jaren sterk in aantal zullen toenemen. Deze ouderen hebben een groter risico op ongewenste uitkomsten zoals beperkingen in activiteiten, ziekenhuisopnames, vallen en mortaliteit. Kwetsbaarheid refereert aan een toegenomen gevoeligheid voor stressoren als gevolg van verstoringen in meerdere, onderling gerelateerde systemen, die resulteren in afname van reservecapaciteit. Ondanks een gemis aan consensus over de precieze definitie van kwetsbaarheid, lijkt er over de bruikbaarheid van het concept in de praktijk overeenstemming te zijn.

Beperkingen in activiteiten zoals zelfverzorgingsactiviteiten, sociale activiteiten of hobby's hebben een grote invloed op de kwaliteit van leven van ouderen. De Nederlandse overheid ziet het als een speerpunt om (kwetsbare) ouderen te ondersteunen om onafhankelijk te zijn en te kunnen participeren in de maatschappij. Diverse beleidsrapporten benadrukken de ontwikkeling van interventies gericht op het zelfstandig functioneren van ouderen, met een belangrijke rol voor de eerste lijn, voor screening, case management en multidisciplinaire samenwerking. Onderzoek naar eerstelijns interventies voor kwetsbare ouderen is relatief nieuw. Tot nu toe laten effectstudies geen eenduidige resultaten zien en er zijn nog veel hiaten in kennis: Hoe moet kwetsbaarheid gedefinieerd worden, hoe kunnen kwetsbare ouderen opgespoord worden en welke interventies zijn effectief in het voorkomen van beperkingen in activiteiten?

Het hoofddoel van dit proefschrift is het ontwikkelen van een programma voor thuiswonende kwetsbare ouderen, gericht op het voorkomen van beperkingen in activiteiten. Om dit te bereiken werden diverse zaken rond het concept kwetsbaarheid, de identificatie van kwetsbare ouderen en de aanpak en organisatie van preventie van beperkingen uitgezocht. De onderzoeksvragen voor dit proefschrift zijn:

- Wat is in de literatuur bekend over het concept kwetsbaarheid en instrumenten om kwetsbare ouderen te identificeren?

- Wat is de validiteit van per post verstuurde screeninginstrumenten voor het identificeren van thuiswonende kwetsbare ouderen?

- Wat is in de literatuur bekend over effectieve elementen van programma's voor kwetsbare ouderen gericht op beperkingen in activiteiten en hoe kunnen deze samengebracht worden in een innovatief en integraal programma?

- In welke mate is dit nieuwe interdisciplinaire programma voor thuiswonende kwetsbare ouderen ter voorkoming van beperkingen in activiteiten hanteerbaar in de Nederlandse eerstelijnszorg? 
Hoofdstuk 1 geeft een introductie op het thema van het onderzoek. We beschrijven de consequenties van de vergrijzing, de prevalentie van kwetsbaarheid in Nederland en de urgentie voor het ontwikkelen van effectieve interventies die beperkingen in activiteiten kunnen voorkomen. Verder presenteren we het hoofddoel van het proefschrift en de onderzoeksvragen.

Hoofdstuk 2 presenteert de stand van zaken in de literatuur over het concept kwetsbaarheid en screeninginstrumenten voor het opsporen van thuiswonende kwetsbare ouderen. Er is geen consensus over de definitie van kwetsbaarheid. De discussie in de literatuur gaat vooral over de vraag of kwetsbaarheid gedefinieerd moet worden vanuit een biomedisch perspectief (ook wel fysieke kwetsbaarheid genoemd) of dat ook andere dan fysieke factoren onderdeel van de definitie moeten zijn (multifactorieel perspectief). Hieraan gerelateerd is er debat over de vraag welke indicatoren voor kwetsbaarheid de juiste zijn en welke wijze van opsporen effectief is. Een gouden standaard voor het opsporen van kwetsbare ouderen is nog niet aanwezig. Overzichten van interventies in de $1^{\mathrm{e}}$ lijn zijn schaars als gevolg van het gemis aan een consensus over de definitie van kwetsbaarheid en gevalideerde criteria om de juiste ouderen te selecteren.

In hoofdstuk 3 rapporteren we over de interne consistentie en construct validiteit van drie (zelfrapportage) screeninginstrumenten die per post verstuurd kunnen worden, met het doel om kwetsbare ouderen op te sporen. In dit onderzoek werden de Groningen Frailty Indicator (GFI), de Tilburg Frailty Indicator (TFI) en de Sherbrooke Postal Questionnaire (SPQ) naar bijna 700 ouderen ( $\geq 70$ jaar) gestuurd. De respons bedroeg $77 \%$. Prevalentieschattingen van kwetsbaarheid varieerden van $40 \%$ tot $59 \%$. De GFI en TFI lieten zowel een hoge interne consistentie als construct validiteit zien, in tegenstelling tot de SPQ.

Hoofdstuk 4 richt zich op de predictieve validiteit van dezelfde screeninginstrumenten voor het ontwikkelen van beperkingen, ziekenhuisopname en mortaliteit, na 1 jaar follow-up ( $n=430)$. De door elk van de drie instrumenten als kwetsbaar geïdentificeerde ouderen hadden een tweemaal zo grote kans om beperkingen in activiteiten te ontwikkelen; ouderen geïdentificeerd als kwetsbaar door de TFI en SPQ hadden ook een tweemaal zo grote kans op één of meerdere ziekenhuisopnamen. De diagnostische waarden van de instrumenten zijn echter niet toereikend. Sensitiviteit en specificiteit voor ontwikkeling van beperkingen in activiteiten zijn respectievelijk tussen de 60 en $80 \%$ en tussen de 50 en $70 \%$. De instrumenten leidden tot teveel fout-positieve testuitslagen; zonder verdere aanpassingen zouden er in een tweetraps-screeningprocedure relatief veel ouderen onnodig een aansluitend uitgebreid assessement moeten ondergaan. 
In hoofdstuk 5 presenteren we een systematische literatuurstudie naar de inhoud, de methodologische kwaliteit en de effectiviteit van interventiestudies gericht op beperkingen in activiteiten bij thuiswonende fysiek kwetsbare ouderen. Er werd geen bewijs gevonden voor het effect van voedingsinterventies en het effect van krachttraining van de onderste extremiteiten op beperkingen in activiteiten. $\mathrm{Er}$ is enige indicatie dat langdurende hoogintensieve multicomponent beweegprogramma's een effect hebben op ADL en IADL beperkingen in activiteiten bij mild kwetsbare ouderen.

Hoofdstuk 6 rapporteert over een tweede literatuuronderzoek naar interventies voor thuiswonende kwetsbare ouderen (niet alleen gericht op fysieke kwetsbaarheid), bedoeld om veelbelovende elementen te identificeren. Dit narratieve literatuuronderzoek concludeerde dat toekomstige interventies zich zouden kunnen richten op een multidisciplinaire en multifactoriële aanpak op maat, met assessments en interventies uitgevoerd door één (eerstelijns) team met case management en lange termijn follow-up. Deze programma's zouden een bewegingscomponent (voor milde kwetsbare ouderen) en het gebruik van ondersteunende technologie kunnen bevatten.

In hoofdstuk 7 beschrijven we de inhoud van het interdisciplinaire programma ter voorkoming van beperkingen in activiteiten, zoals door ons ontwikkeld in nauwe samenwerking met een multidisciplinaire projectgroep van zorgprofessionals. Het hoofddoel is het ondersteunen van kwetsbare ouderen bij het (blijven) uitvoeren van betekenisvolle activiteiten, door de aanpak van risicofactoren en huidige problemen in het uitvoeren van deze activiteiten. De huisarts en praktijkondersteuner vormen het kernteam, dat aangevuld kan worden met andere professionals zoals de ergotherapeut en fysiotherapeut. Er zijn zes stappen: (1) screening, (2) assessment, (3) analyse, probleemlijst en voorlopig plan van aanpak, (4) vaststellen van plan van aanpak, (5) uitvoering van dit plan (toolbox onderdelen), en (6) evaluatie en followup. Screening en individuele assessments, maatwerk en cliëntgerichte zorg, zelfmanagement ondersteuning, case management en interdisciplinair samenwerken zijn belangrijke uitgangspunten in het aanbieden van het programma. Het programma bevat een toolbox met vijf verschillende interventies die flexibel inzetbaar zijn.

Hoofdstuk 8 presenteert de resultaten van het onderzoek naar de hanteerbaarheid van ons interventieprogramma. Eenenveertig kwetsbare ouderen en 10 professionals participeerden in deze studie die het daadwerkelijk gebruik van het programma en de ervaringen van ouderen en professionals in kaart bracht. De meeste onderdelen van het programma werden in praktijk gebracht. Echter, belangrijke onderdelen van de zelfmanagement ondersteuning werden vaak niet volgens protocol uitge- 
voerd. Oorzaken van afwijkingen van of het niet gebruiken van het protocol werden toegeschreven aan tijdsaspecten of problemen met de toepassing van de onderdelen.

De ouderen waren zeer positief over het programma; zij waardeerden de persoonlijke aandacht en de ondersteuning die zij ontvingen voor het bereiken van persoonlijke doelen. De professionals zijn van mening dat het programma geschikt is voor kwetsbare ouderen die al problemen in ADL of IADL ervaren. Zij verklaarden dat het programma tot een breder beeld van de oudere leidt als gevolg van het multifactoriële perspectief op kwetsbaarheid en de nadruk op betekenisvolle activiteiten. Verder gaven zij aan dat het protocol, als richtlijn voor processen en inhoud, verduidelijking bood over de rollen van de disciplines en interdisciplinaire samenwerking stimuleerde. Professionals beschouwen het programma als hanteerbaar voor toepassing in de eerste lijn. Echter, een deel van deze ouderen was in de ogen van de huisarts en praktijkondersteuner niet of slechts mild kwetsbaar. Wij concludeerden dat het programma potentie heeft; maar er zijn nog een aantal onderdelen (zoals screening en zelfmanagement) waarvoor verdere aandacht nodig is.

Tot slot geeft hoofdstuk 9 een samenvatting van de belangrijkste resultaten van de verschillende deelstudies, gevolgd door een methodologische en theoretische beschouwing. We eindigen dit hoofdstuk met implicaties voor de praktijk en verder onderzoek aangaande screening en interventie.

Het voorkomen van beperkingen in activiteiten is een prioriteit in de ouderenzorg. We ontwikkelden een innovatief programma dat tegemoet komt aan de behoeften van thuiswonende kwetsbare ouderen aan onafhankelijkheid en participatie. De betrokken zorgprofessionals vonden het programma acceptabel en uitvoerbaar. Het programma ondersteunt hen bij het leveren van preventieve zorg, geeft een veel completer beeld van de oudere en stimuleert interdisciplinaire samenwerking. De ouderen waren positief over de ondersteuning van de professionals. De focus van het programma op de betekenisvolle activiteiten van kwetsbare ouderen lijkt een zeer waardevol uitgangspunt.

Echter, een programma met zoveel verschillende elementen is complex. De implementatie in de praktijk zal een projectmatige aanpak vereisen, waarin professionals leren om interdisciplinair samen te werken, zelfmanagement te ondersteunen en betekenisvolle activiteiten te gebruiken als middel en als doel.

De hanteerbaarheidsstudie liet zien dat de ondersteuning van zelfmanagement en de procedure voor screening opnieuw bekeken moeten worden. Meer praktische tools voor professionals om de ondersteuning van zelfmanagement vorm te geven zijn nodig. Screeninginstrumenten gebaseerd op een multifactorieel perspectief van 
kwetsbaarheid hebben potentie, maar de diagnostische waarden zijn nog niet toereikend. Aanvullend onderzoek naar de predictieve validiteit is derhalve nodig. 
Appendix 


\section{The Groningen Frailty Indicator (GFI)}

\section{Physical components}

Are you able to carry out these tasks single-handedly and without any help? (The use of help resources such as a walking stick, walking frame or wheelchair is considered to be independent.)

1. Shopping

2. Walking around outside (around the house or to the neighbours)

3. Dressing and undressing

4. Going to the toilet

5. What mark do you give yourself for physical fitness? (scale 0 to 10)

6. Do you experience problems in daily life due to poor vision?

7. Do you experience problems in daily life due to being hard of hearing?

8. During the last 6 months have you lost a lot of weight unwillingly?

( $3 \mathrm{~kg}$ in 1 month or $6 \mathrm{~kg}$ in 2 months)

9. Do you take 4 or more different types of medicine?

\section{Cognitive component}

10. Do you have any complaints about your memory?

\section{Social component}

11. Do you sometimes experience emptiness around yourself?

12. Do you sometimes miss people around yourself?

13. Do you sometimes feel abandoned?

Psychological component

14. Have you recently felt downhearted or sad?

15. Have you recently felt nervous or anxious?

\section{Scoring:}

Questions 1-4: Yes = 0; No = 1

Question 5: 0-6 = 1; 7-10 = 0

Questions 6-9: No = 0; Yes $=1$

Question 10: No = 0; Sometimes = 0; Yes $=1$

Questions 11-15: No = 0; Sometimes = 1; Yes = 1 


\section{The Tilburg Frailty Indicator (TFI) (2nd subscale)}

\section{Physical component}

1. Do you feel healthy?

2. Have you lost a lot of weight recently without wishing to do so?

('A lot' is $6 \mathrm{~kg}$ or more during the last six months or $3 \mathrm{~kg}$ or more during the last month.)

Do you experience problems in your daily life due to:

3. Difficulty in walking?

4. Difficulty with maintaining your balance?

5. Poor hearing?

6. Poor vision?

7. Lack of strength in your hands?

8. Physical tiredness?

\section{Psychological component}

9. Do you have problems with your memory?

10. Have you felt down during the last month?

11. Have you felt nervous or anxious during the last month?

12. Are you able to cope with problems well?

Social component

13. Do you live alone?

14. Do you sometimes wish you had more people around you?

15. Do you receive enough support from other people?

\section{Scoring:}

Question 1: Yes = 0; No = 1

Questions 2-8: No = 0; Yes = 1

Question 9: No = 0; Sometimes $=0 ;$ Yes $=1$

Questions 10-11: No = 0; Sometimes = 1; Yes = 1

Question 12: No $=1$; Yes $=0$

Question 13: No = 0; Yes $=1$

Question 14: No $=0$; Sometimes $=1 ;$ Yes $=1$

Question 15: Yes = 0; No $=1$ 


\section{The Sherbrooke Postal Questionnaire (SPQ)}

1. Do you live alone?

2. Do you take more than three different medications every day?

3. Do you regularly use a cane, a walker or a wheelchair to move about?

4. Do you see well?

5. Do you hear well?

6. Do you have problems with your memory?

Scoring:

Question 1: Yes = 0; No = 1

Questions 2-3: No = 0; Yes = 1

Questions 4-5: Yes = 0; No = 1

Question 6: No = 0; Yes = 1 


\section{Steps of the disability prevention programme}

Screening: GP practice sends GFI to older persons $(\geq 70)$. GFI-score $>5$ : PN calls for home visit

Assessment: PN conducts assessment with older person (and informal caregiver) followed by meeting with GP about necessity for:

- Assessment GP

- Assessment OT and/or PT (advised in case of concerns or problems in performing activities)

- Additional assessments

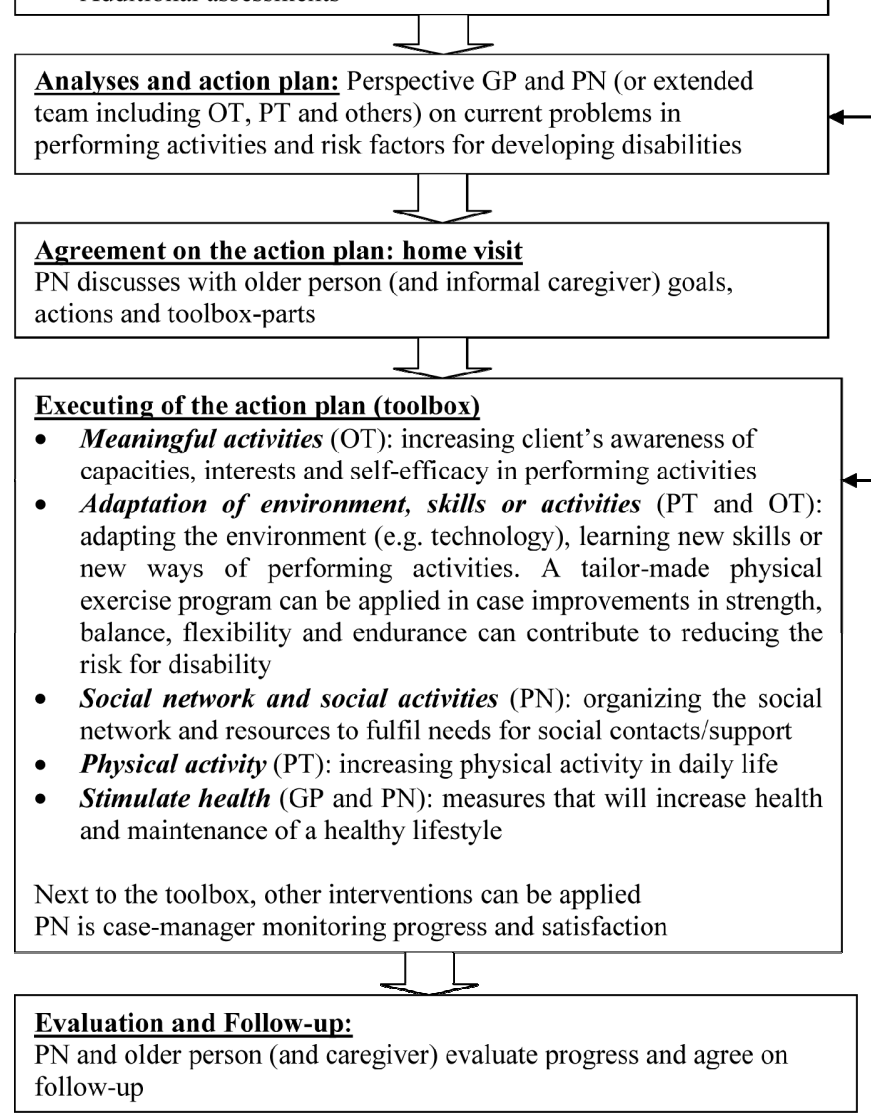

Multidisciplinary guidelines on geriatric problems:

- Dementia

- Delirium

- Polypharmacy

- Fall prevention

- Constipation and fecal incontinence

- Urine incontinence

- Depression

- Malnutrition/ dehydration

Standards of Dutch College of General

Practitioners e.g.:

- Diabetes

- COPD

- Cardiovascular risk management

- Arthritis 



\section{Dankwoord}


Dit promotieonderzoek was een leerproces en een tocht die ik met bijzondere mensen mocht maken. Volgens de flow theorie kom je in de prettige toestand van flow als je je volledig geeft in de uitvoering van uitdagende betekenisvolle activiteiten. Ik heb de afgelopen jaren veel flow ervaren, al gaat het te ver om een promotieonderzoek een voortdurende staat van flow te noemen. Er waren momenten dat het allemaal wat minder vlotte. Desondanks beschouw ik mijn promotieonderzoek als een cadeautje: zaken bestuderen, een bijdrage kunnen leveren aan kennisontwikkeling en samenwerken met bevlogen mensen. Ik ben oprecht dankbaar dat ik deze kans heb gekregen.

Mijn eerste dank gaat uit naar mijn promotieteam: Wim van den Heuvel en Luc de Witte (promotores) en Erik van Rossum (co-promotor). Samen hebben we inhoud gegeven aan een aanvankelijk summier plan voor een promotieonderzoek rondom kwetsbare ouderen. Jullie zijn mijn gidsen geweest in deze jaren. Jullie paren grote kennis aan een enorme ervaring in onderzoek en beleid en een behoorlijke dosis nononsense. Uiteindelijk staan jullie altijd voor hetgeen het allemaal om te doen is: verbetering van de zorg voor cliënten. Luc en Wim, jullie hebben uitstekend de grote lijnen van het project bewaakt. Overleg met jullie levert telkens weer nieuwe inzichten op. Jullie overzicht en kritisch vermogen leidden tot vragen die 'ook' gesteld moesten worden. Erik, epidemioloog met humor (combinatie schijnt weinig voor te komen), eeuwig bedankt voor de vele feedback, het geduld, het gezoek, de wijze raad, de grapjes, de vriendschap. Ik heb veel respect voor de wijze waarop je mensen weet te binden door hen vertrouwen en goede raad te geven.

Veel dank zijn we verschuldigd aan alle ouderen die bereid waren te participeren in de verschillende deelstudies. Zonder hun bereidwilligheid zou dit proefschrift er niet liggen. Gedurende het promotietraject zijn er veel mensen geweest waarmee we zeer prettig samengewerkt hebben. Silke Metzelthin, als ('mijn') voormalige ergotherapie-student heb je ons voortreffelijk ondersteund tijdens je masterstudie aan de Universiteit Maastricht. Inmiddels ben je promovenda en evalueer je ons Zorg uit Voorzorg programma. Herbert Habets, Walther Sipers en Sjoerd Hobma, ik heb enorme waardering voor jullie kennis en daadkracht om veldprojecten van de grond te krijgen. Veel dank ook aan alle mensen die hun grote expertise in hebben gezet voor het ontwikkelen en testen van Zorg uit Voorzorg; Henk Jochems, Inge Systermans, Margot van Melick, Frank Vlaskamp, Rob de Ruijter, Frederique Prompers, Michel Oostdam, Vienna Denis, Simone Denis, Marcel Jacobs, Barry Nugteren, Anniek Nilwik, Marijn van Oord, Marlou Wolters en Lilo Crasborn. Ook veel dank aan de huisartsenpraktijken die meewerkten aan de studies naar de screeningsinstrumenten; Dokters bij Jansen (Rijk en Snijder in Amersfoort), A.C.J.Matthee (Roermond) en Hundscheid (Roggel). Ook veel dank aan Claudia Steinbusch voor haar pilot naar schriftelijke screening op kwetsbaarheid, aan Marieke Spreeuwenberg en 
Melanie Kleynen voor statistische adviezen, en Ruud Kempen voor zijn waardevolle bijdrages aan verschillende studies.

Zeer belangrijk is de facilitering van het promotieonderzoek. Luc de Witte en Frits Benjamins maakten aanvankelijk de start mogelijk vanuit de kenniskring Autonomie en Participatie en de Faculteit Gezondheid en Zorg. Ook aan het College van Bestuur van de Hogeschool Zuyd ben ik bijzonder veel dank verschuldigd voor de financiële bijdragen aan het promotietraject en de getoonde interesse. Sandra Beurskens volgde Luc in 2007 op als lector van de kenniskring Autonomie en Participatie. Sandra, geweldig hoe je altijd geïnteresseerd bent, meedenkt en op zoek gaat naar oplossingen. Collega's van de kenniskringen Autonomie en Participatie en Technologie in de Zorg, mijn dank voor jullie betrokkenheid. Susy, Ruth en Jessie, dank voor het delen van ervaringen en jullie adviezen. Ik wil hier vooral ook mijn dank uitspreken aan mijn collega's van de opleiding ergotherapie. Het is prettig vertoeven in zo'n hecht en innovatief team. Jullie hebben in deze jaren altijd veel belangstelling getoond voor mijn promotieonderzoek en laten blijken dat jullie het belangrijk vinden. Dat is voor mij van grote waarde geweest.

Er kwam tijdens het promotietraject nog een warm nest bij: de vakgroep Verpleging en Verzorging van Universiteit Maastricht. Ik was daar vooral de eerste jaren wekelijks aanwezig. Heerlijke rustige werkplek! Ruud Kempen en Jan Hamers, dank voor de kans om me bij jullie groep te voegen. Verder ook dank aan alle medewerkers van de vakgroep voor de gezelligheid en de bereidheid tot delen van ervaringen en kennis.

Heren van de poolclub, mijn dank voor het broodnodige geouwehoer dat het leven zo relativeert. Over het niveau van het spel zouden we het verder niet hebben. Metka en de cursisten van de Sloveense les, dank voor jullie belangstelling en de berusting dat er in de afgelopen jaren weinig progressie in mijn Sloveense taalgebruik zat. Meghan, thank you for correcting my English and the picture of me on this thesis. Nico, geweldig dat jij (met Silke) paranimf wilt zijn.

Rad bi se zahvalil moji slovenski družini, tastu, tašči in svaku. $V$ tem času smo preživeli kar nekaj dopustov na Dobravici, ampak od mene niste imeli prav veliko, ker sem vedno delal na računalniku. Vaša topla in okusna skrb je omogočila, da sva z Barbaro imela proste roke za delo, z vednostjo, da se Nika zabava in preživlja čas v oličnih rokah. Hvala lepa, Jože, Frančiška in Boštjan.

Anton en Joke, mijn ouders, Muriël, mijn zus, ik dank jullie voor de onvoorwaardelijke steun en de goede zorgen, niet alleen in deze periode van promotie. We zijn allemaal trots op elkaar. 
Barbara Piškur, zonder jouw vertrouwen in mij was ik nimmer in dit promotieonderzoek gestapt. De nieuwe rollen van ouder en promovendus vergden de nodige aanpassingen. Ondertussen ben jij ook met een promotieonderzoek gestart. Ik ben je enorm dankbaar voor je steun en begrip, en de ruimte die je me gaf. Ik ben enorm trots op de wijze waarop we het samen rooien. Uiteraard is dat met onze fantastische dochter ook een eitje. Nika, lieffie, zonnetje in huis, papa komt zo spelen. Nee, echt waar, alleen nog even dit dankwoord afmaken. Dank je wel, allemaal. 
Curriculum vitae 
Ramon Daniëls werd op 4 mei 1964 geboren in Oostzaan. In 1983 behaalde hij het VWO-diploma aan de Rijksscholengemeenschap te Harderwijk. Aansluitend volgde hij de opleiding ergotherapie in Weesp, die hij in 1987 afsloot. Direct hierna startte hij met Nico Oskam een samenwerking in organisatie van symposia over CVArevalidatie en werkte als waarnemend ergotherapeut bij revalidatiecentrum Heliomare. Begin 1989 trad Ramon in dienst als ergotherapeut bij revalidatiecentrum Amsterdam. Hier was hij vooral betrokken bij de revalidatie van CVA patiënten en coaching van ergotherapeuten in de advisering van voorzieningen. In 1992 stapte hij over naar het Nederlands Instituut voor Zorg en Welzijn (nu Vilans) voor een project over signalering en advisering van hulpmiddelen en woningaanpassing door wijkverpleegkundigen. Na dit project was Ramon wederom als ergotherapeut voor revalidatiecentrum Amsterdam werkzaam en in 1994 startte hij met de HGZO-opleiding tot paramedisch docent aan de Vrije Universiteit te Amsterdam. De stage tijdens deze opleiding bij de Hogeschool van Amsterdam leidde in 1995 opnieuw tot een overstap van de praktijk naar een organisatie voor kennisontwikkeling en -deling.

Van 1995 tot 2004 was Ramon als docent verbonden aan de opleiding ergotherapie van de Hogeschool van Amsterdam. Deelname aan het Europese congres voor ergotherapie te Madrid in 1996 betekende de start van een internationale oriëntatie. Diverse internationale projecten binnen ENOTHE (European Network of Occupational Therapy in Higher Education) volgden en hij was jarenlang international coördinator. In 2001 rondde hij bovendien de European Masters of Science in Occupational Therapy af. Eind 2003 stapte Ramon over naar Hogeschool Zuyd te Heerlen. Daar combineerde hij onderwijs en coördinatie van onderwijs met managementtaken in het kernteam en een aanstelling bij de kenniskring Autonomie en Participatie van Chronisch zieken. Vanuit deze kenniskring begon hij in 2006 een parttime promotietraject. Onderwijs, management en promotieonderzoek werden door Ramon nog aangevuld met diverse andere projecten, zoals de ontwikkeling van het Gezond Actief Ouder Worden programma en een project rond de marketing van ergotherapie in Parkstad Limburg. Hij is redactielid van het Wetenschappelijk Tijdschrift voor Ergotherapie en lid van de programmacommissie van congressen van Ergotherapie Nederland. Op dit moment is Ramon vanuit het kernteam van de opleiding ergotherapie verantwoordelijk voor de ontwikkeling van het onderzoeksbeleid van de opleiding, is lid van de kenniskring Autonomie en Participatie van Chronisch zieken, en drukdoende nieuwe projectvoorstellen te schrijven rond het thema kwetsbare ouderen en zelfmanagement in de eerste lijn. Ramon is getrouwd met Barbara Piškur. Samen hebben ze een dochter (Nika, 2005). 


\section{Publications}

Metzelthin S, Daniëls R, van Rossum E, de Witte LP, van den Heuvel W, Kempen GIJM. Psychometrische eigenschappen van drie screeningsinstrumenten voor kwetsbaarheid bij thuiswonende ouderen. Tijdschrift voor Gerontologie en Geriatrie. In press.

Daniëls R, van Rossum E, Metzelthin S, Sipers W, Habets $H$, Hobma S, van den Heuvel $W$, de Witte LP. A disability prevention programme for community-dwelling frail older persons. Clinical Rehabilitation. In press.

Daniëls R, Metzelthin S, van Rossum E, de Witte L, van den Heuvel W. Interventies ter voorkoming van beperkingen bij thuiswonende kwetsbare ouderen: een overzicht. Wetenschappelijk Tijdschrift voor Ergotherapie. 2010;4:2-7.

Daniëls R, Metzelthin S, van Rossum E, de Witte L, van den Heuvel W. Interventions to prevent disability in frail community-dwelling older people: an overview. European Journal of Ageing. 2010;7:37-55.

Metzelthin S, Daniëls R, van Rossum E, de Witte L, van den Heuvel W, Kempen GIJM. The psychometric proportions of three self-report screening instruments for identifying frail older people in the community. BMC Public Health. 2010; 10:176.

Daniëls R, Metzelthin S, van Rossum E, de Witte L, Kempen GIJM, van den Heuvel W. Interventies ter voorkoming van beperkingen bij thuiswonende kwetsbare ouderen. Tijdschrift voor Ouderengeneeskunde. 2010; 36:12-16.

Daniëls R, Rossum E van, Witte L de, Kempen GIJM, Heuvel W van. Interventions to prevent disability in frail community-dwelling elderly. BMC Health Services Research. 2008; 8:278.

Daniëls R, van Rossum E, de Witte L, van den Heuvel W. Frailty in older age: Concepts and relevance for occupational and physical therapists. Physical and Occupational Therapy in Geriatrics. 2008;27(2): 81-95.

Daniëls R, van Overbeek R. Zelfmanagement bij ouderen. In: van 't Leven N, van Herwaarde H. Paramedische zorgverlening aan ouderen. Den Haag: Lemma; 2008. 